\title{
The first view of $\delta$ Scuti and $\gamma$ Doradus stars with the TESS mission
}

\author{
V. Antoci, ${ }^{1} *$ M. S. Cunha ${ }^{2}$, D. M. Bowman ${ }^{3}$, S. J. Murphy ${ }^{4,1}$, D. W. Kurtz ${ }^{5}$,
}

T. R. Bedding ${ }^{4,1}$, C. C. Borre ${ }^{1}$, S. Christophe ${ }^{6}$, J. Daszyńska-Daszkiewicz ${ }^{7}$,

L. Fox-Machado ${ }^{8}$, A. García Hernández ${ }^{9,10}$, H. Ghasemi ${ }^{11}$, R. Handberg ${ }^{1}$,

H. Hansen ${ }^{1}$, A. Hasanzadeh ${ }^{12}$, G. Houdek ${ }^{1}$, C. Johnston ${ }^{3}$, A. B. Justesen ${ }^{1}$,

F. Kahraman Alicavus ${ }^{13,14}$, K. Kotysz ${ }^{7}$, D. Latham ${ }^{15}$, J.M. Matthews ${ }^{16}$, J. Mønster ${ }^{1}$,

E. Niemczura ${ }^{7}$, E. Paunzen ${ }^{17}$, J. P. Sánchez Arias ${ }^{18}$, A. Pigulski ${ }^{7}$, J. Pepper ${ }^{19}$,

T. Richey-Yowell ${ }^{19,20}$, H. Safari ${ }^{12}$, S. Seager ${ }^{21,22,23}$ B. Smalley ${ }^{24}$, T. Shutt ${ }^{25}$, A. Sódor ${ }^{26,27}$, J.-C. Suárez ${ }^{28,29}$, A. Tkachenko ${ }^{3}$, T. $\mathrm{Wu}^{30,31,32}, \mathrm{~K}$. Zwintz ${ }^{33}$, S. Barceló Forteza, ${ }^{34}$, E. Brunsden ${ }^{25}$, Z. Bognár ${ }^{26,27}$, D. L. Buzasi ${ }^{35}$, S. Chowdhury ${ }^{13}$, P. De Cat ${ }^{36}$,

J. A. $\operatorname{Evans}^{24}$, Z. Guo ${ }^{37,13}$, J. A. Guzik ${ }^{38}$, N. Jevtic ${ }^{39}$, P. Lampens ${ }^{36}$, M. Lares Martiz ${ }^{10}$,

C. Lovekin ${ }^{40}$, G. $\mathrm{Li}^{4}$, G. M. Mirouh ${ }^{41}$, D. Mkrtichian ${ }^{42}$, M. J. P. F. G. Monteiro ${ }^{2,43}$ J. M. Nemec ${ }^{44}$, R. Ouazzani ${ }^{6}$, J. Pascual-Granado ${ }^{10}$, D. R. Reese ${ }^{6}$, M. Rieutord ${ }^{45}$, J. R. Rodon ${ }^{10}$, M. Skarka ${ }^{46,47}$, P. Sowicka ${ }^{13}$, I. Stateva ${ }^{48}$, R. Szabó ${ }^{26,27}$, and W.W. Weiss ${ }^{49}$

The authors' affiliations are shown in Appendix B.

Accepted XXX. Received YYY; in original form ZZZ

\begin{abstract}
We present the first asteroseismic results for $\delta$ Scuti and $\gamma$ Doradus stars observed in Sectors 1 and 2 of the TESS mission. We utilise the 2-min cadence TESS data for a sample of 117 stars to classify their behaviour regarding variability and place them in the Hertzsprung-Russell diagram using Gaia DR2 data. Included within our sample are the eponymous members of two pulsator classes, $\gamma$ Doradus and SX Phoenicis. Our sample of pulsating intermediate-mass stars observed by TESS also allows us to confront theoretical models of pulsation driving in the classical instability strip for the first time and show that mixing processes in the outer envelope play an important role. We derive an empirical estimate of $74 \%$ for the relative amplitude suppression factor as a result of the redder TESS passband compared to the Kepler mission using a pulsating eclipsing binary system. Furthermore, our sample contains many highfrequency pulsators, allowing us to probe the frequency variability of hot young $\delta$ Scuti stars, which were lacking in the Kepler mission data set, and identify promising targets for future asteroseismic modelling. The TESS data also allow us to refine the stellar parameters of SX Phoenicis, which is believed to be a blue straggler.
\end{abstract}

Key words: asteroseismology - techniques: photometric: TESS - binaries: chemically peculiar - stars: interiors - stars: variables: $\delta$ Scuti: variables: $\gamma$ Doradus 
stars across the Hertzsprung-Russell (HR) diagram, and ultimately improve our understanding of stellar evolution. In particular, the interior rotation, mixing and angular momentum profiles represent significant uncertainties in theoretical models of stellar structure. All these uncertainties propagate from the main-sequence into later stages as these strongly influence the evolution of a star (Maeder 2009; Meynet et al. 2013; Aerts et al. 2018a). The only observational method for determining these unknowns in models is asteroseismology, which uses stellar oscillations to probe the sub-surface physics of stars (Aerts et al. 2010). Asteroseismology allows us to test internal stellar physics across the entire HR diagram, since different classes of pulsating stars exist at various stages of stellar evolution.

Stellar pulsations in intermediate-mass stars are principally of two main types, characterised by their respective restoring mechanisms: pressure $(\mathrm{p})$ modes, where pressure forces restore perturbations, and gravity (g) modes, where buoyancy does (Aerts et al. 2010). However, for moderately and rapidly rotating stars, the Coriolis force is also important as a restoring force, leading to gravito-inertial modes, or Rossby (r) modes (Saio et al. 2018a). Typically p modes are most sensitive to the stellar envelope whereas g modes probe the near-core region, with pulsations described by spherical harmonics such that each $\mathrm{p}$ or g mode has a radial order, $n$, angular degree, $\ell$, and azimuthal order, $m$.

In the last two decades, space telescopes such as WIRE (Buzasi et al. 2005), MOST (Matthews 2007), CoRoT (Auvergne et al. 2009), Kepler (Koch et al. 2010) and BRITE (Weiss et al. 2014) have provided the necessary continuous, long-term and high-precision time series photometry for the identification of large numbers of individual pulsation modes in single stars. Hence asteroseismology has been successfully applied to hundreds of solar-type and tens of thousands of red giant stars (Chaplin \& Miglio 2013), as well as dozens of pulsating white dwarfs and intermediatemass main-sequence stars (e.g., Aerts et al. 2017, 2018a; Li et al. 2019a).

Among the intermediate-mass main-sequence $\mathrm{A}$ and $\mathrm{F}$ stars, there are different pulsator classes, such as the $\delta$ Sct, $\gamma$ Dor and rapidly oscillating Ap (roAp) stars. These classes are located at the intersection of the main-sequence and the classical instability strip in the HR diagram, and exhibit p modes and/or g modes pulsations (Breger 2000; Aerts et al. 2010). The high-frequency $\left(v \gtrsim 4 \mathrm{~d}^{-1}\right)$, low-radialorder $\mathrm{p}$ modes in $\delta$ Sct stars $^{1}$ are excited by the opacity mechanism operating in the $\mathrm{He}$ II ionisation zone, as well as by turbulent pressure, which has also been shown to be responsible for excitation of moderate radial order p modes within the classical instability strip (Antoci et al. 2014; Xiong et al. 2016). On the other hand, low-frequency $\left(v \lesssim 4 \mathrm{~d}^{-1}\right)$ g modes in $\gamma$ Dor stars are excited by a convective flux modulation mechanism (Guzik et al. 2000; Grigahcène et al. 2010; Dupret et al. 2005). The instability regions of the $\delta$ Sct and $\gamma$ Dor stars partially overlap in the HR diagram (Dupret et al. 2005), and include stars on the pre-

1 The exact boundary between $\mathrm{p}$ and g modes depends on the stellar parameters, and especially the rotation of the star. main-sequence, the main-sequence and post-main-sequence with typically masses in the range of $1.2 \leqslant M \leqslant 2.5 \mathrm{M}_{\odot}{ }^{2}$.

Space telescopes have provided continuous, long-term light curves of tens of thousands of pulsating stars. In particular, Kepler heralded a large and important step forward for asteroseismology of $\delta$ Sct and $\gamma$ Dor stars, since its 4yr light curves provided unprecedented frequency resolution and duty cycle, both of which are necessary for accurate mode identification. An unexpected discovery was that many pulsating $\mathrm{A}$ and $\mathrm{F}$ stars are hybrid pulsators exhibiting both p and g modes (Grigahcène et al. 2010; Uytterhoeven et al. 2011; Balona \& Dziembowski 2011), although pure pulsators of both types do exist (see e.g. Van Reeth et al. 2015a; Bowman 2017; Barceló Forteza et al. 2018; Li et al. 2019a). The high incidence of hybrid stars remains unexplained, particularly if a single excitation mechanism should responsible for both the $\mathrm{p}$ and $\mathrm{g}$ modes in hybrid stars (Balona et al. 2015; Xiong et al. 2016). This incomplete understanding of pulsational excitation in A and F stars is further complicated by the observation that a significant fraction of hybrids, $\gamma$ Dor and $\delta$ Sct stars are hotter than their theoretically predicted instability regions, and that constant stars also exist within the $\delta$ Sct instability domain (Bowman \& Kurtz 2018; Murphy et al. 2019).

A major advance in asteroseismology has been the characterisation of internal rotation profiles of dozens of $\mathrm{A}$ and $\mathrm{F}$ stars using their g mode pulsations (Van Reeth et al. 2016; Aerts et al. 2017; Li et al. 2019a). From these studies, it has been inferred that a significant fraction of intermediatemass main-sequence stars have, at first sight, near-uniform radial rotation profiles (e.g., Kurtz et al. 2014; Saio et al. 2015; Van Reeth et al. 2016, 2018; Ouazzani et al. 2017; Christophe et al. 2018; Li et al. 2019a,b), however in order to draw a final conclusion detailed studies in the manner of, e.g., Ouazzani et al. (2012) and Hatta et al. (2019) are required. Furthermore, the large number of excited g modes in these stars allow the physics of convective core overshooting and chemical mixing to be probed, as well as accurate determinations of stellar masses and ages using forward seismic modelling (Schmid \& Aerts 2016; Aerts et al. 2018b; Mombarg et al. 2019). These studies clearly demonstrate the asteroseismic potential of high quality space-based observations of intermediate-mass stars.

Although the pulsation mode density is typically high in $\delta$ Sct stars hampering mode identification, some stars have been shown to show regularities in their spectra consistent with the so-called large frequency separation which may aid in mode identification and modelling of p modes (see, e.g., García Hernández et al. 2015, 2017; Paparó et al. 2016a,b; Barceló Forteza et al. 2017). The large frequency separation is the separation between consecutive radial order modes of the same degree and is a well-known quantity in the context of Sun and Sun-like stars (Aerts et al. 2010). When using the same quantity for $\delta$ Sct stars, special care must be taken as these stars typically pulsate in lower radial order modes, where the large frequency separation deviates from the one measured at higher radial orders. Nevertheless, it was shown that these patterns are also proportional to the mean density

2 In the case of low metallicity the mass can be lower than $1.2 \mathrm{M}_{\odot}$, see, e,g, Sec. 6.3 
of the stars (Suárez et al. 2014) which was observationally confirmed (García Hernández et al. 2015, 2017).

More recently, the successful launch of TESS and the delivery of the first TESS mission data (Ricker et al. 2014, 2015), which are in the southern ecliptic hemisphere, provide new insight into pulsation and rotation in intermediate-mass stars (e.g., Cunha et al. 2019; Sikora et al. 2019). The primary mission of TESS follows the groundwork of the Kepler mission, and aims to detect planetary transits in the brightest stars across the sky. TESS data cover almost the entire sky $(|b|>6 \mathrm{deg})$ at a cadence of $30 \mathrm{~min}$ and are publicly available to the community in the form of full frame images. However, a sub-sample of stars were selected to be observed at a 2-min cadence in each TESS observing sector and light curves of these stars are being delivered to the community a few months after observations have been obtained. Therefore, TESS provides a unique homogenous data set to test and constrain much of the currently unknown physics inside main-sequence $\mathrm{A}$ and $\mathrm{F}$ stars.

In this paper, we demonstrate the asteroseismic potential of TESS for the study of intermediate-mass stars, specifically for $\delta$ Sct and $\gamma$ Dor stars. We show a selection of different types of pulsator classes, including some of the class prototypes, and the significant advantages and inference that can be achieved using TESS mission data. In addition, we show how theoretical models regarding the excitation of pulsations in $\mathrm{A}$ and $\mathrm{F}$ stars can be tested. In the following subsections, we briefly outline the different pulsator classes within the $\mathrm{A}$ and $\mathrm{F}$ stars.

\section{THE PULSATION ZOO OF A AND F STARS}

It is estimated that approximately half of the stars within the classical instability strip are pulsating at the $\mu$ mag photometric precision provided by space telescopes (Balona \& Dziembowski 2011; Murphy et al. 2019). It remains a mystery why the other half do not show pulsations, especially at that precision. Among the intermediate-mass stars there is a veritable zoo of different pulsator classes. The heterogeneity of variability in these stars allows us to test different physical phenomena, such as rotation, diffusion, and convection. Obviously, this is because the differences in observed pulsation characteristics are caused by differences in stellar structure, evolutionary stage and/or metallicity. Here, we briefly discuss the zoo of pulsating A and F stars.

\subsection{Ap and Am stars}

Stars within the $\delta$ Sct instability strip are predicted to pulsate in $\mathrm{p}$ modes excited by the opacity mechanism operating in the He II ionisation zone (Cox 1963; Aerts et al. 2010) as well as by turbulent pressure (e.g., Antoci et al. 2014). However, whether a star is unstable to pulsation strongly depends on mixing processes, such as rotation or the presence of a strong magnetic field, which can suppress the excitation of heat-driven (opacity mechanism) pulsation modes.

Slow rotation in intermediate-mass stars is typically caused by one of two mechanisms: (i) magnetic braking by a large-scale magnetic field; or (ii) tidal braking due to tidal forces caused by a close companion. Approximately 10 per cent of A-type stars are observed to have a strong, large-scale magnetic field, which is likely of fossil origin and causes magnetic braking during the pre-main-sequence stage (Stȩpień 2000). The slow rotation and strong magnetic field allow atomic diffusion - radiative levitation and gravitational settling of different ions - to give rise to surface spectral peculiarities (Abt 2000), which define the sub-classes of the magnetic Ap stars, and suppress the excitation of low radial-order p modes by the opacity mechanism (Saio 2005). However, a small fraction of these magnetic Ap stars pulsate in high-radial order modes; these are the rapidly oscillating Ap (roAp) stars (Kurtz 1982). The excitation of low-degree, high-radial order magneto-acoustic modes in roAp stars provides the opportunity to study the effects of pulsation and rotation in the presence of strong magnetic fields (Cunha \& Gough 2000; Cunha 2001; Bigot \& Dziembowski 2002; Cunha 2006; Saio \& Gautschy 2004; Saio 2005). We refer the reader to Cunha et al. (2019) for the first results on roAp stars based on TESS data.

The second main sub-class of slowly rotating $\mathrm{A}$ and $\mathrm{F}$ stars are the non-magnetic Am stars (Conti 1970; Breger 1970; Kurtz 1978; Aurière et al. 2010). The presence of a close companion causes tidal braking such that atomic diffusion and gravitational settling separate chemical elements in the stellar envelope, deplete helium from the excitation region and inhibit the efficiency of pulsational excitation (Michaud 1970; Baglin et al. 1973; Turcotte et al. 2000). However, recent studies focussed on the incidence of pulsations in Am stars have found a non-negligible fraction pulsating in p modes (Smalley et al. 2011, 2017) with a significant amount of excitation connected to the turbulent pressure in the hydrogen rather than the He II ionisation layer (Antoci et al. 2014; Smalley et al. 2017). We present our analysis of TESS photometry of pulsating Am stars in Section 5.

\subsection{Metal-poor stars}

Among the A-type pulsators are two classes of metal-poor stars: the $\lambda$ Boo stars and SX Phe stars. The $\lambda$ Boo stars are a spectroscopically defined class in which refractory chemical elements - those with high condensation temperatures in pre-stellar disks - are underabundant by 0.5 to $2 \mathrm{dex}$, but volatile elements such as $\mathrm{C}$ and $\mathrm{O}$ show solar abundance (Heiter 2002; Andrievsky et al. 2002). The peculiarity of these stars is believed to originate from selective accretion of gas with little dust, but the specifics remain uncertain (Venn \& Lambert 1990; King 1994; Kama et al. 2015), and it is not clear whether the peculiarity is restricted to surface layers only. Approximately 2 per cent of A stars are $\lambda$ Boo stars (Gray \& Corbally 1998), for which a catalogue has been compiled by Murphy et al. (2015). At present, there is no known difference between the pulsational characteristics of $\lambda$ Boo $\delta$ Sct stars and 'normal' $\delta$ Sct stars.

SX Phe stars, on the other hand, are not a spectroscopically defined class but comprise Population II $\delta$ Sct stars, though not all of them are metal poor (Nemec et al. 2017). Many SX Phe stars are identified as blue stragglers in clusters, while those in the field are often identified kinematically. The dominant pulsations in SX Phe stars are typically high-amplitude radial modes (Nemec \& Mateo 1990; Templeton et al. 2002; Murphy et al. 2013) but can also have low amplitudes (Kurtz et al. 2014). We analyse TESS data for SX Phe itself in section 6.3. 


\subsection{High-amplitude $\delta$ Sct stars}

High-amplitude $\delta$ Scuti stars (HADS) are usually recognized by their large amplitude, non-sinusoidal light curves and small rotational velocities, but their most essential property is the dominant presence of the fundamental and/or first overtone radial mode(s) (McNamara 2000; Breger 2000). In the HR diagram, ground-based observations indicated that HADS occupy a narrow strip in the centre of $\delta$ Sct instability region, perhaps because the efficiency of mode excitation is maximised in this region (Petersen \& ChristensenDalsgaard 1996). However, space photometry revealed that some HADS can be found throughout the entire instability strip (Balona 2016).

Some studies have investigated whether the properties of HADS could be explained by them being in a post-main-sequence stage of stellar evolution (e.g. Petersen \& Christensen-Dalsgaard 1996; Bowman 2017), since they obey a period-luminosity relation which allows an independent distance determination for some stellar systems (e.g., Nemec et al. 1994; McNamara et al. 2007; Cohen \& Sarajedini 2012; Kopacki 2015; Ziaali et al. 2019), such as globular clusters in which SX Phe stars are abundant. However, there remains no clear consensus on a physical difference between HADS and their lower amplitude $\delta$ Sct counterparts. Important remaining questions concerning HADS include: the excitation (e.g., Poretti et al. 2011); the relation between the single and double-mode HADS and the stellar parameters; and whether the pulsational properties of SX Phe stars differ from those of Population I HADS. The all-sky TESS sample of HADS will be particularly useful for addressing these questions and will extend the study of a handful of Kepler stars, particularly the seismic modelling of HADS such as those by Breger et al. (2011) and Balona et al. (2012).

The TESS sample of HADS and SX Phe stars in Sectors 1 and 2 includes at least 19 stars proposed for 2-min cadence observations based on a review of the ASAS-3 (Pojmański 1997, 2002) database (Pigulski \& Kotysz, in preparation). The sample includes several known HADS, e.g., ZZ Mic, RS Gru, BS Aqr and SX Phe itself (see Sect. 6.3). An example of a known HADS star observed by TESS, HD 224852 (TIC 355687188 ), is presented in detail in Sect. 6.6.

\subsection{Pre-main-sequence stars}

Intermediate-mass pre-main-sequence stars within the instability regions can become unstable to $\mathrm{p}$ and g-modes during their evolution from the birthline to the zero-age mainsequence (ZAMS). Pre-main-sequence stars differ from their main-sequence and post main-sequence analogues of similar mass because of their interior structure (Marconi \& Palla 1998). Since pulsation modes carry information about the inner parts of stars and show a different pattern for stars on the pre- or the post-main-sequence phases (Suran et al. 2001 ), it is possible to use asteroseismology to constrain the evolutionary stage of a star (Guenther et al. 2007). This is important because the evolutionary tracks for stars before and after the ZAMS intersect in this part of the HR diagram making it impossible to constrain a star's evolutionary stage from only its effective temperature and luminosity.

In general, without asteroseismology, the pre-mainsequence stage of a star in this region of the HR diagram can only be assessed using certain observational features as indicators. Such indicators for young stars are emission lines in the spectra, infrared or ultraviolet excess, X-ray fluxes, and membership of a young open cluster or a star-forming region, i.e., younger than approximately 10 million years (Sloan et al. 2004). However, some of these features can also be misleading, since the comparatively old, low-mass asymptotic giant branch (AGB) and post-AGB stars show similar observational properties and populate the same region in the HR diagram as young stellar objects (Kamath et al. 2014). Therefore, special care has to be taken when investigating the potential early evolutionary stage of a given star.

Currently, about 80 pre-main-sequence stars are known to be p- and g-mode pulsators of three different types: Slowly Pulsating B (SPB) stars (Gruber et al. 2012), $\delta$ Sct stars (e.g., Zwintz et al. 2014) and $\gamma$ Dor stars (Zwintz et al. 2013). Based on their basic properties, such as spectral types and effective temperature, TIC 150394126 and TIC 382551468 are two candidates for $\delta$ Sct and $\gamma$ Dor pulsations. The former has an infrared excesse (Sloan et al. 2004) and the latter is potentially an Herbig Ae/Be type star, identifying them as likely being in their pre-main-sequence evolutionary stages. We analyse these stars in Section 6.5.

\section{FREQUENCY ANALYSES OF TESS DATA}

The TESS data analysed in this work are the 2-min 'Simple Aperture Photometry' (SAP) light curves provided by the TESS Science Team, which are publicly available from the Mikulski Archive for Space Telescopes (MAST) ${ }^{3}$. As part of the framework of the asteroseismic working group on pulsating stars of spectral type A and F within the TESS Asteroseismic Science Consortium (TASC) ${ }^{4}, 117$ known pulsating stars were allocated observing slots at a 2-min cadence by the TESS Mission in Sectors 1 and 2 of its 1-yr observing campaign in the southern ecliptic hemisphere. There were more $\delta$ Sct stars observed in the first two sectors, but for this study we decided to concentrate on those that were allocated to the TASC working group on A and F stars (TASC WG $4)$. Because g modes have long periods, we did not specifically include known $\gamma$ Dor stars in our 2-min target list, since these stars will be observed with a cadence of $30 \mathrm{~min}$, which is sufficient to determine their frequencies. We discuss one star that was not observed during Sectors 1 and 2 later on. This target is the eponymous star $\gamma$ Doradus and is described in Section 6.7.

We analysed the TESS light curves of our sample in different independent groups, using various frequency extraction software routines. Commonly used programs to perform frequency analysis of light curves are PERIOD04 (Lenz \& Breger 2004) and SigSpec (Reegen 2007), while the KU Leuven and Aarhus University ECHO (Extraction of CoHerent Oscillations) pipelines were used also. We discuss these pipelines in Sections 3.1 and 3.2, and conclude that they yield compatible results for peaks in the amplitude spectrum with signal-to-noise $(\mathrm{S} / \mathrm{N}) \geqslant 4$. A comparison of the significant frequencies identified in two stars by the Leuven and Aarhus pipelines is shown in Fig. 1. We plan to compile

${ }^{3}$ https://archive.stsci.edu ${ }^{4}$ https://tasoc.dk 

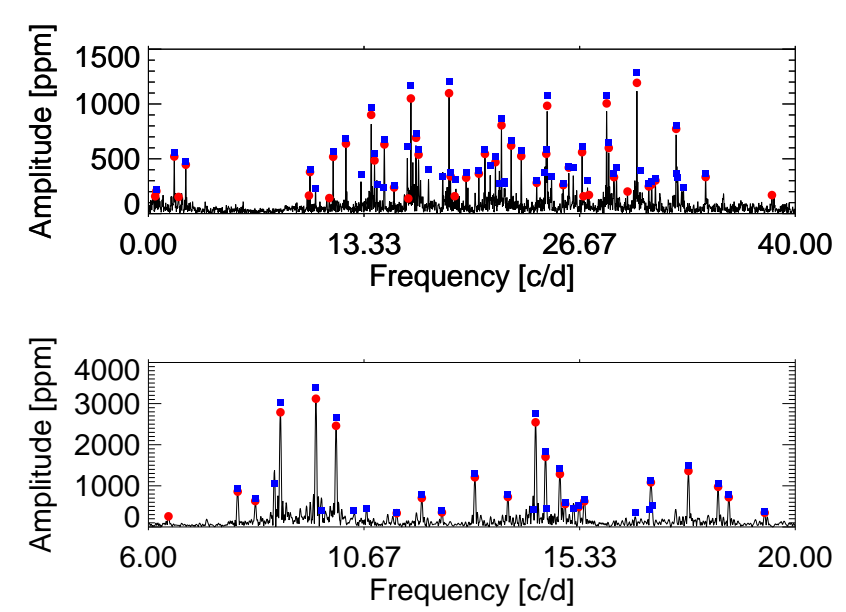

Figure 1. Comparison between the frequency extraction pipelines from Leuven (blue squares) and Aarhus (red circles) showing good agreement between the two different pipelines. The amplitude values are offset for clarity. Upper panel: HD 210111 (TIC 229059574). Lower Panel: TX Ret (TIC 38587180).

a catalogue of pulsating A and F stars observed by TESS at the 2-min cadence in the entire southern ecliptic hemisphere, which will include all significant pulsation frequencies and identification of their combination frequencies.

\subsection{The KU Leuven pipeline}

The KU Leuven iterative pre-whitening pipeline was used to extract significant periodic variability in time series data. It uses Lomb-Scargle (Scargle 1982) periodogram and multifrequency non-linear least-squares optimisation to extract significant frequencies and their associated amplitudes and phases. Uncertainties on frequencies, amplitudes and phases are calculated according to Montgomery \& O'Donoghue (1999), and corrected for the correlated nature of oversampled time series data following Schwarzenberg-Czerny (2003). The pipeline was developed by Degroote et al. (2009), and improved by Pápics et al. (2012) and Van Reeth et al. (2015b); detailed discussions and applications to pulsating stars observed by CoRoT and Kepler were provided by these studies.

With this pipeline, peaks in the amplitude spectrum can be extracted in order of decreasing amplitude or decreasing $\mathrm{S} / \mathrm{N}$, as specified by the user. Normally, a lower limit of $\mathrm{S} / \mathrm{N}$ $\geqslant 4$ (Breger et al. 1993) is chosen, where the noise is calculated as the average amplitude in a symmetric $1-\mathrm{d}^{-1}$ window in the residual periodogram after pre-whitening. From extensive testing, it has been found that using a window around the extracted peak for calculating a local noise estimate (and hence the $\mathrm{S} / \mathrm{N}$ ) is more pragmatic and reliable for iterative pre-whitening than using a $p$-value (Pápics et al. 2012). Otherwise, hundreds or even thousands of individual frequencies can be found to be statistically significant, yet with an increasing probability of an artificial frequency having been injected during the prewhitening process into a light curve when the number of iterations becomes large. Typically, this limit is a few hundred frequency extraction iterations for a 4-yr Kepler light curve of a $\delta$ Sct or $\gamma$ Dor star, but this depends on the quality and lengths of the data, and assumes that peaks are coherent and so varies from star to star.

\subsection{ECHO}

The ECHO program (Extraction of CoHerent Oscillations) was developed at Aarhus University. It extracts coherent signals from photometric light curves using an optimized, iterative pre-whitening technique. Coherent signals are iteratively identified using a Lomb-Scargle periodogram that is calculated using the Press \& Rybicki (1989) algorithm, which allows a fast computation for unevenly sampled data. The frequency, amplitude and phase of an oscillation is computed using the algorithm described by Frandsen et al. (1995). The statistical uncertainties on the amplitudes, frequencies and phases are calculated according to Montgomery \& O'Donoghue (1999). The statistical significances of the extracted signals are estimated from the periodogram using Scargle's significance criteron (Scargle 1982). The extracted signals are continuously optimized at each iteration, taking into account the influence of one signal on another. This is done by reinserting previously extracted signals back into the pre-whitened light curve and redetermining the maximum amplitude of the peak. This may be slightly different from the previously found value, due to the influence of neighbouring peaks (Handberg 2013).

Using simulated light curves, Balona (2014) showed that iterative pre-whitening can lead to a very large number of false detections. This is because imperfect subtraction of signals will inject new, statistically significant signals into the light curve. For this reason, the reliability of each extracted oscillation is determined by comparing the amplitude of an extracted signal $A_{\mathrm{f}}^{\mathrm{ex}}$ to the corresponding amplitude $A_{\mathrm{f}}^{\mathrm{og}}$ in the unaltered, original light curve. If the amplitudes do not agree within a certain limit $\alpha$, such that

$$
\alpha<\frac{A_{\mathrm{f}}^{\mathrm{ex}}}{A_{\mathrm{f}}^{\mathrm{og}}}<\frac{1}{\alpha},
$$

the extracted signal is rejected. From simulated light curves, we found that $\alpha=0.75$ is a suitable value, keeping the number of false detections to a minimum while still extracting most of the real signals present in the light curve. These simulations were based on 4 years of Kepler data. For shorter observations, our tests showed that a more conservative factor of $\alpha=0.8$ is required. This method is similar to that used by Van Reeth et al. (2015b), although they started with $\alpha=0.5$ and allowed it to vary during pre-whitening. In our use of the Leuven and ECHO pipelines, we enforced a maximum of number of 100 frequencies that could be extracted, although it was not typically needed since peaks became insignificant before this criterion was met. This is because the pre-whitening of so many peaks can introduce additional signals, and make the results unreliable.

In addition, we must be aware that observations of 'only' 27-56 days may not sufficient to resolve closely-spaced frequencies clearly known to be present in many $\delta$ Sct and $\gamma$ Dor stars. Kallinger et al. (2017) have gone beyond the formal frequency resolution of $1 / \Delta \mathrm{T}$ in their frequency anal- 
$\mathrm{ysis}^{5}$, using a probabilistic approach. We, however, prefer to use the traditional Rayleigh criterion because extracting unresolved frequencies, can lead to over-interpreting the data and wrong input for asteroseismic modelling. We recommend using formally unresolved peaks with extreme caution, and only based on careful testing and the use of additional independent information (e.g., for roAp stars, where an assumption of the oblique pulsator model requires exact splitting in the rotational multiplets). The statistical significance of extracted peaks is an important topic that goes beyond the scope of this paper as we are only showing a first view into TESS $\delta$ Sct and $\gamma$ Dor stars. At this stage we are expanding ECHO to also include the 'traditional' SNR test.

\section{TARGET STARS}

In this section, we present the details of all TASC WG4 $\mathrm{A}$ and $\mathrm{F}$ stars that are not bona fide or suspected Ap or roAp stars observed in a 2-min cadence in Sectors 1 and 2 . The variability type for each star was identified, based on TESS data, by visual inspection of the light curves and their Fourier spectra. Spectroscopic and photometric parameters from the literature for all the stars, where available, are assembled in Table A1 in the appendices, including claimed detections of binarity. Note that here we do not evaluate the robustness of these literature values and leave it to the reader to investigate these in detail.

In Table 2, we list the newly derived effective temperatures using the spectral energy distribution method (SED) (see Section 4.1), new spectroscopically derived parameters (see Section 4.2), the dereddened luminosities from Gaia and Hipparcos data (Section 4.3), and the TESS stellar variability type. The latter is one of the following categories: (1) $\delta$ Sct star: $v \geqslant 4 \mathrm{~d}^{-1} ;(2) \gamma$ Dor star: $v \leqslant 4 \mathrm{~d}^{-1}$; (3) hybrid star: when frequencies were detected in the $\gamma$ Dor and the $\delta$ Sct frequency ranges. Note that in many cases the peaks in the $\gamma$ Dor range were not resolved and more data are required to fully characterise the frequencies. (4) binarity: eclipses and/or ellipsoidal modulation were detected; (5) rotational variable (rot): variability is consistent with rotational modulation of spotted stellar surfaces. (6) no detection: no significant variability was detected. We also note that $v$ Pic (TIC 260416268) was observed during sectors 1 and 2 but the amplitude differs significantly between the sectors suggesting a problem in the data extraction.

\footnotetext{
${ }^{5}$ where $\Delta \mathrm{T}$ is the lengths of the data set.
} 
Table 1: Stellar parameters for the TESS $\delta$ Sct and $\gamma$ Dor pulsators observed at 2-min cadence during the first two pointings (Sectors 1 and 2) in the ecliptic Southern hemisphere. The columns indicate: the TESS Input Catalogue (TIC) number, the TESS magnitude, an alternative identifier of the star, spectroscopic (Spec.) and photometric (Phot) effective temperatures and $\log g$ values and their associated uncertainties from literature respectively. The reference is indicated next to each value. In addition, we list the projected rotational velocity and binarity status as available in literature. The full table is available in the online version in the appendix.

\begin{tabular}{|c|c|c|c|c|c|c|c|c|c|c|c|c|c|}
\hline TIC & $\begin{array}{c}\text { TESS } \\
\text { mag }\end{array}$ & $\begin{array}{l}\text { alternative } \\
\text { identifier }\end{array}$ & $\begin{array}{l}T_{\text {eff }}[K] \\
\text { Spec. }\end{array}$ & $\begin{array}{l}\sigma_{T_{\text {eff }}} \\
\text { Spec. }\end{array}$ & $\begin{array}{l}T_{\text {eff }}[K] \\
\text { Phot. }\end{array}$ & $\begin{array}{l}\sigma_{T_{\text {eff }}} \\
\text { Phot. }\end{array}$ & $\begin{array}{l}\log g \\
\text { Spec. }\end{array}$ & $\begin{array}{l}\sigma_{\log g} \\
\text { Spec. }\end{array}$ & $\begin{array}{l}\log g \\
\text { Phot. }\end{array}$ & $\begin{array}{l}\sigma_{\log g} \\
\text { Phot. }\end{array}$ & $\begin{array}{c}v \sin i \\
{\left[\mathrm{~km} \mathrm{~s}^{-1}\right]}\end{array}$ & $\begin{array}{c}\sigma_{\mathrm{vsini}} \\
{\left[\mathrm{km} \mathrm{s}^{-1}\right]}\end{array}$ & binarity \\
\hline 9632550 & 9.06 & BS Aqr & $7245^{1}$ & $75^{1}$ & - & - & $3.82^{1}$ & $0.12^{1}$ & - & - & $23^{1}$ & - & - \\
\hline 12470841 & 8.44 & HD 923 & - & - & $7832^{2}$ & - & - & - & - & - & - & - & - \\
\hline 12784216 & 8.92 & HD 213125 & - & - & $6519^{3}$ & $178^{3}$ & - & - & $4.03^{3}$ & $0.26^{3}$ & - & - & - \\
\hline 12974182 & 6.78 & HD 218003 & $7471^{4}$ & $95^{4}$ & $7584^{5}$ & $208^{5}$ & $3.86^{4}$ & $0.18^{4}$ & $4.05^{5}$ & - & - & - & - \\
\hline$\cdots$ & $\cdots$ & $\cdots$ & $\cdots$ & $\cdots$ & $\cdots$ & $\cdots$ & $\cdots$ & $\cdots$ & $\cdots$ & $\cdots$ & $\cdots$ & $\cdots$ & $\cdots$ \\
\hline
\end{tabular}

Table 2: Stellar parameters and variability type of the 2-min cadence TESS $\delta$ Scuti and $\gamma$ Dor pulsators determined in this work. In this table we list the TESS Input Catalogue (TIC) name, an alternative name for each star, the effective temperatures derived using the SED method (Section 4.1), the luminosities derived based on the Gaia and when available Hipparcos parallaxes and the respective uncertainties (Section 4.3). In column 9 we describe the variability type determined from the TESS data. The question mark means that the variability type is uncertain (e.g. due to unresolved peaks). HAGD denotes a High Amplitude $\gamma$ Dor star. The last column describes the chemical peculiarity as described in Renson \& Manfroid (2009). The full table is available in the online version in the appendix.

\begin{tabular}{|c|c|c|c|c|c|c|c|c|c|}
\hline TIC & $\begin{array}{c}\text { alternative } \\
\text { identifier }\end{array}$ & $\begin{array}{c}T_{\text {eff }}[\mathrm{K}] \\
\text { SED }\end{array}$ & $\overline{\sigma T_{\text {eff }}}$ & $\begin{array}{c}\log \left(\mathrm{L} / \mathrm{L}_{\odot}\right) \\
\text { Gaia }\end{array}$ & $\sigma \log \left(\mathrm{L} / \mathrm{L}_{\odot}\right)$ & $\begin{array}{c}\log \left(\mathrm{L} / \mathrm{L}_{\odot}\right) \\
\text { HIPP. }\end{array}$ & $\sigma \log \left(\mathrm{L} / \mathrm{L}_{\odot}\right)$ & $\begin{array}{l}\text { variability } \\
\text { type }\end{array}$ & $\begin{array}{c}\text { chem. } \\
\text { pec. }\end{array}$ \\
\hline 9632550 & BS Aqr & 6760 & 140 & 1.62 & 0.03 & 1.96 & 0.94 & HADS & \\
\hline 12470841 & HD 923 & 8090 & 230 & 1.64 & 0.02 & 2.01 & 0.43 & one large peak only & \\
\hline 12784216 & HD 213125 & 6390 & 120 & 1.14 & 0.01 & & & $\delta$ Sct/hybrid? & \\
\hline 12974182 & HD 218003 & 7670 & 170 & 1.103 & 0.008 & 1.08 & 0.04 & rot?/binary? & \\
\hline
\end{tabular}

3 Huber et al. (2016)

5 McDonald et al. (2017) 


\subsection{Effective temperatures derived from Spectral Energy Distributions}

Effective temperature can be determined from the stellar spectral energy distribution (SED). For our target stars these were constructed from literature photometry, using, $B$ and $V$ magnitudes from either Tycho (Hoeg et al. 1997) or APASS9 (Henden et al. 2015), USNO-B1 $R$ magnitudes (Monet et al. 2003), $J, H$ and $K$ from 2MASS (Skrutskie et al. 2006), supplemented with CMC14 $r^{\prime}$ (Evans et al. 2002) and APASS9 $g^{\prime}, r^{\prime}, i^{\prime}$ photometry.

Stellar energy distributions can be significantly affected by interstellar reddening, which was calculated as described in Section 4.3 The SEDs were de-reddened using the analytical extinction fit of (Howarth 1983). The stellar $T_{\text {eff }}$ values were determined by fitting solar-composition Kurucz (1993) model fluxes to the de-reddening SEDs. The model fluxes were convolved with photometric filter response functions. A weighted Levenberg-Marquardt non-linear least-squares fitting procedure was used to find the solution that minimized the difference between the observed and model fluxes. Since $\log g$ is poorly constrained by our SEDs, we fixed $\log g=4.0$ for all the fits. The uncertainties in $T_{\text {eff }}$ includes the formal least-squares error and adopted uncertainties in $E(B-V)$ of \pm 0.02 and $[M / H]$ of \pm 0.5 added in quadrature.

\subsection{New spectroscopic determinations of $T_{\text {eff }}$ based on archival data}

Spectroscopic data were taken from the ESO archive ${ }^{6}$. We used medium and high signal-to-noise ratio $(\geqslant 65)$ spectra from FEROS (Fiber-fed Extended Range Optical Spectrograph; R 48000), HARPS (High Accuracy Radial velocity Planet Searcher, R 115000), and UVES (Ultraviolet and Visual Echelle Spectrograph, R 65000). Observational details are given in Table 3 .

We applied two methods to derive spectroscopic parameters for the 14 stars that had available archival data. For the first method we used, the hydrostatic, plane-parallel lineblanketed, local thermodynamic equilibrium ATLAS9 model atmospheres (Kurucz 1993). The synthetic spectra were generated by using the SYNTHE code (Kurucz \& Avrett 1981). The projected rotational velocities, $v \sin i$, were calculated by fitting the profiles of non-blended metal lines (Gray 2008). The $\mathrm{H} \beta$ lines were used to derive $T_{\text {eff }}$ by considering the minimum difference between the synthetic and observed spectra. In this analysis, a solar metallicity and the obtained $v \sin i$ values were fixed. In addition, we assumed $\log g=4.0$ (dex). This was done because the hydrogen lines are not sensitive to $\log g$ for stars with $T_{\text {eff }} \leqslant 8000 \mathrm{~K}$ (Heiter et al. 2002). Our results are listed in Table 4 , with $1 \sigma$ uncertainties.

In addition, the Grid Search in Stellar Parameters (GSSP; Tkachenko (2015)) software package was used as an alternative method for determining the atmospheric parameters of our stars. GSSP is a multi-purpose spectrum analysis tool that is designed for precision spectrum analysis of single and binary stars. As the name suggests, the method is based on a grid-search in 6-D parameter space (metallicity $[\mathrm{M} / \mathrm{H}], T_{\text {eff }}, \log g$, micro- (vmicro) and macro-turbulent $\left(\mathrm{v}_{\text {macro }}\right)$ velocities, and $v \sin i$ ) utilising atmospheric models

6 http://archive.eso.org/cms.html
Table 3. Spectroscopic data.

\begin{tabular}{rlllrc}
\hline \multicolumn{1}{c}{ TIC } & $\begin{array}{c}\text { alternative } \\
\text { name }\end{array}$ & Instrument & Observation & S/N & $\begin{array}{c}\text { Nr. of } \\
\text { spectra }\end{array}$ \\
\hline 9632550 & BS Aqr & FEROS & 2007 July & 200 & 4 \\
32197339 & HD 210139 & HARPS & 2005 July & 105 & 11 \\
38587180 & TX Ret & HARPS & 2011 Dec. & 270 & 1 \\
52258534 & BG Hyi & HARPS & 2011 July & 125 & 2 \\
139825582 & CF Ind & HARPS & 2011 July & 190 & 1 \\
183595451 & AI Scl & UVES & 2001 Dec. & 280 & 1 \\
224285325 & SX Phe & FEROS & 2007 June & 330 & 6 \\
229154157 & HD 11667 & HARPS & 2006 Nov. & 65 & 2 \\
253917376 & UV PsA & HARPS & 2011 July & 150 & 1 \\
265566844 & BX Ind & HARPS & 2011 July & 125 & 1 \\
303584611 & BS Scl & HARPS & 2011 July & 205 & 2 \\
355687188 & HD 224852 & FEROS & 2007 June & 100 & 8 \\
382551468 & CD-53 251 & HARPS & 2008 Nov. & 65 & 28 \\
394015973 & BE Ind & HARPS & 2011 July & 150 & 1 \\
& & & & &
\end{tabular}

and synthetic spectra. Each synthetic spectrum in a grid is compared to the normalised observed spectrum of a star with the chi-square $\left(\chi^{2}\right)$ merit function being used to judge the goodness of fit. Parameter errors are computed from $\chi^{2}$ statistics by projecting all grid points onto each individual parameter so that correlations are taken into account. GSSP employs the SynthV radiative transfer code (Tsymbal 1996) for calculation of synthetic spectra and LLMODELS software package (Shulyak et al. 2004) for computation of atmosphere models. Both codes operate under the assumption of local thermodynamical equilibrium and allow for extra functionality such as vertical stratification of elemental abundances in the atmosphere of a star. The $\log g$ value is not well constrained in the temperature range in question, which is the reason for fixing it to $4.0 \mathrm{dex}$ (as was also done in the previous method). This is because the hydrogen Balmer lines are largely insensitive to changes in the surface gravity in this particular temperature regime, while significant broadening of metal lines and correlations of $\log g$ with $[\mathrm{M} / \mathrm{H}]$ and $\mathrm{v}_{\text {micro }}$ results in typical uncertainties as large as $0.3-0.5$ dex.

The photometric and spectroscopic effective temperatures determined here typically agree within the uncertainties. There are few exceptions all of which are either (spectroscopic) binaries or HADS. For the latter the observed $\mathrm{T}_{\text {eff }}$ depends on the pulsation cycle (see Sec. 6.3 for a discussion).

\subsection{Luminosities derived from the Gaia parallaxes}

We calculated the absolute magnitude and luminosity for each target (see Table 2) using the parallaxes from both the re-reduced Hipparcos data (van Leeuwen 2007) and Gaia DR2 (Lindegren et al. 2016). This was done to check for biases in our parameters, especially for nearby and bright stars. All but two stars from our sample (HD 4125 and $\alpha$ Pic) have Gaia DR2 parallaxes, but only 65 stars have Hipparcos parallaxes including HD 4125 and $\alpha$ Pic.

To estimate the total interstellar extinction, we used several published reddening maps (Arenou et al. 2018; Chen et al. 1998; Schlegel et al. 1998; Drimmel et al. 2003; Green et al. 2015, 2018). The distances and their uncertainties were 
Table 4. Spectroscopic parameters determined using two different methods as described in detail in Section 4.2. In columns three and four we list the $T_{\text {eff }}$ determined from the hydrogen lines and GSSP methods, respectively. The parameters agree within their uncertainties. The $v \sin i$ values were also calculated by two different teams, and agree well within the error bars (here we chose to display the values determined with the GSSP method). The metallicity and vmicro were determined using the GSSP code. In the last column LPV stands for line profile variations. The asymmetries observed in the H $\beta$ line in HD 224852 are likely due to the data extraction pipeline.

\begin{tabular}{|c|c|c|c|c|c|c|c|}
\hline TIC & $\begin{array}{l}\text { alternative } \\
\text { name }\end{array}$ & $\begin{array}{l}T_{\text {eff }}[\mathrm{K}] \\
\mathrm{H} \text { lines }\end{array}$ & $\begin{array}{c}T_{\text {eff }}[\mathrm{K}] \\
\text { GSSP }\end{array}$ & $\begin{array}{c}v \sin i \\
{\left[\mathrm{~km} \mathrm{~s}^{-1}\right]}\end{array}$ & {$[\mathrm{M} / \mathrm{H}]$} & $\begin{array}{c}\mathrm{v}_{\text {micro }} \\
{\left[\mathrm{km} \mathrm{s}^{-1}\right]}\end{array}$ & comments \\
\hline 9632550 & BS Aqr & $7100 \pm 150$ & $7240 \pm 290$ & $21 \pm 6$ & $-0.2 \pm 0.3$ & $3.3 \pm 2.5$ & SB2 \\
\hline 32197339 & HD 210139 & $7600 \pm 200$ & $7960 \pm 95$ & $8 \pm 1$ & $-0.05 \pm 0.08$ & $2.9 \pm 0.6$ & pronounced LPVs \\
\hline 38587180 & TX Ret & $7200 \pm 150$ & $7310 \pm 40$ & $70 \pm 3$ & $-0.01 \pm 0.04$ & $3.8 \pm 0.5$ & \\
\hline 52258534 & BG Hyi & $6900 \pm 120$ & $6980 \pm 50$ & $25 \pm 1$ & $-0.35 \pm 0.07$ & $4.3 \pm 0.5$ & \\
\hline 139825582 & $\mathrm{CF}$ Ind & $7100 \pm 150$ & $7090 \pm 40$ & $227 \pm 13$ & $-0.22 \pm 0.06$ & $3.6 \pm 0.5$ & pronounced LPVs \\
\hline 183595451 & AI Scl & $7300 \pm 120$ & $7315 \pm 40$ & $102 \pm 5$ & $-0.05 \pm 0.07$ & $4.0 \pm 0.6$ & \\
\hline 224285325 & SX Phe & $7500 \pm 150$ & $7500 \pm 115$ & $18 \pm 2$ & $-0.1 \pm 0.2$ & $1.9 \pm 0.4$ & \\
\hline 229154157 & HD 11667 & $7300 \pm 200$ & $7185 \pm 75$ & $16 \pm 1$ & $-0.06 \pm 0.07$ & $3.1 \pm 0.5$ & SB2, with LPVs \\
\hline 253917376 & UV PsA & $7000 \pm 200$ & $7025 \pm 50$ & $76 \pm 4$ & $-0.16 \pm 0.06$ & $4.0 \pm 0.5$ & \\
\hline 265566844 & BX Ind & $6800 \pm 220$ & $6790 \pm 60$ & $5 \pm 1$ & $0.04 \pm 0.06$ & $1.3 \pm 0.3$ & \\
\hline 303584611 & BS Scl & $7900 \pm 150$ & $7955 \pm 55$ & $32 \pm 1$ & $-0.08 \pm 0.05$ & $3.2 \pm 0.4$ & \\
\hline 355687188 & HD 224852 & $7000 \pm 150$ & $7335 \pm 90$ & $14 \pm 1$ & $-0.04 \pm 0.08$ & $3.0 \pm 0.5$ & asymmetric $\mathrm{H} \beta$ \\
\hline 382551468 & CD-53 251 & $6500 \pm 200$ & $6800 \pm 80$ & $13 \pm 1$ & $-0.08 \pm 0.07$ & $2.0 \pm 0.4$ & pronounced LPVs \\
\hline 394015973 & BE Ind & $7500 \pm 200$ & $7500 \pm 50$ & $45 \pm 2$ & $0.06 \pm 0.06$ & $3.3 \pm 0.5$ & \\
\hline
\end{tabular}

calculated directly from the parallaxes. Almost all stars are within $1 \mathrm{kpc}$ from the Sun, which means that the extinction is small but not negligible (total absorption $A_{\mathrm{V}}<0.25 \mathrm{mag}$, with a mean and median of 0.05 and $0.04 \mathrm{mag}$, respectively). We calculated a mean value from the above-listed references, which are all consistent within $0.01 \mathrm{mag}$. This value of 0.01 mag was adopted as the extinction uncertainty for all targets. We chose to do so because there are no estimates of the individual errors from the different maps and methods.

To calculate luminosities for our targets, we used the bolometric corrections for the $V$ magnitudes from Flower (1996). The bolometric correction is at a minimum for $\mathrm{A}$ stars and does not influence the luminosity calculation significantly. The effective temperatures were taken from Sect. 4.1. However, there is no homogeneous source of $V$ magnitudes that includes all our targets. We therefore calculated the averages from the magnitudes published by Kharchenko (2001) and Henden et al. (2016), and transformed the Gaia DR2 $G$ magnitudes according to the calibration by Arenou et al. (2018). Within these three data sets, we found no outliers larger than $0.015 \mathrm{mag}$. For the error calculation, we took a full error propagation of the individual errors into account.

Lindegren et al. (2018) found that the Gaia parallaxes require a zero-point offset of 0.03 mas, although, the offset is dependent on the colour, magnitude and position on the sky (Lindegren et al. 2018; Zinn et al. 2019). Murphy et al. (2019) have experimented with applying the suggested offset of 0.03 mas, but found that this correction results in unrealistically low luminosities for A and F stars. They deduce that the zero-point offset may be smaller for bluer stars. Since there is no consensus on the exact correction, we follow Murphy et al. (2019) and do not apply any zero-point offset in our analyses. We also note that Arenou et al. (2018) discourage users of applying the offset to individual parallaxes.

The stellar parameters with their respective uncertainties are listed in Table 2. If we compare the absolute magnitudes derived from the Hipparcos and Gaia DR2 data sets using the identical apparent magnitude, reddening and Bolometric correction, we find only three targets for which the deviation is larger than $3 \sigma$, specifically $v$ Pic, BX Ind, and $\theta$ Gru. This proves that the Gaia DR2 data have no significant discrepancies (in a statistical sense) when compared to the Hipparcos ones. We also note that these stars are bright which may explain the deviation. In addition, $\theta$ Gru was identified as a binary (see A1), which may apply for the other two as well.

In Fig. 2 we plot our stars in the HR diagram using Gaia luminosities and $T_{\text {eff }}$ determined in this work using the SED method. Due to binarity, large error bars in their Gaia measurements or missing parallaxes the following stars have been omitted: $\theta$ Ret $(\mathrm{V}=6.05)$, HD $223991(\mathrm{~V}=6.35)$, HD $216941(\mathrm{~V}=9.49)$, and HD $210767(\mathrm{~V}=7.78)$.

\section{UNDERSTANDING THE EXCITATION MECHANISM OF $\delta$ SCT STARS USING AM STARS}

In the absence of mixing, which is usually related to rotation and efficient convection in the outer envelopes, atomic diffusion, which includes radiative levitation and gravitational settling, plays an important role in A stars. As a result, Am stars display underabundances of Sc and $\mathrm{Ca}$ and overabundances of $\mathrm{Ba}, \mathrm{Sr}$ and $\mathrm{Y}$, among other elements, in their photospheres (Preston 1974).

Traditionally, the excitation mechanism associated with $\delta$ Sct pulsations is the $\kappa$-mechanism acting in the He II ionisation layer (Cox 1963). However, observations (Antoci et al. 2011; Murphy et al. 2019) clearly indicate that this mechanism alone is insufficient. This is especially the case for the chemically peculiar Am stars where He is expected to be depleted from the He II ionisation layer where the $\kappa$ mechanism operates. Even when including atomic diffusion (Turcotte et al. 2000), models in only a very narrow region in the instability strip were found to have unstable modes, 


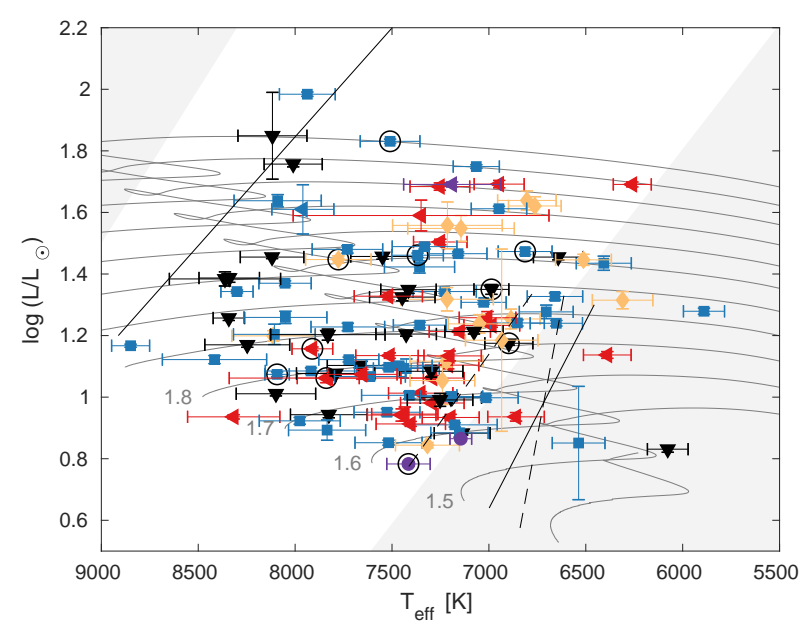

Figure 2. Intermediate-mass $A$ and $F$ stars observed by TESS in sectors 1 and 2 in the HR diagram. Non-variable stars are shown as black downward facing triangles, $\delta$ Sct stars as blue squares, $\gamma$ Dor stars as violet circles, hybrids or suspected hybrids in yellow diamonds, and HADS stars as left-facing red triangles. Stars marked with a black circle are known binary stars. The blue and red edges of the $\delta$ Sct instability domain (marked by the straight, continuous black lines) are taken from Pamyatnykh (2000) and Dupret et al. (2005), respectively. The blue edges from Pamyatnykh (2000) and Dupret et al. (2005) more or less overlap. The $\gamma$ Dor instability strip marked by the dashed lines is from Dupret et al. (2005). In addition, we highlight in white the $\delta$ Sct instability strip found empirically by Murphy et al. (2019) within which $>20 \%$ of the population pulsates (peaking at $70 \%$ ). The evolutionary tracks were computed with the Warsaw-New Jersey models (Dziembowski 1977; Pamyatnykh et al. 1998; Pamyatnykh 1999) using no rotation and solar metallicity. The evolutionary tracks, labeled with masses expressed in $\boldsymbol{M}_{\odot}$, are only displayed to guide the eye.

which is clearly contradicted by observations (e.g., Smalley et al. 2017).

Here we investigate to what extent the pulsational stability changes when helium is depleted from the outer layers. More specifically we have used the same approach as described in Balmforth et al. (2001) to model the helium abundance in the outer layers, to mimic atomic diffusion. For our models with a chemically homogenous envelope, the helium abundance by mass is $\mathrm{Y}=0.28$, whereas for our depleted models the helium abundance was decreased to $\mathrm{Y}=$ 0.15 in the outer layers, extending down beneath the He II ionisation layer.

In Fig. 3 and Fig. 4 we show results of our stability computations, in which we include the pulsationally perturbed radiative ( $\kappa$-mechanism) and convective heat fluxes as well as the perturbed momentum flux (turbulent pressure). These computations were obtained from models following the procedures described in detail in Houdek et al. (1999) and Balmforth et al. (2001). These models adopt the time-dependent non-local convection formulation by Gough (1977a,b). In addition to the mixing length parameter, $\alpha_{\mathrm{MLT}}$, this formulation uses three additional non-local convection parameters, $a, b$ and $c$. Here we adopt the values $a^{2}=b^{2}=c^{2}=950$, which allowed Antoci et al. (2014) to reproduce roughly
85 per cent of the observed frequency range of HD 187547 (a pulsating Am star) and Balmforth et al. (2001) to reproduce oscillations in roAp stars.

In Fig. 3 we plot the theoretical instability region of our 'mixed models' (i.e., He non-depleted) and Fig. 4 illustrates the instability domain of our depleted models. The asterisks show the locations of the computed models in the HR diagram and the number of excited radial orders found for that particular model is indicated. The contour levels in this plot delineate the number of consecutive excited radial-order modes. The redder the area, the larger number of modes to be excited. Yellow indicates no excitation at all. We note that in both cases, i.e. in the homogenous and the depleted envelope models, there seems to be a maximum in the number of excited radial orders at an effective temperature of $7500 \mathrm{~K}$. Interestingly, the largest number of unstable modes roughly corresponds with the temperature where Murphy et al. (2019) found the largest fraction of pulsating stars, although slightly shifted in luminosity.

In Fig. 5, panels (a1) and (b1), we show the accumulated work integrals for a radial mode with $n=6$ and a mode with $n=15$, respectively, in our chemically homogeneous and Hedepleted models. Regions where the accumulated work integral increases towards the surface of the star contribute to the driving of the mode while regions where it decreases contribute to the damping. Panels (a2) and (b2) further show the individual contributions to the total accumulated work integral of the gas (dashed line) and of the turbulent pressure (solid line). For both the homogenous (Fig. 3) and the He-depleted envelope models (Fig. 4), the turbulent pressure contributes crucially to mode instability.

In this paper, we do not aim to fully reproduce the instability domain of $\delta$ Sct stars, but to show that diffusion, especially of He, plays an important role in driving pulsations in $\delta$ Sct stars, and that turbulent pressure, acting in the $\mathrm{H}$ ionisation zone is a significant driving agent. Therefore, excitation by turbulent pressure offers a solution to the long-standing question of the driving mechanism in pulsating Am stars. In Fig. 6 we plot all Am stars from Renson \& Manfroid (2009) that were observed by TESS in sectors 1 and 2. We distinguish between constant (black triangles) and pulsating Am (blue) stars, and mark known binaries with black rings. We find good agreement between the observed Am stars and the predicted instability region when turbulent pressure is included. Our findings also agree well with earlier ground-based observations, showing that pulsating Am stars tend to be found in the cooler part of the instability region (Smalley et al. 2017).

To conclude this section, we show that even in the case where $\mathrm{He}$ is depleted from the outer layers, excitation of pulsations is still possible and matches the observed distribution of known pulsating Am stars observed by TESS. In addition, we see an increase in the number of unstable radial orders and magnitude of the linear growth rates in stars with $T_{\text {eff }} \simeq 7500 \mathrm{~K}$. Here we demonstrate the potential of how TESS observations of many Am stars will improve our understanding of pulsation driving in intermediate-mass stars. Before drawing any final conclusions, however, we need to perform in-depth analyses and to vary parameters in our computations, e.g., metallicity, the mixing length and the non-local convection parameters $a, b$, and $c$. In addition, we 
will also explore the excitation at different helium depletion values. This will be done in a future paper.

\section{EXAMPLES OF VARIABILITY}

As TESS is observing almost the entire sky, we have the unprecedented opportunity to obtain continuous, highprecision light curves of a variety of pulsating stars as described in Section 1. The 2-min TESS sample in sectors 1 and 2 presented in this work includes some prototypes, i.e., SX Phe and $\gamma$ Dor. In this section, we demonstrate the power of TESS data for a variety of different pulsator classes amongst $\mathrm{A}$ and $\mathrm{F}$ stars and the potential of future asteroseismic modelling. The stars shown here were chosen either because they are eponymous for their classes (SX Phe and $\gamma$ Dor), very bright ( $\alpha$ Pic) or to cover the entire variety of the stars studied here: $\delta$ Sct, $\gamma$ Dor, $\lambda$ Boo, HADS, pre main-sequence, eclipsing binary stars.

\subsection{TIC 229059574 = HD 210111}

TIC 229059574 (HD 210111), is a $\lambda$ Boo star in a doublelined spectroscopic binary (SB2) system (Paunzen et al. 2012). SB2 line profiles were seen in all 24 high-resolution spectra taken by Paunzen et al. (2012) with UVES, from which the authors concluded that this is a pair of equalmass stars with both stars having $T_{\text {eff }} \approx 7400 \mathrm{~K}, \log g=$ $3.8,[\mathrm{Fe} / \mathrm{H}]=-1.0$ dex and $v \sin i=30 \mathrm{~km} \mathrm{~s}^{-1}$. By computing composite spectra with various atmospheric parameters, they also concluded that neither component has a solar metallicity, from which we infer that they are both $\lambda$ Boo stars.

Paunzen et al. (1994) first discovered the $\delta$ Sct variability of HD 210111, and Breger et al. (2006) carried out the most extensive analysis of its variability with a multi-site ground-based campaign over a 30-d period. The TESS light curve is of similar duration, but almost uninterrupted and of space-telescope quality.

The TESS light curve from sector 1 shows HD 210111 to be highly multi-periodic with frequencies in the range $10-40 \mathrm{~d}^{-1}$ and amplitudes of order 100-1000 ppm (Fig. 7). The highest peak lies at $30.2 \mathrm{~d}^{-1}$, consistent with the nonradial pulsation with a 49 -min period identified by Bohlender et al. (1999). Many of the 18 oscillation frequencies reported by Breger et al. (2006) are present in the TESS data, but the amplitudes are significantly different. This may be due to the different passbands, although amplitude variability is not uncommon in $\delta$ Sct stars (Lenz et al. 2008; Barceló Forteza et al. 2015; Bowman et al. 2016). In the TESS passband, HD 210111 has 59 peaks with amplitudes exceeding $200 \mathrm{ppm}$, where our analysis is terminated because of the high density of peaks.

Visual inspection of the amplitude spectrum of the light curve (Fig. 7) suggests there may be a common frequency spacing between $\sim 2$ and $2.5 \mathrm{~d}^{-1}$. When using all 59 peaks above 200 ppm, a histogram of frequency differences showed no outstanding features compared to a randomly generated set of frequencies covering the same frequency range. However, a subset of the strongest peaks (with amplitudes above $500 \mathrm{ppm}$ ) showed an excess of peaks spaced by $\sim 2.2 \mathrm{~d}^{-1}$. Common spacings can sometimes be attributed to rotational

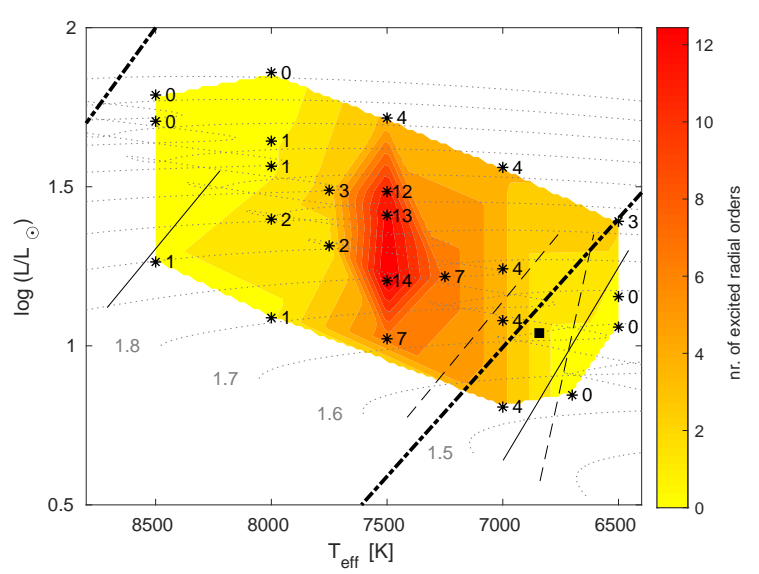

Figure 3. Theoretical instability strip from models including time-dependent non-local convection treatment (Section 5). The asterisks indicate the location of our theoretical models and the adjacent number indicates the number of excited radial orders. In straight, continuous black lines we mark the theoretical blue and red edges of the $\delta$ Sct instability domain from Pamyatnykh (2000) and Dupret et al. (2005), respectively. The blue edges from Pamyatnykh (2000) and Dupret et al. (2005) are more or less overlapping. The black square is the red edge computed by Houdek (2000). For convenience we also indicate the $\gamma$ Dor instability strip in dashed lines (Dupret et al. 2005). In addition, we also depict the empirical $\delta$ Sct instability strip boundaries from Murphy et al. (2019) at which the pulsator fraction is $20 \%$ (dashed-dotted line). The evolutionary tracks were computed with the WarsawNew Jersey models (Dziembowski 1977; Pamyatnykh et al. 1998; Pamyatnykh 1999) using no rotation and solar metallicity. The evolutionary tracks are only displayed to guide the eye.

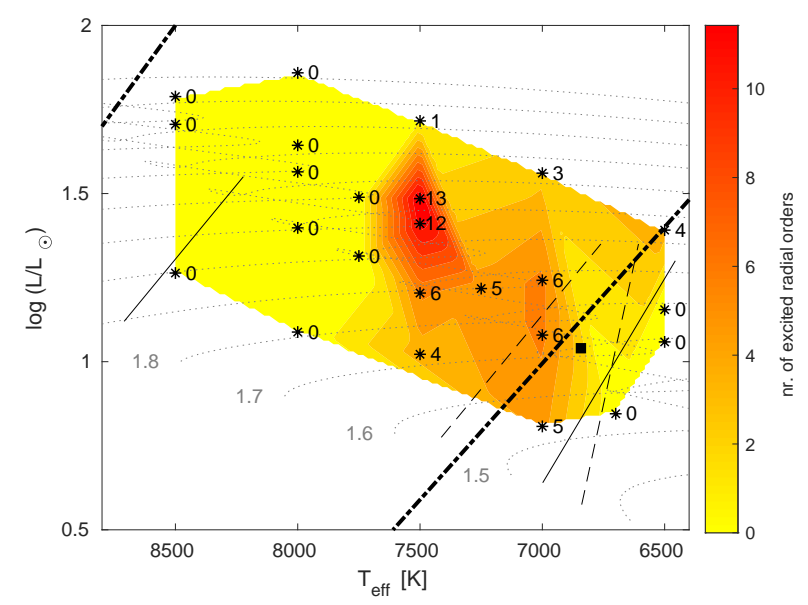

Figure 4. Theoretical instability strip with depleted helium in the outer layers mimicking helium settling. Models include timedependent non-local convection treatment (Section 5). The asterisks indicate the location of our theoretical models and the adjacent number indicates the number of excited radial orders. The instability domains and evolutionary tracks are the same as in Fig. 3. 

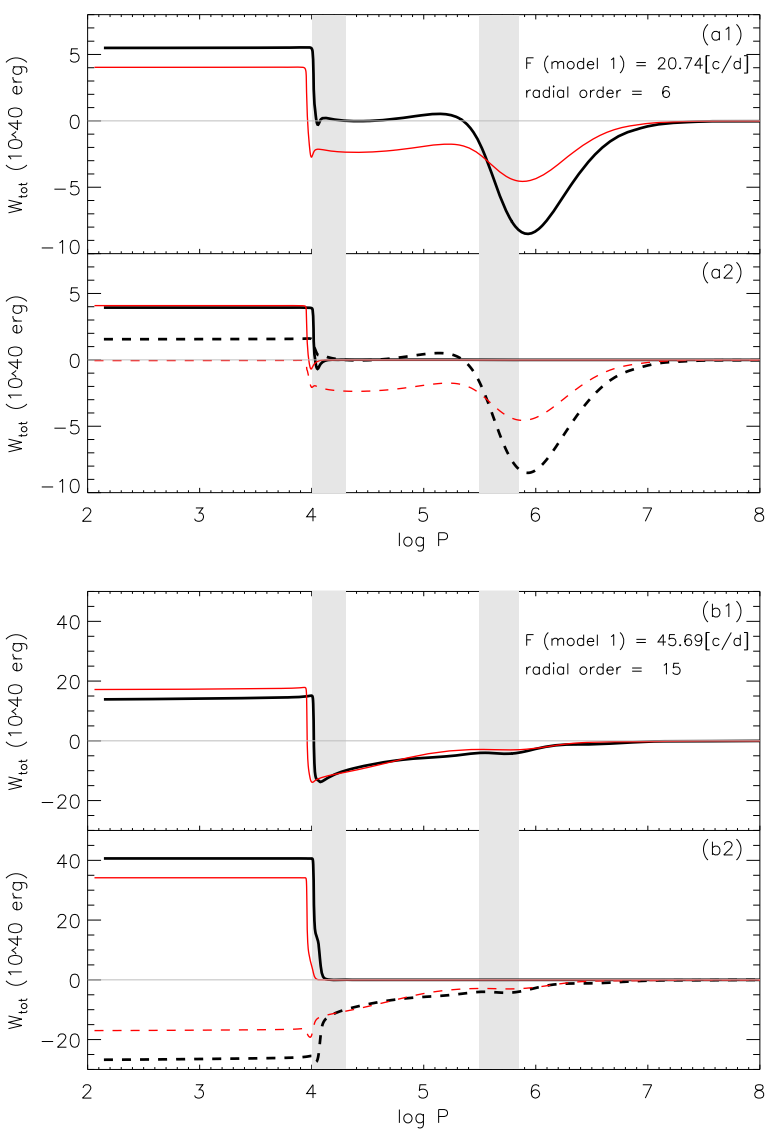

Figure 5. Accumulated work integrals (solid curves in panels (a1) and (b1)) as a function of the total pressure for two different radial orders, $n=6$ and $n=15$, for the homogeneous model (black) and the He-depleted model (red in the online and grey in the printed version). The modes are unstable whenever the accumulated work integrals are positive towards the surface of the star, as displayed in (a1) and (b1). In panels (a2) and (b2) we show the contributions of the gas pressure (dashed line) and the turbulent pressure (solid line). The grey region at $\log P=6$ indicates theHE II, and at $\log P=4$ the $\mathrm{H} \mathrm{I}$ and $\mathrm{HE} \mathrm{I}$ ionization zones. From panels (a2) and (b2) it is evident that turbulent pressure in the $\mathrm{H} \mathrm{I}$ and $\mathrm{HE} \mathrm{I}$ ionisation zones plays a significant role in the excitation of both modes. Specifically in the case of $n=15$, it is the only driving agent, while the gas pressure (responsible for the $\kappa$-mechanism) is damping pulsations in the HE II ionisation zone. The model parameters are in both cases the following: $\mathrm{M}=2.3 \mathrm{M}_{\odot}, T_{\text {eff }}=7500 \mathrm{~K}, \log L=1.48 \mathrm{~L}_{\odot}$ and correspond to the model with 13 unstable radial orders in Fig. 3 and 12 in Fig. 4, respectively.

splittings, or to the large frequency separation $\Delta v$, which is the frequency spacing between consecutive radial orders ${ }^{7}$. The $v \sin i$ of these stars is low, at about $30 \mathrm{~km} \mathrm{~s}^{-1}$, so it is unlikely that the rotational splitting of one or both stars is being observed, unless the inclination $i$ happens to be low. We therefore find it more plausible that the identified spacing corresponds to the large frequency separation or half this

7 The exact spacing depends on the radial orders and can only be directly related to the Sun or Sun-like stars in case high radial order modes are observed.

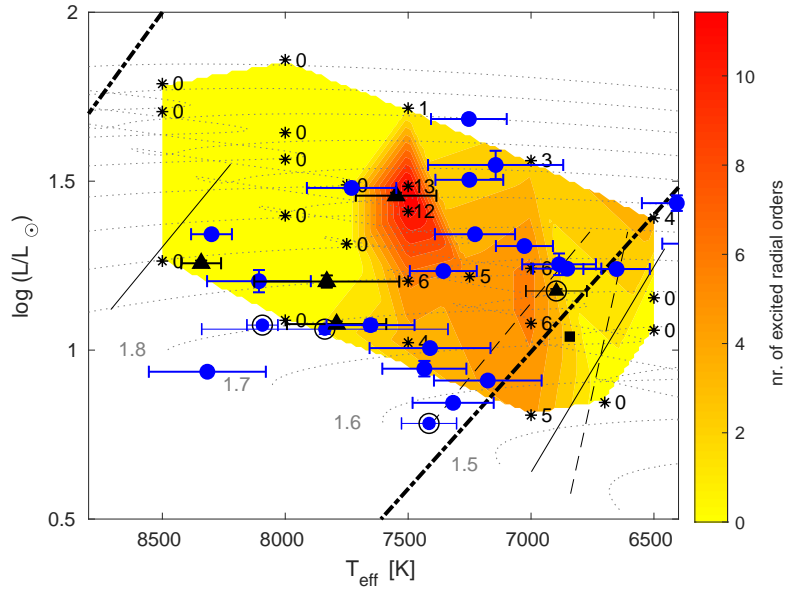

Figure 6. Comparison between the observed TESS Am stars and the theoretical instability strip including He depletion in the outer envelope. All stars are identified to be Am stars based on the Renson catalogue (Renson \& Manfroid 2009). The blue circles depict the pulsating Am stars; the black triangles identify constant Am stars. Known binaries (see Table 1) are marked by black rings. The square, asterisks and all lines have the same meaning as in Fig. 3.
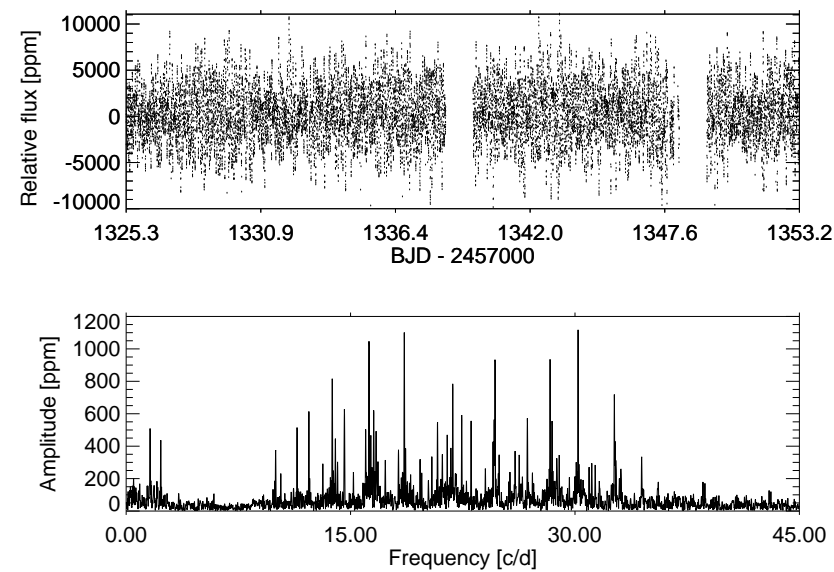

Figure 7. TESS light curve (top) and amplitude transform (bottom) of the $\lambda$ Boo star HD 210111.

value. A more accurate assessment using a Gaia luminosity is prevented by the SB2 nature of the target. It is also not clear whether solar-metallicity tracks should be used. The reported $[\mathrm{Fe} / \mathrm{H}]$ is -1 dex, but in $\lambda$ Boo stars the low metallicity might be a surface phenomenon only. Asteroseismology is one of the tools that can be used to probe the global stellar metallicity, but HD 210111 is perhaps too complex a target to be a good starting point because of it being a binary of two similar stars. At this stage it is unclear whether only one or both components are pulsating. A more detailed analysis is beyond the scope of this paper. 


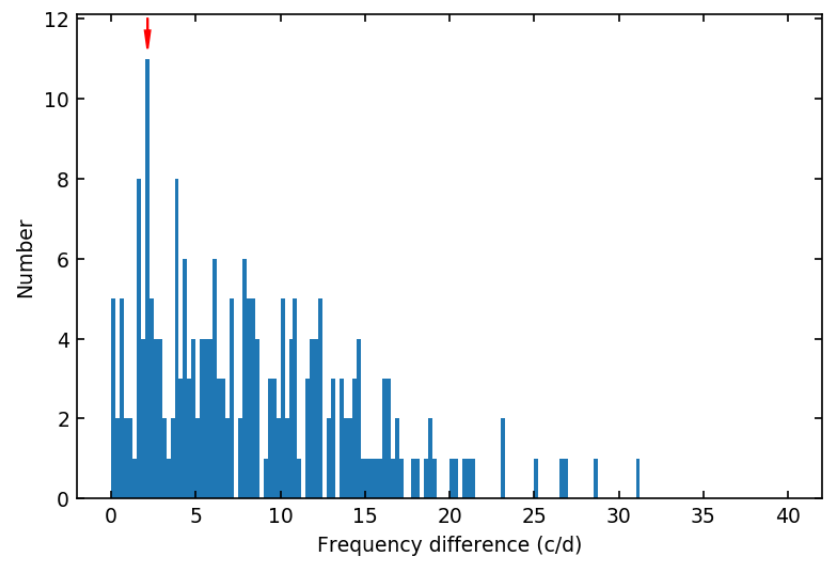

Figure 8. A histogram of the frequency differences from the peaks extracted from HD 210111 that have amplitudes exceeding $500 \mathrm{ppm}$. The peak at $2.2 \mathrm{~d}^{-1}$ is marked with a red arrow. Integer multiples of this peak $\left(4.4,6.6, \ldots \mathrm{d}^{-1}\right)$ are also expected.

\subsection{TIC $167602316=\alpha$ Pictoris}

$\alpha$ Pictoris is the brightest star in the sample presented here $(V=3.3)$ and was not known to be a pulsating star prior to TESS observations. This makes it one of the brightest known $\delta$ Sct stars. This star has $T_{\text {eff }}=7550 \pm 35 \mathrm{~K}$ (Zorec \& Royer 2012a), a Hipparcos luminosity of $\log \left(\mathrm{L}_{/} \mathrm{L}_{\odot}\right)=1.47 \pm 0.14$ and is rapidly rotating with $v \sin i=206 \mathrm{~km} \mathrm{~s}^{-1}$ (Zorec \& Royer 2012a).

$\alpha$ Pic has a rich pulsation spectrum (Fig. 9), with 39 significant peaks after 2 sectors of observations. From the lower panel of Fig. 9 it can be clearly seen that many additional peaks are observed in the low-frequency region that are consistent either with $\mathrm{g}$ and/or Rossby modes. In the high frequency range we detect a spacing that is consistent with the large frequency separation of about $2.7 \mathrm{~d}^{-1}$ and fits well the stellar parameters given Table 2 .

Based on the known stellar parameters, the highest peak at $f_{1}=5.74 \mathrm{~d}^{-1}$ could be the fundamental radial mode. The frequency $f_{3}$ is close to $2 f_{1}$, which may imply that this frequency is the second harmonic, however it is not an exact match (the difference is $0.017 \mathrm{~d}^{-1}$ ), which is similar to the resolution. This issue will be resolved with one year of data, as this star is in the continuous TESS viewing zone. No other obvious combination frequencies were detected.

\subsection{TIC 224285325 = SX Phe}

SX Phe is a remarkable HADS star, whose strongest peak exceeds $100 \mathrm{ppt}$. The light curve is highly asymmetric, with minima of a consistent depth, but maxima spanning a wide range in brightness (Fig. 10). Such light curves are common among high-amplitude SX Phe stars dominated by one or two mode frequencies and their combinations (e.g., the $\mathrm{Ke}$ pler SX Phe star KIC 11754974; Murphy et al. 2013). The shapes of the light curves arise from specific phase relationships between parent pulsation modes and their combination frequencies (Kurtz et al. 2015).

The amplitude spectrum of the light curve of SX Phe
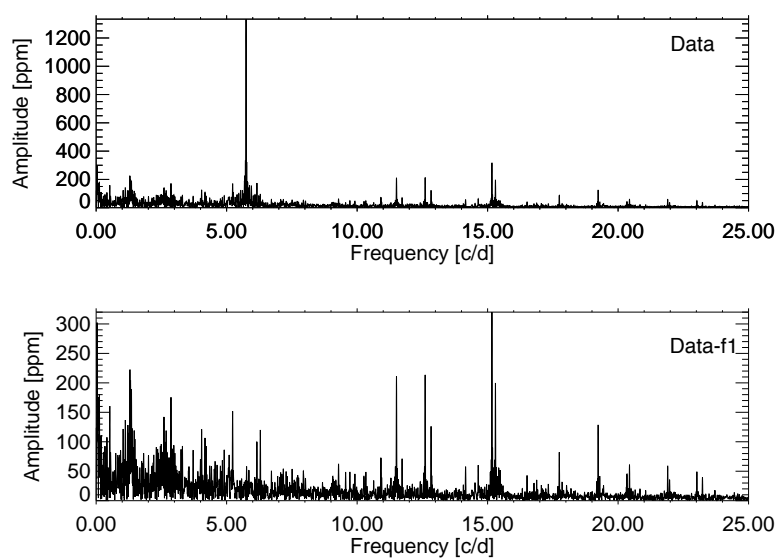

Figure 9. Amplitude spectra of the original data of $\alpha$ Pic (top) and after pre-whitening the dominant mode (bottom). This star is in the TESS continuous viewing zone on the Southern hemisphere which makes it a promising target for further study.
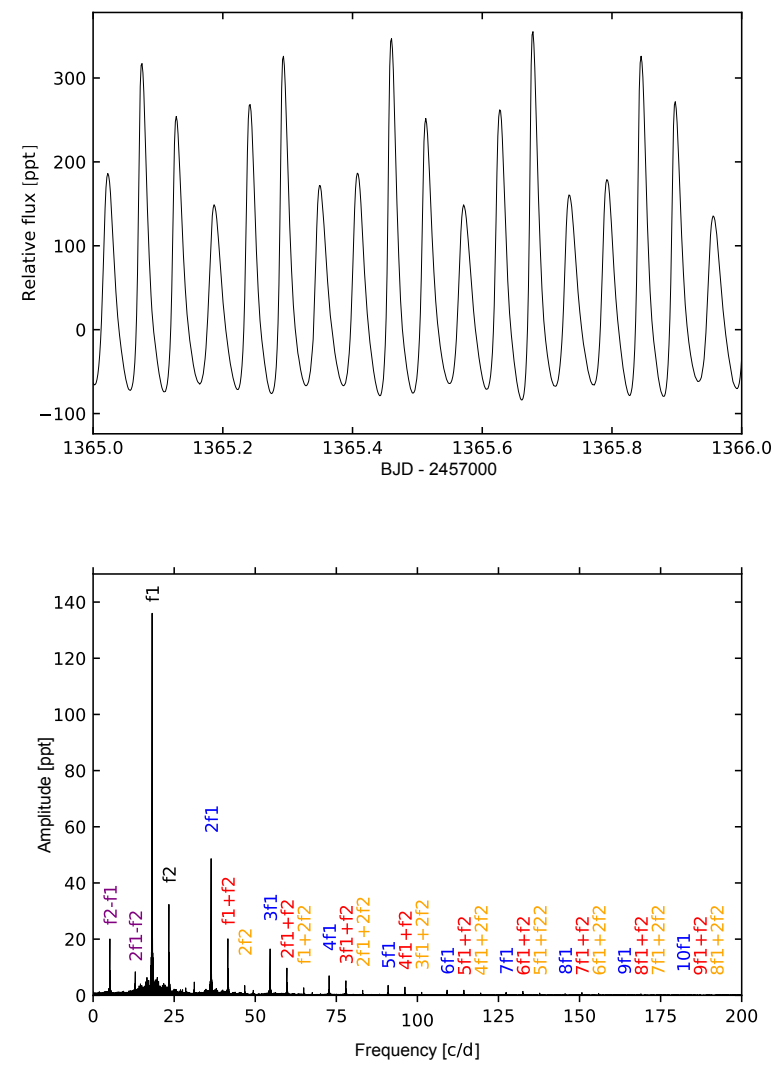

Figure 10. A 1-d extract of the TESS light curve of SX Phe (top). Amplitude spectrum of the entire TESS light curve of SX Phe (bottom). The two strong independent mode frequencies are labelled along with combination frequencies belonging to three distinct families, and two other combinations at lower frequencies. 
Table 5. The list of extracted significant frequencies of SX Phe from TESS Sector 1 and 2 data.

\begin{tabular}{llcc}
\hline$f_{n}$ & $\begin{array}{l}\text { Frequency } \\
{\left[\mathrm{d}^{-1}\right]}\end{array}$ & $\begin{array}{c}\text { Amplitude } \\
{[\mathrm{ppt}]} \\
\pm 0.040\end{array}$ & Combinations \\
\hline$f_{1}$ & $18.193565(6)$ & 136.284 & \\
$f_{2}$ & $23.37928(2)$ & 33.079 & \\
$f_{3}$ & $36.38712(2)$ & 48.757 & $2 f_{1}$ \\
$f_{4}$ & $41.57284(4)$ & 20.286 & $f_{1}+f_{2}$ \\
$f_{5}$ & $5.185718(4)$ & 20.133 & $f_{2}-f_{1}$ \\
$f_{6}$ & $54.58069(5)$ & 16.653 & $3 f_{1}$ \\
$f_{7}$ & $13.00784(8)$ & 10.237 & $2 f_{1}-f_{2}$ \\
$f_{8}$ & 59.766413 & 9.801 & $2 f_{1}+f_{2}$ \\
$f_{9}$ & $72.7742(1)$ & 6.939 & $4 f_{1}$ \\
$f_{10}$ & $77.9599(2)$ & 5.047 & $3 f_{1}+f_{2}$ \\
$f_{11}$ & $31.2014(2)$ & 4.948 & $3 f_{1}-f_{2}$ \\
$f_{12}$ & $46.7585(2)$ & 3.572 & $2 f_{2}$ \\
$f_{13}$ & $90.9678(2)$ & 3.527 & $5 f_{1}$ \\
$f_{14}$ & $96.1535(3)$ & 2.893 & $4 f_{1}+f_{2}$ \\
$f_{15}$ & $22.6303(3)$ & 2.774 & \\
$f_{16}$ & $28.5650(3)$ & 2.692 & $2 f_{2}-f_{1}$ \\
$f_{17}$ & $64.9521(3)$ & 2.632 & $f_{1}+2 f_{2}$ \\
$f_{18}$ & $27.5864(4)$ & 2.074 & \\
$f_{19}$ & $114.3471(4)$ & 1.931 & $5 f_{1}+f_{2}$ \\
$f_{20}$ & $109.1613(4)$ & 1.831 & $6 f_{1}$ \\
$f_{21}$ & 49.3950042 & 1.798 & \\
$f_{22}$ & $83.1456(5)$ & 1.738 & $2 f_{1}+2 f_{2}$ \\
$f_{23}$ & $132.5406(5)$ & 1.495 & $6 f_{1}+f_{2}$ \\
$f_{24}$ & $40.823(3)$ & 1.198 & $f_{1}+f_{2} 0$ \\
$f_{25}$ & $127.355(1)$ & 1.118 & $7 f_{1}$ \\
$f_{26}$ & $17.2645(7)$ & 1.105 & \\
$f_{27}$ & $45.7806(7)$ & 1.072 & \\
\hline & & & \\
\hline
\end{tabular}

itself is indeed dominated by two parent radial modes and their many combination frequencies (Fig. 10). These combinations and their harmonics continue to high frequencies: even at $7 f_{1}\left(127 \mathrm{~d}^{-1}\right)$, the pulsation amplitude is over $1 \mathrm{ppt}$. We extracted frequencies down to an amplitude of 1 ppt. Five other apparently independent peaks exist with amplitudes above $1 \mathrm{ppt}$. Although it is possible to assign combinations to some of them, the combinations are not simple (i.e. they do not have low coefficients), so we conclude that some of these peaks could be independent mode frequencies and speculate that resonance may play an important role. A frequency list is provided in Table 5 .

The strongest mode corresponds to the frequency $f_{1}=$ 18.193565(6) $\mathrm{d}^{-1}$, which is high for the fundamental radial mode but rather typical for SX Phe-type stars (e.g., McNamara 2011; Ziaali et al. 2019). The frequency ratio of $f_{1} / f_{2}$ is $0.778192(6)$, is consistent with the identification of $f_{1}$ as the fundamental radial mode and $f_{2}$ as the first radial overtone. Normal $\delta$ Sct stars have values around 0.772 , but SX Phe itself has a rather high value of $0.778192(6)$, owing mostly to its low metallicity. Petersen \& ChristensenDalsgaard (1996) showed that higher values of this frequency ratio correlate well with lower metallicity, and SX Phe itself has a low metallicity, with $Z \approx 0.001$. In addition, Pigulski (2014) shows that other SX Phe stars can also have such a high ratio.

Using the Warsaw-New Jersey evolutionary code (e.g., Pamyatnykh et al. 1998; Pamyatnykh 1999) and the nona- diabatic pulsational code of Dziembowski (1977), we computed models for SX Phe with the aim of fitting $f_{1}$ and $f_{2}$ as the radial fundamental and first overtone modes, respectively, using the so-called Petersen diagram (e.g., Petersen \& Christensen-Dalsgaard 1996). We searched a wide range of effective temperatures ranging from $7200 \mathrm{~K}$ to $8700 \mathrm{~K}$. The OPAL opacity tables and the solar element mixture of Asplund et al. (2009) were adopted. We considered different metallicity values, $Z$, and initial hydrogen abundance, $X_{0}$, in the ranges $(0.001,0.002)$ and $(0.66,0.74)$, respectively. The value of the mixing length parameter, $\alpha_{\text {MLT }}=1.5$ and overshooting from the convective core was not included. We note that, at the metallicities and effective temperatures specified above, the choice of the $\alpha_{\text {MLT }}$ does not have a high impact on the exact values of the mode frequencies. Our pulsations models, confirm that the observed frequencies $f_{1}$ and $f_{2}$ can only correspond to the radial fundamental and first overtone modes, respectively. Thus, for further seismic modelling we will only consider these radial orders.

As a start, we used the model parameters $\mathrm{M}=1.0 \mathrm{M}_{\odot}$, $Z=0.001, X_{0}=0.70$ and an age of roughly $3.9 \mathrm{~Gy}^{8}$ as given by Petersen \& Christensen-Dalsgaard (1996). Our best seismic model for these input values has an effective temperature of $T_{\text {eff }}=7660 \mathrm{~K}$, a luminosity $\log \mathrm{L} / \mathrm{L}_{\odot}=0.811$ and a frequency ratio of the radial fundamental mode to the first overtone of $f_{1} / f_{2}=0.780014$. The observed counterparts are $\log \mathrm{L} / \mathrm{L}_{\odot}=0.842 \pm 0.09$ and a frequency ratio of $0.778192(6)$. The theoretical values for the radial fundamental and the first radial overtone are $18.193565 \mathrm{~d}^{-1}$ and $f_{2}=23.324654$ $\mathrm{d}^{-1}$, respectively. Both radial modes are unstable (excited). However, as one can see, the difference between the theoretical and observed value of $f_{1} / f_{2}$ is 0.001822 and is above the numerical accuracy which is around the fifth decimal place, making it clearly significant. In the next step, we varied the metallicity and found a model with $Z=0.0014$ and a mass $M=1.15 M_{\odot}$, which fits better the observed frequency ratio. The model has the following parameters: $T_{\text {eff }}=8270$, $\log \mathrm{L} / \mathrm{L}_{\odot}=0.984$, an age of $\sim 2.5$ Gy and a frequency ratio of $f_{1} / f_{2}=0.77841$. The individual radial modes frequencies are $f_{1}=18.19357 \mathrm{~d}^{-1}$ and $f_{2}=23.37268 \mathrm{~d}^{-1}$. This model gives a better fit but still there is a room for improvement, especially because the radial fundamental and the first overtone in this model are both stable.

As a next step, we changed the initial abundance of hydrogen. The model reproducing the observed frequency ratio up to the fifth decimal place has the following parameters: $M=1.05 M_{\odot}, X_{0}=0.67, Z=0.002, T_{\text {eff }}=7760 \mathrm{~K}$, $\log \mathrm{L} / \mathrm{L}_{\odot}=0.844$, an age of $2.8 \mathrm{~Gy}$ and it was interpolated to the dominant observed frequency $f_{1}=18.193565 \mathrm{~d}^{-1}$. The value of the second frequency is $23.37976 \mathrm{~d}^{-1}$, and differs by only $0.00048 \mathrm{~d}^{-1}$ from the observed value. Thus, the theoretical value of the frequency ratio is 0.77818 , whereas the observed value is 0.77819 . Taking into account the numerical accuracy, which is not better than five decimal places as specified above, we can conclude that this model reproduces the observed frequencies of the two radial modes of SX Phet well. In addition, this model also predicts instability (excita-

8 Given that SX Phe is consistent with the scenario of a blue straggler, the age in this context represents the time since its formation through mass transfer or a merger of two stars. 


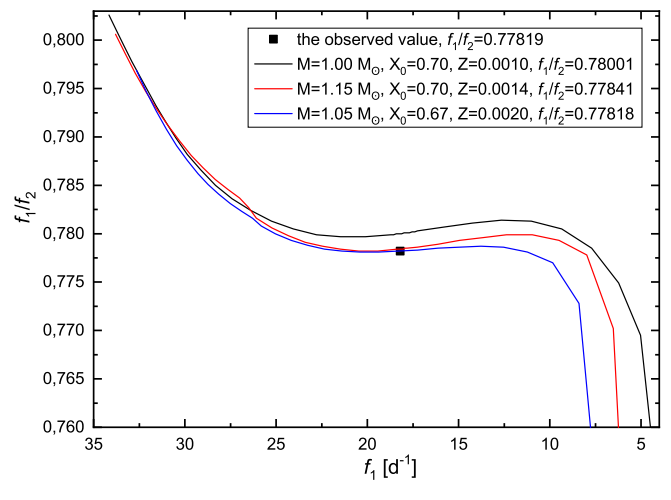

Figure 11. Petersen diagram illustrating the frequency ratio of the first radial overtone to the radial fundamental as a function of the radial fundamental mode for SX Phe. The model reproducing the observations best is depicted in blue. We refer the reader to the text for further details.

tion) of both radial modes, the radial fundamental and first overtone. In Fig. 11, we show the evolution of the frequency ratio of the first radial overtone to the radial fundamental as a function of the radial fundamental mode (the Petersen diagram) for the three seismic models described above. The observed value is marked as an open square.

The lower hydrogen abundance (higher helium abundance) is not unlikely because, as other SX Phoenicis variables, SX Phe itself can be a blue straggler. Such objects are presumably formed by the merger of two stars or by interactions in a binary system (e.g., McNamara 2011; Nemec et al. 2017). As a consequence, these stars may have enhanced helium abundance. This is also consistent with the models fitting KIC 11145123, which is also a suspected blue straggler (Kurtz et al. 2014). In addition, the model luminosity also agrees with the Gaia luminosity and the model effective temperature is within $2 \sigma$ of the spectroscopically derived value $\left(T_{\mathrm{eff}}=7500 \pm 150 \mathrm{~K}\right.$, and $3.7 \sigma$ within the photometrical $\left(T_{\text {eff }}=7210 \pm 150 \mathrm{~K}\right)$. Measured differences in effective temperature are to be expected, because as noted by Kim et al. (1993), the effective temperature of a star displaying large amplitudes of pulsations depends on the pulsation phase at which the observations were made. In the case of SX Phe the spectroscopic $T_{\text {eff }}$ was determined based on one spectrum taken at a single unknown pulsation phase.

\subsection{TIC $49677785=$ HD 220687}

HD 220687 (TIC 49677785) is a known eclipsing binary system hosting a pulsating primary (Pigulski \& Michalska 2007). Recently, HD 220687 was observed for approximately $80 \mathrm{~d}$ by the K2 mission (Howell et al. 2014). From these K2 data and estimates of the primary's surface gravity and effective temperature from RAVE spectroscopy, Lee et al. (2019) obtained a binary model with the Wilson Devinney Code, albeit without an estimate for the mass ratio from radial velocities. After subtracting their binary light curve solution, Lee et al. (2019) found 35 significant frequencies with a $\mathrm{S} / \mathrm{N} \geqslant 4$ using a noise window of the size $5 \mathrm{~d}^{-1}$ cen- tred around each peak; all of these frequencies were inferred to be $\delta$ Sct pulsations.

Since the K2 observations, HD 220687 was observed by the TESS mission in Sector 2 for $27.4 \mathrm{~d}$ (Fig. 12). Given the lack of an accurately constrained mass ratio and the presence of remaining harmonics after the binary model subtraction, we chose to not adopt the binary solution by Lee et al. (2019). Instead, we directly subtracted the orbital frequency $\left(f_{\text {orb }}=0.62725 \mathrm{~d}^{-1}\right)$ and all its significant harmonics up to $80 \mathrm{~d}^{-1}$ and then examined the remaining variance. From iterative pre-whitening pipeline we obtained 25 significant frequencies, 11 of which can be explained as harmonics or combination frequencies. We note that seven of the frequencies reported by Lee et al. (2019) are either harmonics of the orbital frequency, or can be explained as second-order combination frequencies with the orbital frequency. Finally, 12 of the 25 frequencies in the TESS light curve of HD 220687 are above the Kepler/K2 long-cadence Nyquist frequency of $24.49 \mathrm{~d}^{-1}$ which emphasizes the usefulness of short-cadence observations for studying $\delta$ Sct stars.

The TESS and K2 light curves of HD 220687 also offer the only opportunity we are aware of to determine an empirical estimate for the amplitude suppression factor of pulsation modes observed in the Kepler/K2 and TESS passbands. Since the TESS passband $(6000-10000 \AA)$ is redder than that of Kepler/K2 (4300-8300 $\AA$ ), the photometric pulsation mode amplitudes in early-type stars appear smaller in TESS photometry compared to bluer passbands since the wavelength range mainly probes the Rayleigh-Jeans tail, as opposed to the peak of the blackbody function. The ratio of pulsation mode amplitudes in the TESS and Kepler/K2 passbands for $\mathrm{A}$ and $\mathrm{F}$ stars was estimated to be of the order 50 per cent by Bowman et al. (2019), yet this value included many assumptions.

Here, we provide an empirical measurement of the ratio of pulsation mode amplitudes for the TESS and Kepler/K2 passbands using HD 220687 (TIC 49677785; EPIC 245932119). We downloaded the K2 target pixel files of HD 220687, created custom aperture masks and detrended light curve spanning approximately $80 \mathrm{~d}$ using the methodology described by Bowman et al. (2018). The calculation of an amplitude spectrum using a Discrete Fourier Transform (Deeming 1975; Kurtz 1985) allowed the determination of the amplitudes of pulsation modes and harmonics associated with the eclipses present in the light curve. We performed independent frequency extraction using the K2 and TESS light curves via iterative pre-whitening and corrected the observed amplitudes of each extracted peak for the amplitude suppression caused by the sampling frequency of each instrument using

$A=A_{0} \operatorname{sinc}\left(\frac{\pi}{N}\right)=A_{0} \operatorname{sinc}\left(\frac{\pi v}{v_{\text {samp }}}\right)$,

where $A$ and $A_{0}$ are the observed and corrected pulsation mode amplitudes, respectively, $N$ is the number of data points per pulsation cycle, $v$ is the pulsation mode frequency and $v_{\text {samp }}$ is the instrumental sampling frequency (Murphy 2014; Bowman et al. 2015).

Under the assumptions of negligible amplitude modulation of its pulsation modes (see e.g. Bowman et al. 2016) and negligible contamination in HD 220687, which is reasonable 

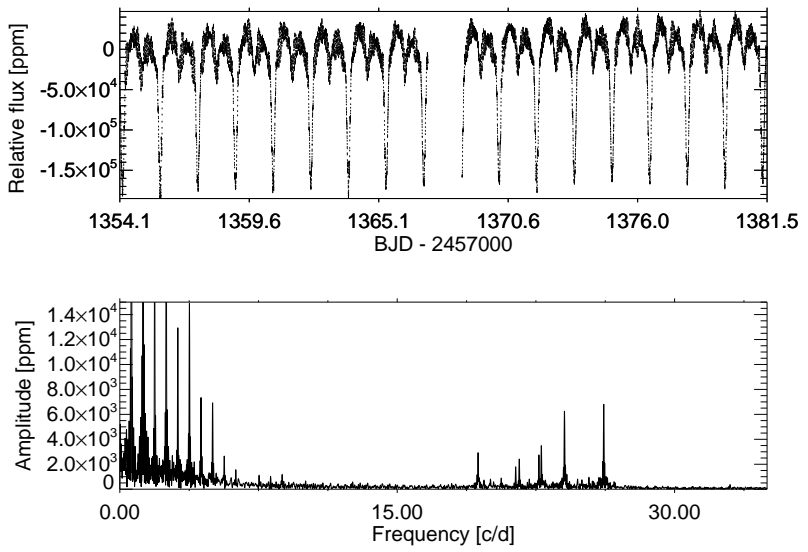

Figure 12. HD 220687. Upper panel: light curve. Lower panel: amplitude spectrum. The equally spaced peaks at low frequencies are due to the eclipses clearly visible in the light curve displayed in the upper panel.

given that HD 220687 is $V=9.6$ and no other nearby sources are apparent, the ratio of the corrected pulsation mode amplitudes gave an empirical estimate of the TESS/Kepler amplitude suppression factor. For the dominant, independent pulsation mode amplitudes in HD 220687, we measured an average ratio of their amplitudes in the TESS to Kepler/K2 passbands to be $74 \pm 1$ per cent. Thus, the reduction in pulsation mode amplitudes when observing $\mathrm{A}$ and $\mathrm{F}$ stars with TESS is non-negligible compared to bluer wavelength instruments such as Kepler/K2, but importantly this passband suppression does not prevent the detection of pulsation modes. On the other hand, the vast number of $\mathrm{A}$ and F stars observed in the 2-min TESS cadence is a major advantage for the study of high-frequency pulsations in $\delta$ Sct stars, given that the bias introduced by the 30-min Kepler cadence into the observed frequency distribution of these stars is much more significant (Murphy 2015; Bowman \& Kurtz 2018). We note that the suppression factor is not a constant but depends on the spectral type of the target star and to a lesser extent on the geometry of the pulsation modes (limb-darkening).

It is worth noting that $\delta$ Sct $/ \gamma$ Dor pulsators found in close binaries, are not only in detached systems but also in those that are undergoing or have experienced mass transfer (Guo et al. 2016, 2017). Among the 303 pulsating EB identified by Gaulme \& Guzik (2019, in prep.) in about 3000 Kepler EB stars, most are $\delta$ Sct and $\gamma$ Dor pulsators (264 out of $303,87 \%$ ). We expect that the TESS 2-min cadence data can yield about 300 eclipsing binaries per sector, and more than $1 / 10$ are expected to contain pulsating stars.

\subsection{TIC $150394126=$ HD 46190}

HD 46190 was observed in sectors 1 and 2 in the TESS mission (Fig. 13); its light curve has a total time base of $56 \mathrm{~d}$. Prior to TESS observations, this star was thought to be a young star with a spectral type of A0, but its pulsational variability was unknown. We include HD 46190 in this paper, as it is a potential pre-main-sequence $\delta$ Sct star.
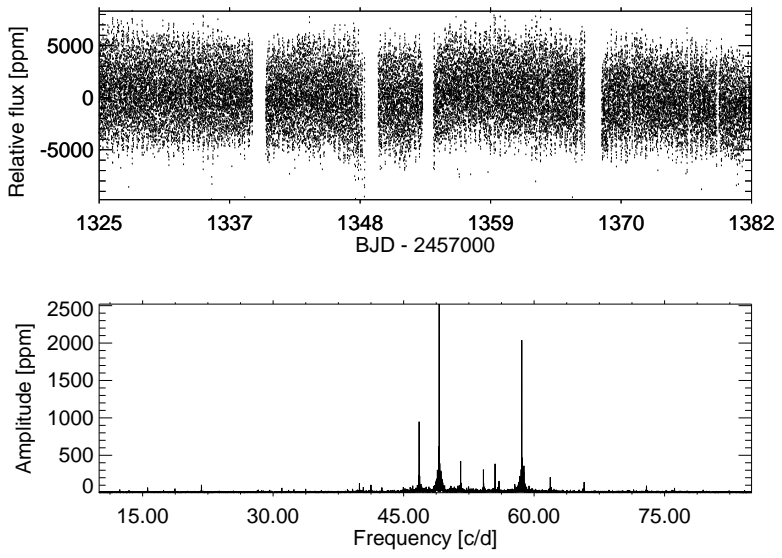

Figure 13. TESS light curve (top) and amplitude spectrum (bottom) of the young star HD 46190.

In total 65 frequencies between 15 and $76 \mathrm{~d}^{-1}$ were found to be statistically significant by both the KU Leuven and Aarhus ECHO pipelines. Many of these peaks can be explained by combination frequencies, depending on the number of parent modes assumed. However, some of these identified linear combinations are quite complex, and hence could also be accidental matches.

There are no estimates of fundamental parameters or binarity from spectroscopy in the literature, and only an approximate effective temperature of $8450 \pm 600 \mathrm{~K}$ in the catalogue of Oelkers et al. (2018) and Gaia DR2. Our determined effective temperature based on the SED method is $8850 \pm 100 \mathrm{~K}$ (Table 2). Sloan et al. (2004) report a debris disk and an infrared excess around HD 49160 using Spitzer spectroscopy, and identify this star as a Vega-like main-sequence star.

The highest-amplitude p-mode frequency in HD 46190 is at $49.09 \mathrm{~d}^{-1}$, which supports the interpretation that this star is a hot and young star near the ZAMS and the blue edge of the classical instability strip (Zwintz et al. 2014). Yet without fundamental parameters from spectroscopy, we cannot infer if HD 46190 is a pre-main-sequence or mainsequence star. Once these data become available, and should a pre-main-sequence status be established, we can determine its properties based on the range of modes excited in the star. For pre-main-sequence stars, Zwintz et al. (2014) showed that there is a significant relationship between the pulsational properties and its evolution. Such a relation could not be established for $\delta$ Sct stars on the main-sequence or the post main-sequence.

\subsection{TIC $355687188=$ HD 224852}

The variability of the HADS star TIC 355687188 (HD 224852, BV 1007, NSV 14800, A8 V, V = 10.2) was discovered by Strohmeier (1967), but the nature of this variability was only revealed from the ASAS-3 observations by Pojmański (2002), who reported a period of $0.122072 \mathrm{~d}$ and classified the star as $\delta$ Sct. Poretti et al. (2005) carried out a detailed analysis of the ASAS-3 data and found the star to be 

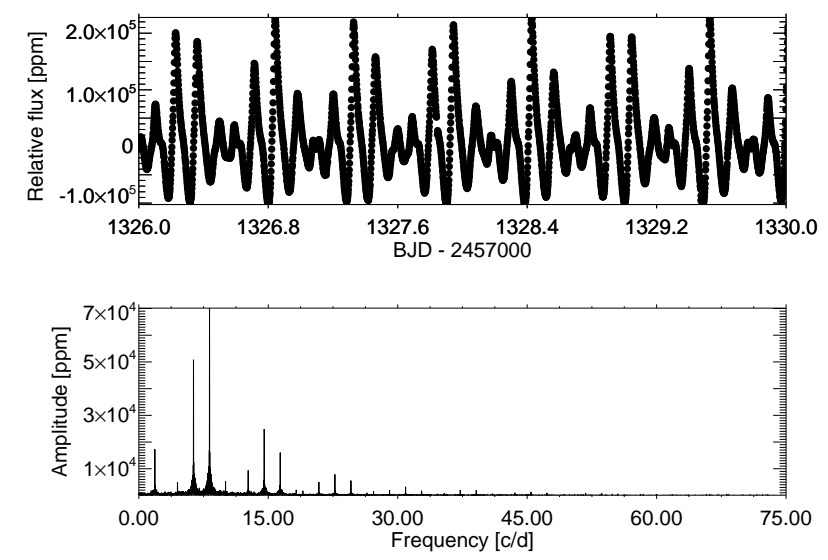

Figure 14. Upper panel: A 4-day section of the TESS light curve of HD 224852. The highly non-sinusoidal light curve and the beating between the two dominant modes are clearly visible. Lower panel: amplitude spectrum. The large majority of these peaks are combination frequencies and harmonics of the 4 independent modes. Although there are statistically significant peaks up to frequencies of $75 \mathrm{~d}^{-1}$, for illustrative reasons we only plot up to $75 \mathrm{~d}^{-1}$.

a double-mode pulsator with the frequency ratio of 0.77339 matching the ratio of the fundamental and first-overtone radial modes for HADS stars. In addition, the amplitude of the first overtone mode is higher than that of the fundamental mode. The full model describing the variability of the star in the ASAS data included 10 terms: the two radial mode frequencies, three harmonics of these frequencies and five combination frequencies.

HD 224852 was observed in Sectors 1 and 2 of the TESS mission with 2-min cadence; these data span $56.2 \mathrm{~d}$ with small ( 1-d) gaps in the middle and between each observing sector. A 4-d section of the TESS light curve is shown in Fig. 14, in which the strong beating of the two radial modes can be seen. Frequency analysis of the TESS data indicates that the observed variability in HD 224852 can be accounted for by only four independent modes, their harmonics and combination terms. In total, the model including all significant frequencies contains 96 sinusoidal terms yet only four independent pulsation modes (Table 6), 12 harmonics (of $f_{1}$ and $f_{2}$ only), and 80 combination frequencies.

Both radial modes have non-sinusoidal light curves, which is in accordance with the findings of Poretti et al. (2005). The first overtone mode has a larger amplitude than the fundamental mode, which is more commonly found for HADS stars in the LMC (Poleski et al. 2010) as opposed to the Milky Way. We infer that the independent frequencies $f_{3}$ and $f_{4}$ are non-radial modes. However, we cannot exclude that $f_{4}$ may be a higher-overtone radial mode. This can only be verified by detailed seismic modelling. We also note that no low-frequency modes were detected in HD 224852 above the detection threshold of $0.023 \mathrm{ppt}$ corresponding to $\mathrm{S} / \mathrm{N}$ $\approx 2.8$. The two radial modes show slight amplitude modulation during the TESS observations. In particular, the amplitude of $f_{1}$ drops by about 0.7 per cent and $f_{2}$ by about 0.4 per cent during this time. This effect has also been ob-
Table 6. Characterization of the amplitude spectrum of HD 224852 .

\begin{tabular}{ccccr}
\hline Mode & $\begin{array}{c}\text { Frequency } \\
{\left[\mathrm{d}^{-1}\right]}\end{array}$ & $\begin{array}{c}\text { Harmonics } \\
\text { detected }\end{array}$ & $\begin{array}{c}\text { Combinations } \\
\text { detected }\end{array}$ & $\mathrm{S} / \mathrm{N}$ \\
\hline$f_{1}$ & $8.1919470(07)$ & 7 & 78 & 8855.39 \\
$f_{2}$ & $6.3355745(10)$ & 5 & 79 & 6414.12 \\
$f_{3}$ & $7.9646(05)$ & 0 & 5 & 14.47 \\
$f_{4}$ & $13.1380(18)$ & 0 & 0 & 4.28 \\
\hline
\end{tabular}

served for other high-amplitude radial pulsators, such the HADS star KIC 5950759 (Bowman 2017).

\section{7 $\quad$ TIC 219234987 $=\gamma$ Dor}

$\gamma$ Dor is a bright $(V=4.20) \mathrm{F} 1 \mathrm{~V}$ (Gray et al. 2006) and is eponymous for an entire class of gravity/Rossby modes pulsators, the $\gamma$ Dor stars. Although this paper concentrates only on sectors 1 and 2, we decided to make an exception and to also include this prototype in this first-light article.

TESS observed $\gamma$ Dor in sectors 3, 4 and 5. The light curve was extracted by performing aperture photometry on target pixel files downloaded from MAST using the LIGHTCURVE python package (Barentsen et al. 2019). Bright as it is, $\gamma$ Dor saturates TESS CCDs. We settled on a classic approach for the preliminary analysis presented here. The photometric data extraction will be refined in the future using the halo photometry technique (White et al. 2017). The frequency analysis was subsequently done following an iterative pre-whitening procedure with the PERIOD04 software. The light curve and amplitude spectrum are plotted in Fig. 15 along with the 17 extracted frequencies with $\mathrm{S} / \mathrm{N}$ $\geqslant 4$.

$\gamma$ Dor has been extensively studied in the literature. Ground-based photometric (Cousins 1992; Balona et al. 1994a,b; Tarrant et al. 2008) and spectroscopic observations (Balona et al. 1996; Brunsden et al. 2018) dedicated to this star allowed the identification of a handful of frequencies. Brunsden et al. (2018) recently performed a comprehensive study of all available ground-based data. They found four consistent frequencies $\left(1.3209,1.3641,1.4742,1.8783 \mathrm{~d}^{-1}\right)$ that can all be confirmed with TESS data. The frequency at $0.31672 \mathrm{~d}^{-1}$, previously identified as a daily alias, is also present here, suggesting that it is indeed intrinsic to the star. We also corroborate the $2.7428 \mathrm{~d}^{-1}$ combination frequency. The amplitude spectrum shows amplitude excess at multiples of the main frequency group around $\sim 1.3 \mathrm{~d}^{-1}$, which is most likely due to harmonic and combination frequencies (e.g. Kurtz et al. 2015; Saio et al. 2018b), however, we cannot explore this further given the limited frequency resolution. Nevertheless, based on the observations and theory by $\mathrm{Li}$ et al. (2019b) and Saio et al. (2018b), we can speculate that the power excess around $1.4 \mathrm{~d}^{-1}$ and $2.8 \mathrm{~d}^{-1}$ could correspond to prograde $\mathrm{l}=1$ and $\mathrm{l}=2 \mathrm{~g}$ modes, respectively. Further observations are needed to resolve the modes and confirm this hypothesis. No significant p modes were detected, ruling out a hybrid nature. TESS observations in synergy with available spectroscopic (e.g. Gray et al. 2006; Ammler-von Eiff \& Reiners 2012) and astrometric (Gaia Collaboration et al. 2018) measurements will be presented in a forthcoming paper. 

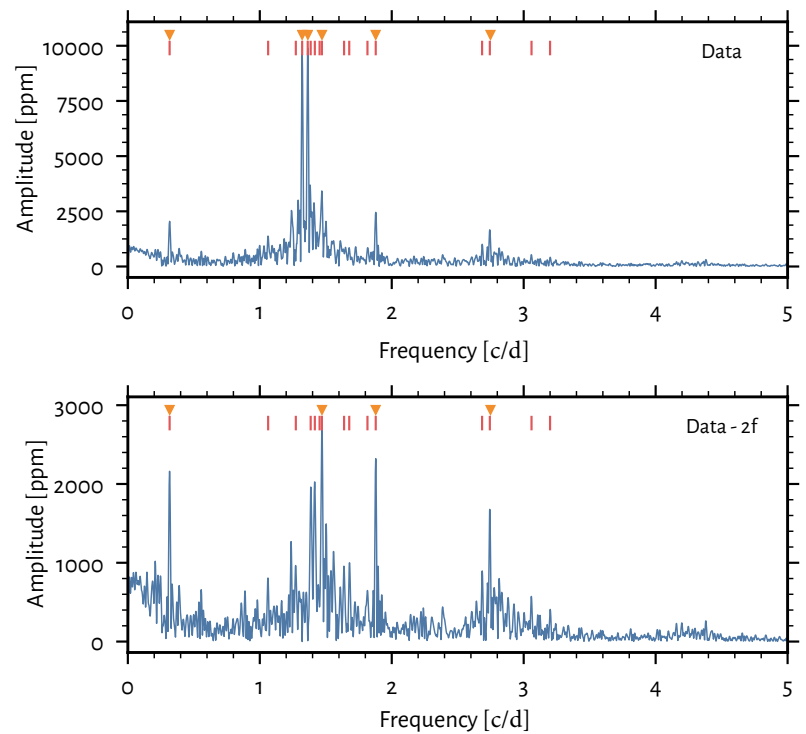

Figure 15. Amplitude Spectra of $\gamma$ Doradus from TESS observations before $(t o p)$ and after pre-whitening the first two frequencies (bottom). Red vertical bars mark the 17 frequencies with $\mathrm{S} / \mathrm{N}>4$ extracted from TESS data. Orange triangles indicate frequencies found in Brunsden et al. (2018).

\subsection{TIC $154842794=\pi$ PsA}

TIC 154842794 ( $\pi$ PsA, HD 217792) is a F1V type star (Gray et al. 2006) and a newly classified $\gamma$ Dor pulsator. (David \& Hillenbrand 2015a) find the following stellar parameters: $T_{\text {eff }}=7400 \pm 250 \mathrm{~K}, \log g=4.3$, and $M=1.5 \mathrm{M}_{\odot}$, with the effective temperature agreeing well with our determination of $T_{\text {eff }}=7440 \pm 110 \mathrm{~K}$. Additional values that may be relevant for the reader are $v_{\text {turb }}=2 \mathrm{~km} \mathrm{~s}^{-1}$ from Gray et al. (2006) and $[\mathrm{Fe} / \mathrm{H}]=-0.25 \pm 0.06$ from Gáspár et al. (2016) and a radius of $R=0.87 \mathrm{R}_{\odot}$ from Pasinetti Fracassini et al. (2001). We note that the radius of this star may be underestimated, as it is unrealistically low for an early $\mathrm{F}$ type star on or near the main-sequence.

Strohmeier et al. (1965) classified the star as a Cepheid with a period of $7.975 \mathrm{~d}$ and amplitude of $0.3 \mathrm{mag}$. It is thus likely the complex beating of the low-amplitude pulsations resulted in the misclassification. $\pi$ PsA is a known spectroscopic binary (Buscombe \& Morris 1961) with a low mass companion and an orbital period of $178 \mathrm{~d}$ (Allen et al. 2012; Pourbaix et al. 2004a) and eccentricity 0.53 (Abt 2005). The velocity amplitude of the primary is $21.3 \mathrm{~km} \mathrm{~s}^{-1}$ (Pourbaix et al. 2004a). It has also been flagged as a possible astrometric binary (Jancart et al. 2005). None of the frequencies are multiples of the orbital frequency. The star has an IR excess (Chen et al. 2014; Trilling et al. 2007), indicating the presence of a debris disk around the star and has been identified as a variable with a frequency of $0.94305 \mathrm{~d}^{-1}$ (Koen \& Eyer 2002). This frequency was confirmed using the original Hipparcos data. The sole Hipparcos frequency, calculated from 104 data-points, is inconsistent with any of the frequencies found in the TESS data.

The TESS light curve indicates the presence of a flare at $\mathrm{BJD}=2458370.162$ (Fig. 16), which might originate either

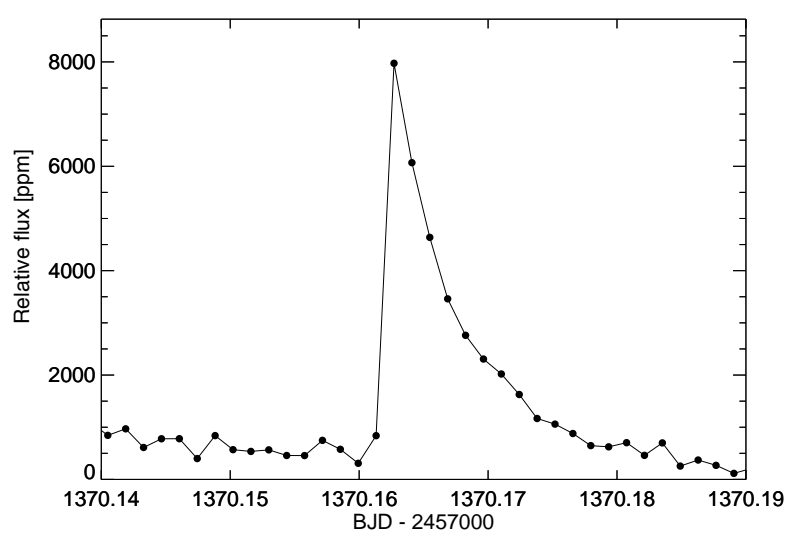

Figure 16. Flare observed in the TESS data of $\pi$ PsA.
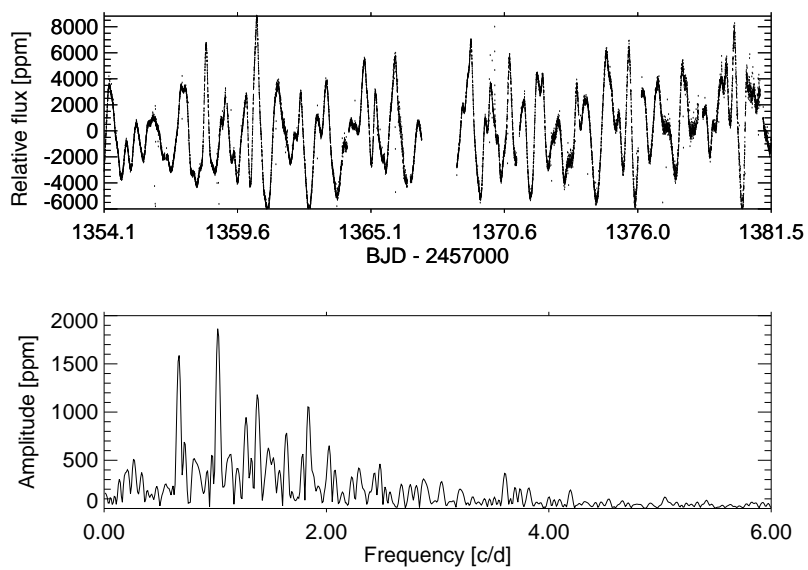

Figure 17. $\pi$ PsA. Upper panel: here we show the light curve that clearly resembles the variability of a typical $\gamma$ Dor star. Lower panel: Fourier spectrum of the TESS light curve. Here we find 6 statistically significant frequencies. See text for details.

from a background star or a possible companion. Detailed analyses as described in Pedersen et al. (2017) are required to identify the flare origin, but are out of the scope of this paper.

Based on TESS observations we find six statistically significant frequencies, which are $0.6721(2), 1.0242(2)$, $1.2688(3), 1.3814(3), 1.6385(5), 1.8376(3) \mathrm{d}^{-1}$, with additional peaks that are unfortunately unresolved, as this star was only observed for 27 days (Fig. 17). Based on the extracted peaks no clear period spacing can be detected. This shows that $\gamma$ Dor stars require longer observations.

\section{CONCLUSIONS}

We highlighted the different aspects of the pulsational variability of intermediate-mass A and F stars observed by the TESS mission in its first two sectors. The 2-min cadence of the TESS mission data is particularly useful for studying high-frequency pulsations in $\delta$ Sct stars; many modes are 
found which are often higher than the Nyquist frequency of $24.5 \mathrm{~d}^{-1}$ provided by 29.4-min cadence data of the Kepler mission (e.g., Bowman \& Kurtz 2018; Michel et al. 2017). The homogenous, all-sky sample of $\delta$ Sct stars observed by TESS is a significant advantage over previous ensembles of stars observed by, for example, the Kepler mission, and offers opportunities to test the physics of pulsation driving for a range in stellar mass, metallicity and rotation. In addition we used Gaia DR2 data and SED $T_{\text {eff }}$ values to calculate stellar luminosities and place our sample in the HR diagram (Fig. 2).

In this study, we have also demonstrated that using models with time-dependent non-local convection treatment and mimicking He depletion in the outer envelopes as expected for Am stars, we can explain the driving of pulsations in these stars. The distribution of 31 known Am stars observed by TESS in the HR diagram is consistent with our theoretical predictions, which show that turbulent pressure plays a very important role. Driving pulsations in Am stars has been a long-lasting mystery as the $\kappa$ mechanism alone could not explain the observations in the presence of $\mathrm{He}$ depletion through gravitational settling. Furthermore, the parameter space for which pulsation driving by turbulent pressure is predicted to be at its strongest is shown to be in the centre of the classical instability strip, corresponding to $T_{\text {eff }} \simeq 7500 \mathrm{~K}$. This is compatible with the parameter space for which the pulsator fraction also reaches its maximum of $\sim 70$ per cent within the classical instability strip (Murphy et al. 2019).

We highlighted the unprecedented asteroseismic potential that the TESS mission data provides for $\mathrm{A}$ and $\mathrm{F}$ stars, especially for pulsators that have previously not been observed with high-precision space telescopes such as the brightest known $\delta$ Sct and $\gamma$ Dor stars (e.g., $\alpha$ Pic, $\beta$ Pic, $\gamma$ Dor, etc). In addition, the near full-sky TESS observations will allow us to perform unbiased variability studies of A and F stars in general, but also in particular of, e.g., pre mainsequence and chemically peculiar stars, such as Am and Ap stars. We provided case studies of different variability types, including pulsators in eclipsing binaries, high-amplitude pulsators, chemically peculiar stars, and potential pre-mainsequence stars. We also show that the rather short observing periods of each sector (27 days) impose in some cases severe constraints on our ability to perform asteroseismic studies of, e.g., $\gamma$ Dor, $\delta$ Sct or hybrid stars due to their unresolved pulsation spectra. A limited frequency resolution will prevent us from detecting consecutive radial orders of gravity and Rossby modes as well as rotational splitting in pressure modes. Future studies using longer TESS light curves (obtained during the nominal and a possible extended mission), which are necessary for detailed asteroseismic modelling, will naturally build on this work and improve stellar structure and evolution theory for intermediate-mass stars.

\section{ACKNOWLEDGEMENTS}

We thank the referee for useful comments and discussions. This paper includes data collected by the TESS mission. Funding for the TESS mission is provided by the NASA Explorer Program. Funding for the TESS Asteroseismic Science Operations Centre is provided by the Danish National Re- search Foundation (Grant agreement no.: DNRF106), ESA PRODEX (PEA 4000119301) and Stellar Astrophysics Centre (SAC) at Aarhus University. We thank the TESS and TASC/TASOC teams for their support of the present work. This research has made use of the SIMBAD database, operated at CDS, Strasbourg, France. Some of the data presented in this paper were obtained from the Mikulski Archive for Space Telescopes (MAST). STScI is operated by the Association of Universities for Research in Astronomy, Inc., under NASA contract NAS5-2655.

Funding for the Stellar Astrophysics Centre is provided by The Danish National Research Foundation (Grant agreement no.: DNRF106). MC was supported by FCT - Fundação para a Ciência e a Tecnologia through national funds and by FEDER through COMPETE2020 Programa Operacional Competitividade e Internacionalização by these grants: UID/FIS/04434/2019, PTDC/FISAST/30389/2017 \& POCI-01-0145-FEDER-030389. MC is supported in the form of work contract funded by national funds through FCT (CEECIND/02619/2017). JDD acknowledges support from the Polish National Science Center (NCN), grant no. 2018/29/B/ST9/02803. AGH acknowledges funding support from Spanish public funds for research under projects ESP2017-87676-2-2 and ESP201565712-C5-5-R of the Spanish Ministry of Science and Education. FKA gratefully acknowledge funding through grant 2015/18/A/ST9/00578 of the Polish National Science Centre (NCN). JPe acknowledges funding support from the NSF REU program under grant number PHY-1359195. APi and KK acknowledge support provided by the Polish National Science Center (NCN) grant No. 2016/21/B/ST9/01126. This project has been supported by the Lendület Program of the Hungarian Academy of Sciences, project No. LP2018-7/2018, and by the support provided from the National Research, Development and Innovation Fund of Hungary, financed under the K16 funding scheme, project No. 115709. JCS acknowledges funding support from Spanish public funds for research under projects ESP2017-87676-2-2 and ESP2015- 65712-C5-5-R, and from project RYC-201209913 under the 'Ramón y Cajal' program of the Spanish Ministry of Science and Education. The research leading to these results has (partially) received funding from the European Research Council (ERC) under the European Union's Horizon 2020 research and innovation programme (grant agreement $\mathrm{N}^{\circ} 670519$ : MAMSIE), from the KU Leuven Research Council (grant C16/18/005: PARADISE), from the Research Foundation Flanders (FWO) under grant agreement G0H5416N (ERC Runner Up Project), as well as from the BELgian federal Science Policy Office (BELSPO) through PRODEX grant PLATO. acknowledges support by the Spanish State Research Agency (AEI) through project No. ESP2017-87676-C5-1-R and No MDM-20170737 Unidad de Excelencia María de Maeztut-Centro de Astrobiología (CSIC-INTA).ZsB acknowledges the support provided from the National Research, Development and Innovation Fund of Hungary, financed under the PD1717 funding scheme, project no. PD-123910. DLB acknowledges support from the Whitaker Foundation. SC gratefully acknowledge funding through grant 2015/18/A/ST9/00578 of the Polish National Science Centre (NCN). CCL gratefully acknowledges support from the Natural Sciences and Engineering Research Council of Canada. GMM acknowledges 
fundingby the STFC consolidated grant ST/R000603/1. RMO, SC and DR were supported in this work by the "Programme National de Physique Stellaire" (PNPS) of CNRS/INSU co-funded by CEA and CNES. IS acknowledges the partial support of projects DN 08-1/2016 and DN 18/13-12.12.2017. PS acknowledges financial support by the Polish NCN grant 2015/18/A/ST9/00578. MS acknowledges the Postdoc@MUNI project CZ.02.2.69/0.0/0.0/16027/0008360. JPG, JRR and MLM acknowledges funding support from Spanish public funds for research under project ESP2017-87676-C5-5-R and from the State Agency for Research of the Spanish MCIU through the "Center of Excellence Severo Ochoa" award for the Instituto de Astrofísica de Andalucía (SEV-2017-0709). JAE acknowledges STFC for funding support (reference ST/N504348/1). LFM acknowledges the financial support from the DGAPA-UNAM under grant PAPIIT IN100918. DM acknowledges his work as part of the research activity of the National Astronomical Research Institute of Thailand (NARIT), which is supported by the Ministry of Science and Technology of Thailand. MR acknowledges the support of the French Agence Nationale de la Recherche (ANR), under grant ESRR (ANR16-CE31-0007-01). We acknowledge the International Space Science Institute (ISSI) for supporting the SoFAR international team http://www.issi.unibe.ch/teams/sofar/. SBF acknowledges support by the Spanish State Research Agency (AEI) through project No. 'ESP2017-87676-C5-1R' and No MDM-2017-0737 Unidad de Excelencia 'María de Maeztu'-Centro de Astrobiología (CSIC-INTA).

This work has made use of data from the European Space Agency (ESA) mission Gaia (https://www.cosmos.esa.int/gaia), processed by the Gaia Data Processing and Analysis Consortium (DPAC, https://www.cosmos.esa.int/web/gaia/dpac/consortium).

Funding for the DPAC has been provided by national institutions, in particular the institutions participating in the Gaia Multilateral Agreement. The research leading to these results has received funding from the European Research Council (ERC) under the European Union's Horizon 2020 research and innovation programme (grant agreement No. 670519: MAMSIE) and from the Fonds Wetenschappelijk Onderzoek - Vlaanderen (FWO) under the grant agreement G0H5416N (ERC Opvangproject). This research has made use of the VizieR catalogue access tool, CDS, Strasbourg, France (DOI: 10.26093/cds/vizier). The original description of the VizieR service was published in A\&AS 143, 23.

This publication makes use of data products from the Two Micron All Sky Survey, which is a joint project of the University of Massachusetts and the Infrared Processing and Analysis Center/California Institute of Technology, funded by the National Aeronautics and Space Administration and the National Science Foundation.

\section{REFERENCES}

Abt H. A., 2000, ApJ, 544, 933

Abt H. A., 2005, ApJ, 629, 507

Aerts C., Christensen-Dalsgaard J., Kurtz D. W., 2010, Asteroseismology. Springer

Aerts C., Van Reeth T., Tkachenko A., 2017, ApJ, 847, L7

Aerts C., Mathis S., Rogers T., 2018a, ARA\&A, in press,
Aerts C., et al., 2018b, ApJS, 237, 15

Allen P. R., Burgasser A. J., Faherty J. K., Kirkpatrick J. D., 2012, AJ, 144, 62

Ammler-von Eiff M., Reiners A., 2012, A\&A, 542, A116

Ammons S. M., Robinson S. E., Strader J., Laughlin G., Fischer D., Wolf A., 2006, ApJ, 638, 1004

Andrievsky S. M., et al., 2002, A\&A, 396, 641

Antoci V., et al., 2011, Nature, 477, 570

Antoci V., et al., 2014, ApJ, 796, 118

Arenou F., Grenon M., Gomez A., 1992, A\&A, 258, 104

Arenou F., et al., 2018, A\&A, 616, A17

Asplund M., Grevesse N., Sauval A. J., Scott P., 2009, ARA\&A, 47,481

Aurière M., et al., 2010, A\&A, 523, A40

Auvergne M., et al., 2009, A\&A, 506, 411

Baglin A., Breger M., Chevalier C., Hauck B., Le Contel J. M., Sareyan J. P., Valtier J. C., 1973, A\&A, 23, 221

Bailer-Jones C. A., 2011, Monthly Notices of the Royal Astronomical Society, 411, 435

Balmforth N. J., Cunha M. S., Dolez N., Gough D. O., Vauclair S., 2001, MNRAS, 323, 362

Balona L. A., 2014, MNRAS, 439, 3453

Balona L. A., 2016, MNRAS, 459, 1097

Balona L. A., Dziembowski W. A., 2011, MNRAS, 417, 591

Balona L. A., Hearnshaw J. B., Koen C., Collier A., Machi I., Mkhosi M., Steenberg C., 1994a, MNRAS, 267, 103

Balona L. A., Krisciunas K., Cousins A. W. J., 1994b, MNRAS, 270,905

Balona L. A., et al., 1996, MNRAS, 281, 1315

Balona L. A., et al., 2012, MNRAS, 419, 3028

Balona L. A., Daszyńska-Daszkiewicz J., Pamyatnykh A. A., 2015, MNRAS, 452, 3073

Barceló Forteza S., Michel E., Roca Cortés T., García R. A., 2015, A\&A, 579, A133

Barceló Forteza S., Roca Cortés T., García Hernández A., García R. A., 2017, A\&A, 601, A57

Barceló Forteza S., Roca Cortés T., García R. A., 2018, A\&A, 614, A46

Barentsen G., et al., 2019, KeplerGO/lightkurve: Lightkurve v1.0b29, doi:10.5281/zenodo.2565212, https://doi.org/10. 5281 /zenodo. 2565212

Bigot L., Dziembowski W. A., 2002, A\&A, 391, 235

Bohlender D. A., Gonzalez J.-F., Matthews J. M., 1999, A\&A, 350,553

Bowman D. M., 2017, Amplitude Modulation of Pulsation Modes in Delta Scuti Stars. Springer International Publishing, 2017, doi:10.1007/978-3-319-66649-5

Bowman D. M., Kurtz D. W., 2018, MNRAS, 476, 3169

Bowman D. M., Holdsworth D. L., Kurtz D. W., 2015, MNRAS, 449, 1004

Bowman D. M., Kurtz D. W., Breger M., Murphy S. J., Holdsworth D. L., 2016, MNRAS, 460, 1970

Bowman D. M., Buysschaert B., Neiner C., Pápics P. I., Oksala M. E., Aerts C., 2018, A\&A, 616, A77

Bowman D. M., et al., 2019, A\&A, 621, A135

Breger M., 1970, ApJ, 162, 597

Breger M., 2000, in Breger M., Montgomery M., eds, Astronomical Society of the Pacific Conference Series Vol. 210, Delta Scuti and Related Stars. p. 3

Breger M., et al., 1993, A\&A, 271, 482

Breger M., Beck P., Lenz P., Schmitzberger L., Guggenberger E., Shobbrook R. R., 2006, A\&A, 455, 673

Breger M., et al., 2011, MNRAS, 414, 1721

Brunsden E., Pollard K. R., Wright D. J., De Cat P., Cottrell P. L., 2018, MNRAS, 475, 3813

Buscombe W., Morris P. M., 1961, MNRAS, 123, 183

Buzasi D. L., et al., 2005, ApJ, 619, 1072 
Casagrande L., Schönrich R., Asplund M., Cassisi S., Ramírez I., Meléndez J., Bensby T., Feltzing S., 2011, A\&A, 530, A138 Chaplin W. J., Miglio A., 2013, ARA\&A, 51, 353

Chen B., Vergely J. L., Valette B., Carraro G., 1998, A\&A, 336, 137

Chen C. H., Mittal T., Kuchner M., Forrest W. J., Lisse C. M., Manoj P., Sargent B. A., Watson D. M., 2014, ApJS, 211, 25

Cheng K.-P., Neff J. E., Johnson D. M., Tarbell E. S., Romo C. A., Gray R. O., Corbally C. J., 2017, AJ, 153, 39

Christophe S., Ballot J., Ouazzani R.-M., Antoci V., Salmon S. J. A. J., 2018, A\&A, 618, A47

Cohen R. E., Sarajedini A., 2012, MNRAS, 419, 342

Conti P. S., 1970, PASP, 82, 781

Corbally C., 1984, The Astronomical Journal, 89, 1887

Cousins A. W. J., 1992, The Observatory, 112, 53

Cox J. P., 1963, ApJ, 138, 487

Cunha M. S., 2001, MNRAS, 325, 373

Cunha M. S., 2006, MNRAS, 365, 153

Cunha M. S., Gough D., 2000, MNRAS, 319, 1020

Cunha M. S., et al., 2019, MNRAS, 487, 3523

David T. J., Hillenbrand L. A., 2015a, ApJ, 804, 146

David T. J., Hillenbrand L. A., 2015b, ApJ, 804, 146

Deeming T. J., 1975, Ap\&SS, 36, 137

Degroote P., et al., 2009, A\&A, 506, 111

Díaz C. G., González J. F., Levato H., Grosso M., 2011, A\&A, 531, A143

Drimmel R., Cabrera-Lavers A., López-Corredoira M., 2003, A\&A, 409, 205

Dupret M.-A., Grigahcène A., Garrido R., Gabriel M., Scuflaire R., 2005, A\&A, 435, 927

Dziembowski W., 1977, Acta Astron., 27, 95

Eggleton P. P., Tokovinin A. A., 2008, MNRAS, 389, 869

Erspamer D., North P., 2003, Astronomy \& Astrophysics, 398, 1121

Evans D. W., Irwin M. J., Helmer L., 2002, A\&A, 395, 347

Faraggiana R., Bonifacio P., Caffau E., Gerbaldi M., Nonino M., 2004, Astronomy \& Astrophysics, 425, 615

Flower P. J., 1996, ApJ, 469, 355

Frandsen S., Jones A., Kjeldsen H., Viskum M., Hjorth J., Andersen N. H., Thomsen B., 1995, A\&A, 301, 123

Gaia Collaboration 2018, VizieR Online Data Catalog, 1345

Gaia Collaboration et al., 2018, A\&A, 616, A1

García Hernández A., Martín-Ruiz S., Monteiro M. J. P. F. G., Suárez J. C., Reese D. R., Pascual-Granado J., Garrido R., 2015, ApJ, 811, L29

García Hernández A., et al., 2017, MNRAS, 471, L140

Gáspár A., Rieke G. H., Ballering N., 2016, ApJ, 826, 171

González J. F., Hubrig S., Savanov I., 2008, Contributions of the Astronomical Observatory Skalnate Pleso, 38, 411

Gough D. O., 1977a, in Spiegel E. A., Zahn J.-P., eds, Lecture Notes in Physics, Berlin Springer Verlag Vol. 71, Problems of Stellar Convection. pp 349-363, doi:10.1007/3-540-08532-7_56

Gough D. O., 1977b, ApJ, 214, 196

Gray D. F., 2008, The Observation and Analysis of Stellar Photospheres. Cambridge University Press

Gray R. O., Corbally C. J., 1998, AJ, 116, 2530

Gray R. O., Corbally C. J., Garrison R. F., McFadden M. T., Bubar E. J., McGahee C. E., O'Donoghue A. A., Knox E. R., 2006, AJ, 132, 161

Green G. M., et al., 2015, ApJ, 810, 25

Green G. M., et al., 2018, MNRAS, 478, 651

Grigahcène A., et al., 2010, ApJ, 713, L192

Gruber D., et al., 2012, MNRAS, 420, 291

Guenther D. B., Kallinger T., Zwintz K., Weiss W. W., Tanner J., 2007, ApJ, 671, 581

Guo Z., Gies D. R., Matson R. A., García Hernández A., 2016, ApJ, 826, 69
Guo Z., Gies D. R., Matson R. A., García Hernández A., Han Z., Chen X., 2017, ApJ, 837, 114

Guzik J. A., Kaye A. B., Bradley P. A., Cox A. N., Neuforge C., 2000, ApJ, 542, L57

Handberg R., 2013, PhD thesis, Aarhus University

Hatta Y., Sekii T., Takata M., Kurtz D. W., 2019, ApJ, 871, 135

Heiter U., 2002, A\&A, 381, 959

Heiter U., et al., 2002, A\&A, 392, 619

Helt B. E., 1984, A\&AS, 56, 457

Henden A. A., Levine S., Terrell D., Welch D. L., 2015, in American Astronomical Society Meeting Abstracts \#225. p. 336.16

Henden A. A., Templeton M., Terrell D., Smith T. C., Levine S., Welch D., 2016, VizieR Online Data Catalog, 2336

Hoeg E., et al., 1997, A\&A, 323, L57

Houdek G., 2000, in Breger M., Montgomery M., eds, Astronomical Society of the Pacific Conference Series Vol. 210, Delta Scuti and Related Stars. p. 454

Houdek G., Balmforth N. J., Christensen-Dalsgaard J., Gough D. O., 1999, A\&A, 351, 582

Howarth I. D., 1983, MNRAS, 203, 301

Howell S. B., et al., 2014, PASP, 126, 398

Huber D., et al., 2016, The Astrophysical Journal Supplement Series, 224, 2

Jancart S., Jorissen A., Babusiaux C., Pourbaix D., 2005, A\&A, 442,365

Kallinger T., et al., 2017, A\&A, 603, A13

Kama M., Folsom C. P., Pinilla P., 2015, A\&A, 582, L10

Kamath D., Wood P. R., Van Winckel H., 2014, MNRAS, 439, 2211

Kharchenko N. V., 2001, Kinematika i Fizika Nebesnykh Tel, 17, 409

Kim C., McNamara D. H., Christensen C. G., 1993, AJ, 106, 2493

King J. R., 1994, MNRAS, 269, 209

Koch D. G., et al., 2010, ApJ, 713, L79

Koen C., Eyer L., 2002, MNRAS, 331, 45

Kopacki G., 2015, Acta Astron., 65, 81

Kordopatis G., et al., 2013, AJ, 146, 134

Kunder A., et al., 2017, VizieR Online Data Catalog, 3279

Kurtz D. W., 1978, ApJ, 221, 869

Kurtz D. W., 1982, MNRAS, 200, 807

Kurtz D. W., 1985, MNRAS, 213, 773

Kurtz D. W., Saio H., Takata M., Shibahashi H., Murphy S. J., Sekii T., 2014, MNRAS, 444, 102

Kurtz D. W., Shibahashi H., Murphy S. J., Bedding T. R., Bowman D. M., 2015, MNRAS, 450, 3015

Kurucz R., 1993, ATLAS9 Stellar Atmosphere Programs and 2 $\mathrm{km} / \mathrm{s}$ grid. Kurucz CD-ROM No. 13. Cambridge, Mass.: Smithsonian Astrophysical Observatory, 1993., 13

Kurucz R. L., Avrett E. H., 1981, SAO Special Report, 391

Lee J. W., Hong K., Kristiansen M. H., 2019, AJ, 157, 17

Lenz P., Breger M., 2004, in Zverko J., Ziznovsky J., Adelman S. J., Weiss W. W., eds, IAU Symposium Vol. 224, The AStar Puzzle. pp 786-790, doi:10.1017/S1743921305009750

Lenz P., Pamyatnykh A. A., Breger M., Antoci V., 2008, A\&A, 478,855

Li G., Bedding T. R., Murphy S. J., Van Reeth T., Antoci V., Ouazzani R.-M., 2019a, MNRAS, 482, 1757

Li G., Van Reeth T., Bedding T. R., Murphy S. J., Antoci V., 2019b, MNRAS, 487, 782

Liakos A., Niarchos P., 2016, Monthly Notices of the Royal Astronomical Society, p. stw2756

Liakos A., Niarchos P., 2017, MNRAS, 465, 1181

Lindegren L., et al., 2016, A\&A, 595, A4

Lindegren L., et al., 2018, A\&A, 616, A2

Maeder A., 2009, Physics, Formation and Evolution of Rotating Stars. Springer, doi:10.1007/978-3-540-76949-1

Malkov O. Y., Tamazian V. S., Docobo J. A., Chulkov D. A., 2012, A\&A, 546, A69 
Marconi M., Palla F., 1998, ApJ, 507, L141

Martell S. L., et al., 2017, MNRAS, 465, 3203

Masana E., Jordi C., Ribas I., 2006, A\&A, 450, 735

Matthews J. M., 2007, Communications in Asteroseismology, 150, 333

McDonald I., Zijlstra A. A., Boyer M. L., 2012, MNRAS, 427, 343

McDonald I., Zijlstra A. A., Watson R. A., 2017, MNRAS, 471, 770

McNamara D. H., 2000, in Breger M., Montgomery M., eds, Astronomical Society of the Pacific Conference Series Vol. 210, Delta Scuti and Related Stars. p. 373

McNamara D. H., 2011, AJ, 142, 110

McNamara D. H., Clementini G., Marconi M., 2007, AJ, 133, 2752

Meynet G., Ekstrom S., Maeder A., Eggenberger P., Saio H., Chomienne V., Haemmerlé L., 2013, in Goupil M., Belkacem K., Neiner C., Lignières F., Green J. J., eds, Lecture Notes in Physics, Berlin Springer Verlag Vol. 865, Lecture Notes in Physics, Berlin Springer Verlag. p. 3 (arXiv:1301.2487), doi:10.1007/978-3-642-33380-4_1

Michaud G., 1970, ApJ, 160, 641

Michel E., et al., 2017, in European Physical Journal Web of Conferences. p. 03001 (arXiv:1705.03721), doi:10.1051/epjconf/201716003001

Mombarg J. S. G., Van Reeth T., Pedersen M. G., Molenberghs G., Bowman D. M., Johnston C., Tkachenko A., Aerts C., 2019, MNRAS,

Monet D. G., et al., 2003, AJ, 125, 984

Montgomery M. H., O'Donoghue D., 1999, Delta Scuti Star Newsletter, 13, 28

Munari U., et al., 2014, AJ, 148, 81

Murphy S. J., 2014, PhD thesis, Jeremiah Horrocks Institute, University of Central Lancashire, Preston, UK $<$ EMAIL > murphy@physics.usyd.edu.au </EMAIL >

Murphy S. J., 2015, MNRAS, 453, 2569

Murphy S. J., et al., 2013, MNRAS, 432, 2284

Murphy S. J., et al., 2015, Publ. Astron. Soc. Australia, 32, e036

Murphy S. J., Hey D., Van Reeth T., Bedding T. R., 2019, MNRAS,

Nemec J., Mateo M., 1990, in Cacciari C., Clementini G., eds, Astronomical Society of the Pacific Conference Series Vol. 11, Confrontation Between Stellar Pulsation and Evolution. pp 64-84

Nemec J. M., Nemec A. F. L., Lutz T. E., 1994, AJ, 108, 222

Nemec J. M., Balona L. A., Murphy S. J., Kinemuchi K., Jeon Y.-B., 2017, MNRAS, 466, 1290

Niemczura E., Scholz R. D., Hubrig S., Järvinen S. P., Schöller M., Ilyin I., Kahraman Aliçavuș F., 2017, MNRAS, 470, 3806

Oelkers R. J., et al., 2018, AJ, 155, 39

Ouazzani R. M., Dupret M. A., Reese D. R., 2012, A\&A, 547, A75

Ouazzani R.-M., Salmon S. J. A. J., Antoci V., Bedding T. R., Murphy S. J., Roxburgh I. W., 2017, MNRAS, 465, 2294

Pamyatnykh A. A., 1999, Acta Astron., 49, 119

Pamyatnykh A. A., 2000, in Breger M., Montgomery M., eds, Astronomical Society of the Pacific Conference Series Vol. 210, Delta Scuti and Related Stars. p. 215 (arXiv:astro$\mathrm{ph} / 0005276)$

Pamyatnykh A. A., Dziembowski W. A., Handler G., Pikall H., 1998, A\&A, 333, 141

Paparo M., Sterken C., Spoon H. W. W., Birch P. V., 1996, A\&A, 315,400

Paparó M., Benkő J. M., Hareter M., Guzik J. A., 2016a, ApJS, 224, 41

Paparó M., Benkő J. M., Hareter M., Guzik J. A., 2016b, ApJ, 822,100

Pápics P. I., et al., 2012, A\&A, 542, A55

Pasinetti Fracassini L. E., Pastori L., Covino S., Pozzi A., 2001,
A\&A, 367, 521

Paunzen E., Handler G., Weiss W. W., North P., 1994, Information Bulletin on Variable Stars, 4094

Paunzen E., et al., 2002, A\&A, 392, 515

Paunzen E., Heiter U., Fraga L., Pintado O., 2012, MNRAS, 419, 3604

Pedersen M. G., Antoci V., Korhonen H., White T. R., JessenHansen J., Lehtinen J., Nikbakhsh S., Viuho J., 2017, MNRAS, 466, 3060

Petersen J. O., Christensen-Dalsgaard J., 1996, A\&A, 312, 463

Pigulski A., 2014, in Guzik J. A., Chaplin W. J., Handler G., Pigulski A., eds, IAU Symposium Vol. 301, Precision Asteroseismology. pp 31-38 (arXiv:1311.3954), doi:10.1017/S1743921313014038

Pigulski A., Michalska G., 2007, Acta Astron., 57, 61

Pojmański G., 1997, Acta Astron., 47, 467

Pojmański G., 2002, Acta Astron., 52, 397

Poleski R., et al., 2010, Acta Astron., 60, 1

Poretti E., et al., 2005, A\&A, 440, 1097

Poretti E., et al., 2011, Astron. Astrophys., 528, A147

Pourbaix D., et al., 2004a, A\&A, 424, 727

Pourbaix D., et al., 2004b, Astronomy \& Astrophysics, 424, 727

Press W. H., Rybicki G. B., 1989, ApJ, 338, 277

Preston G. W., 1974, ARA\&A, 12, 257

Rainer M., et al., 2016, AJ, 152, 207

Rainer M., et al., 2017, VizieR Online Data Catalog, 515

Reegen P., 2007, A\&A, 467, 1353

Renson P., Manfroid J., 2009, Astron. Astrophys., 498, 961

Renson P., Gerbaldi M., Catalano F., 1991, Astronomy and Astrophysics Supplement Series, 89, 429

Ricker G. R., et al., 2014, in Space Telescopes and Instrumentation 2014: Optical, Infrared, and Millimeter Wave. p. 914320 (arXiv: 1406.0151), doi:10.1117/12.2063489

Ricker G. R., et al., 2015, Journal of Astronomical Telescopes, Instruments, and Systems, 1, 014003

Rodríguez E., López-González M., 2000, Astronomy and Astrophysics Supplement Series, 144, 469

Royer F., Grenier S., Baylac M.-O., Gómez A. E., Zorec J., 2002, A\&A, 393, 897

Royer F., Zorec J., Gómez A., 2007, Astronomy \& Astrophysics, 463,671

Saio H., 2005, MNRAS, 360, 1022

Saio H., Gautschy A., 2004, MNRAS, 350, 485

Saio H., Kurtz D. W., Takata M., Shibahashi H., Murphy S. J., Sekii T., Bedding T. R., 2015, MNRAS, 447, 3264

Saio H., Kurtz D. W., Murphy S. J., Antoci V. L., Lee U., 2018a, MNRAS, 474, 2774

Saio H., Bedding T. R., Kurtz D. W., Murphy S. J., Antoci V., Shibahashi H., Li G., Takata M., 2018b, MNRAS, 477, 2183 Scargle J. D., 1982, ApJ, 263, 835

Schlegel D. J., Finkbeiner D. P., Davis M., 1998, ApJ, 500, 525

Schmid V. S., Aerts C., 2016, A\&A, 592, A116

Schwarzenberg-Czerny A., 2003, in Sterken C., ed., Astronomical Society of the Pacific Conference Series Vol. 292, Interplay of Periodic, Cyclic and Stochastic Variability in Selected Areas of the H-R Diagram. p. 383

Shulyak D., Tsymbal V., Ryabchikova T., Stütz C., Weiss W. W., 2004, A\&A, 428, 993

Siebert A., et al., 2011, AJ, 141, 187

Sikora J., et al., 2019, arXiv e-prints, p. arXiv:1905.08835

Skrutskie M. F., et al., 2006, AJ, 131, 1163

Sloan G. C., et al., 2004, ApJ, 614, L77

Smalley B., et al., 2011, A\&A, 535, A3

Smalley B., et al., 2017, MNRAS, 465, 2662

Soubiran C., Le Campion J.-F., Cayrel de Strobel G., Caillo A., 2010, A\&A, 515, A111

Soubiran C., Le Campion J.-F., Brouillet N., Chemin L., 2016 , Astronomy \& Astrophysics, 591, A118 
Stassun K. G., et al., 2018, AJ, 156, 102

Stępień K., 2000, A\&A, 353, 227

Stevens D. J., Stassun K. G., Gaudi B. S., 2017, AJ, 154, 259

Strohmeier W., 1967, Information Bulletin on Variable Stars, 195

Strohmeier W., Knigge R., Ott H., 1965, Information Bulletin on Variable Stars, 86

Suárez J. C., García Hernández A., Moya A., Rodrigo C., Solano E., Garrido R., Rodón J. R., 2014, A\&A, 563, A7

Suran M., Goupil M., Baglin A., Lebreton Y., Catala C., 2001, A\&A, 372, 233

Tarrant N. J., Chaplin W. J., Elsworth Y. P., Spreckley S. A., Stevens I. R., 2008, A\&A, 492, 167

Templeton M., Basu S., Demarque P., 2002, ApJ, 576, 963

Tkachenko A., 2015, A\&A, 581, A129

Tokovinin A., Kiyaeva O., 2016, MNRAS, 456, 2070

Trilling D. E., et al., 2007, ApJ, 658, 1289

Tsymbal V., 1996, in Adelman S. J., Kupka F., Weiss W. W., eds, Astronomical Society of the Pacific Conference Series Vol. 108, M.A.S.S., Model Atmospheres and Spectrum Synthesis. p. 198

Turcotte S., Richer J., Michaud G., Christensen-Dalsgaard J., 2000, A\&A, 360, 603

Uesugi A., Fukuda I., 1970, Contributions from the Institute of Astrophysics and Kwasan Observatory, University of Kyoto, Kyoto: University, Kwasan Observatory, Institute of Astrophysics, 1970

Uytterhoeven K., et al., 2011, A\&A, 534, A125

Van Reeth T., et al., 2015a, ApJS, 218, 27

Van Reeth T., et al., 2015b, A\&A, 574, A17

Van Reeth T., Tkachenko A., Aerts C., 2016, A\&A, 593, A120

Van Reeth T., et al., 2018, A\&A, 618, A24

Venn K. A., Lambert D. L., 1990, ApJ, 363, 234

Weiss W. W., et al., 2014, PASP, 126, 573

White T. R., et al., 2017, MNRAS, 471, 2882

Xiong D. R., Deng L., Zhang C., Wang K., 2016, MNRAS, 457, 3163

Ziaali E., Bedding T. R., Murphy S. J., Van Reeth T., Hey D. R., 2019, MNRAS, 486, 4348

Zinn J. C., Pinsonneault M. H., Huber D., Stello D., 2019, ApJ, 878,136

Zorec J., Royer F., 2012a, A\&A, 537, A120

Zorec J., Royer F., 2012b, Astronomy \& Astrophysics, 537, A120

Zwintz K., Fossati L., Ryabchikova T., Kaiser A., Gruberbauer M., Barnes T. G., Baglin A., Chaintreuil S., 2013, A\&A, 550, A121

Zwintz K., et al., 2014, Science, 345, 550

Zwitter T., et al., 2010, A\&A, 522, A54

van Leeuwen F., 2007, A\&A, 474, 653

\section{APPENDIX A: TABLES}


Table A1: Stellar parameters for the TESS $\delta$ Sct and $\gamma$ Dor pulsators observed at 2-min cadence during the first two pointings

(Sectors 1 and 2) in the ecliptic Southern hemisphere. The columns indicate: the TESS Input Catalogue (TIC) number, the

and $\log g$ values and their associated uncertainties from literature respectively. The reference is indicated next to each value.

In addition, we list the projected rotational velocity and binarity status as available in literature.

\begin{tabular}{|c|c|c|c|c|c|c|c|c|c|c|c|c|c|}
\hline TIC & $\begin{array}{c}\text { TESS } \\
\text { mag }\end{array}$ & $\begin{array}{l}\text { alternative } \\
\text { identifier }\end{array}$ & $\begin{array}{l}T_{\text {eff }}[K] \\
\text { Spec. }\end{array}$ & $\begin{array}{l}\sigma_{T_{\text {eff }}} \\
\text { Spec. }\end{array}$ & $\begin{array}{l}T_{\text {eff }}[K] \\
\text { Phot. }\end{array}$ & $\begin{array}{l}\sigma_{T_{\text {eff }}} \\
\text { Phot. }\end{array}$ & $\begin{array}{l}\log g \\
\text { Spec. }\end{array}$ & $\begin{array}{l}\sigma_{\log g} \\
\text { Spec. }\end{array}$ & $\begin{array}{l}\log g \\
\text { Phot. }\end{array}$ & $\begin{array}{l}\sigma_{\log g} \\
\text { Phot. }\end{array}$ & $\begin{array}{c}v \sin i \\
{\left[\mathrm{~km} \mathrm{~s}^{-1}\right]}\end{array}$ & $\begin{array}{c}\sigma_{\mathrm{vsini}} \\
{\left[\mathrm{km} \mathrm{s}^{-1}\right]}\end{array}$ & binarity \\
\hline 9632550 & 9.06 & BS Aqr & $7245^{1}$ & $75^{1}$ & - & - & $3.82^{1}$ & $0.12^{1}$ & 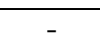 & - & $23^{1}$ & - & - \\
\hline 12470841 & 8.44 & HD 923 & - & - & $7832^{2}$ & - & - & - & - & - & - & - & - \\
\hline 12784216 & 8.92 & HD 213125 & - & - & $6519^{3}$ & $178^{3}$ & - & - & $4.03^{3}$ & $0.26^{3}$ & - & - & - \\
\hline 12974182 & 6.78 & HD 218003 & $7471^{4}$ & $95^{4}$ & $7584^{5}$ & $208^{5}$ & $3.86^{4}$ & $0.18^{4}$ & $4.05^{5}$ & - & - & - & - \\
\hline 29281339 & 6.63 & HD 198501 & - & - & $7511^{5}$ & $284^{5}$ & - & - & $3.88^{5}$ & - & - & - & - \\
\hline 30531417 & 11.03 & XX Dor & - & - & $6769^{6}$ & $202^{6}$ & - & - & - & $1.82^{6}$ & - & - & - \\
\hline 32176627 & 6.90 & HD 209970 & - & - & $7156^{5}$ & $347^{5}$ & - & - & $4.10^{5}$ & - & - & - & - \\
\hline 33911462 & 8.06 & HD 222828 & - & - & $6987^{7}$ & $98^{7}$ & - & - & $3.53^{7}$ & - & - & - & - \\
\hline 33984043 & 6.37 & HD 223466 & - & - & $8433^{8}$ & $58^{8}$ & - & - & $4.23^{9}$ & - & $69.5^{10}$ & $1^{10}$ & - \\
\hline 34038105 & 6.74 & HD 223991A & - & - & $8854^{11}$ & & - & - & - & - & - & - & \\
\hline 38587180 & 7.69 & TX Ret & $8009^{12}$ & - & $7258^{2}$ & - & $3.62^{12}$ & - & - & - & - & - & - \\
\hline 38602305 & 6.13 & $\theta$ Ret $\mathrm{A}$ & - & - & $12442^{2}$ & - & - & - & - & - & - & - & binary $^{13}$ \\
\hline 38847248 & 5.86 & $\theta$ Tuc & - & - & $7638^{14}$ & $90^{14}$ & - & - & $3.59^{14}$ & $0.08^{14}$ & $80^{15}$ & - & binary $^{16}$ \\
\hline 44627561 & 8.98 & HD 215559 & - & - & - & - & - & - & - & - & - & - & - \\
\hline 49677785 & 9.35 & HD 220687 & $7033^{1}$ & $77^{1}$ & - & - & $3.39^{1}$ & $0.12^{1}$ & - & - & $91^{1}$ & - & - \\
\hline 51991595 & 12.07 & 2MASS J01011055-6843069 & - & - & $6794^{6}$ & $201^{6}$ & - & - & $4.19^{6}$ & - & - & - & - \\
\hline 52258534 & 7.71 & BG Hyi & $7289^{12}$ & - & $6928^{2}$ & - & $2.91^{12}$ & - & - & - & - & - & - \\
\hline 66434034 & 8.88 & HD 5497 & - & - & - & - & - & - & - & - & - & - & - \\
\hline 70744515 & 8.80 & HD 225077 & - & - & - & - & - & - & - & - & - & - & - \\
\hline 71334169 & 8.52 & HD 209430 & - & - & $6872^{17}$ & $125^{17}$ & - & - & - & - & - & - & - \\
\hline 79394646 & 3.58 & $\theta$ Ind $\mathrm{A}$ & $7870^{18}$ & - & - & - & $4.14^{18}$ & - & - & - & $135^{10}$ & $2^{10}$ & binary $^{19}$ \\
\hline 80474886 & 6.23 & AZ Phe & - & - & $7278^{14}$ & $35^{14}$ & - & - & $3.96^{5}$ & $0.11^{14}$ & & - & - \\
\hline 88277481 & 9.04 & HD 210740 & - & - & - & - & - & - & - & - & - & - & - \\
\hline 89464315 & 5.29 & WX PsA & $7430^{39}$ & $34^{39}$ & $7509^{9}$ & $255^{9}$ & - & - & $3.67^{9}$ & $0.14^{9}$ & $91^{8}$ & - & - \\
\hline 89542582 & 9.71 & HD 223587 & - & - & $7295^{5}$ & $208^{5}$ & - & - & $3.97^{5}$ & - & - & - & - \\
\hline 92734713 & 8.32 & HD 200835 & - & - & - & - & - & - & - & - & - & - & - \\
\hline 99839685 & 8.46 & HD 204972 & - & - & $7153^{2}$ & - & - & - & - & - & - & - & - \\
\hline 102090493 & 9.20 & HD 7454 & - & - & $7195^{11}$ & - & - & - & - & - & - & - & - \\
\hline 116157537 & 5.88 & BB Phe & $6973^{39}$ & $107^{39}$ & $7000^{2}$ & - & - & - & - & - & $83^{15}$ & - & - \\
\hline 126659093 & 9.18 & ZZ Mic & $7217^{1}$ & $54^{1}$ & $7613^{11}$ & - & - & - & - & - & $35^{1}$ & - & - \\
\hline 137796620 & 9.87 & HD 216728 & - & - & $7559^{6}$ & $213^{6}$ & - & - & $4.13^{6}$ & $0.48^{6}$ & - & - & - \\
\hline 139131968 & 5.52 & $\mathrm{Tau}^{3}$ Gru & - & - & $7820^{21}$ & $100^{21}$ & - & - & $3.723^{5}$ & - & - & - & - \\
\hline 139232635 & 3.94 & $\theta$ Gru & - & - & - & - & - & - & - & - & $64^{22}$ & - & binary ${ }^{19}$ \\
\hline 139825582 & 7.40 & CF Ind & $6938^{23}$ & - & $7075^{2}$ & - & $3.3^{23}$ & - & - & - & - & - & - \\
\hline
\end{tabular}


Table A1: Stellar parameters for the TESS $\delta$ Sct and $\gamma$ Dor pulsators observed at 2-min cadence during the first two pointings (Sectors 1 and 2) in the ecliptic Southern hemisphere. The columns indicate: the TESS Input Catalogue (TIC) number, the TESS magnitude, an alternative identifier of the star, spectroscopic (Spec.) and photometric (Phot) effective temperatures and $\log g$ values and their associated uncertainties from literature respectively. The reference is indicated next to each value. In addition, we list the projected rotational velocity and binarity status as available in literature.

\begin{tabular}{|c|c|c|c|c|c|c|c|c|c|c|c|c|c|}
\hline TIC & $\begin{array}{c}\text { TESS } \\
\text { mag }\end{array}$ & $\begin{array}{l}\text { alternative } \\
\text { identifier }\end{array}$ & $\begin{array}{l}T_{\text {eff }}[K] \\
\text { Spec. }\end{array}$ & $\begin{array}{l}\sigma_{T_{\text {eff }}} \\
\text { Spec. }\end{array}$ & $\begin{array}{l}T_{\text {eff }}[K] \\
\text { Phot. }\end{array}$ & $\begin{array}{l}\sigma_{T_{\text {eff }}} \\
\text { Phot. }\end{array}$ & $\begin{array}{l}\log g \\
\text { Spec. }\end{array}$ & $\begin{array}{l}\sigma_{\log g} \\
\text { Spec. }\end{array}$ & $\begin{array}{l}\log g \\
\text { Phot. }\end{array}$ & $\begin{array}{l}\sigma_{\log g} \\
\text { Phot. }\end{array}$ & $\begin{array}{c}v \sin i \\
{\left[\mathrm{~km} \mathrm{~s}^{-1}\right]}\end{array}$ & $\begin{array}{c}\sigma_{\mathrm{vsini}} \\
{\left[\mathrm{km} \mathrm{s}^{-1}\right]}\end{array}$ & binarity \\
\hline 139845816 & 8.02 & RS Gru & $7853^{12}$ & - & $6933^{7}$ & $81^{7}$ & $P$ & - & $3.53^{7}$ & - & $40^{15}$ & - & binary $^{24}$ \\
\hline 141026903 & 6.37 & HD 35184 & - & - & $8017^{8}$ & $206^{8}$ & - & - & $3.7^{5}$ & - & $94^{25}$ & $6^{25}$ & - \\
\hline 144309524 & 11.33 & TYC 8459-201-1 & - & - & $7818^{6}$ & $217^{6}$ & - & - & $4.11^{6}$ & $1.76^{6}$ & - & - & - \\
\hline 144387364 & 7.16 & BF Phe & - & - & $7241^{5}$ & $185^{5}$ & - & - & $4.02^{5}$ & $0.17^{14}$ & 80 & - & - \\
\hline 147085268 & 8.02 & HD 203880 & - & - & $7046^{26}$ & - & - & - & - & - & - & - & - \\
\hline 147113185 & 5.30 & HD 204018 & - & - & $7118^{2}$ & - & - & - & - & - & - & - & binary $^{27}$ \\
\hline 150101501 & 9.49 & HD 42780 & - & - & $8051^{28}$ & $200^{28}$ & - & - & - & - & - & - & - \\
\hline 150394126 & 6.54 & HD 46190 & $9790^{29}$ & $97^{29}$ & $8346^{5}$ & $669^{5}$ & - & - & $4.21^{5}$ & - & - & - & - \\
\hline 152864226 & 7.84 & HD 217417 & - & - & - & - & - & - & - & - & - & - & - \\
\hline 154842794 & 5.15 & $\pi \mathrm{PsA}$ & $7194^{18}$ & - & - & & 4.24 & - & - & - & - & & binary $^{30}$ \\
\hline 161172103 & 6.34 & CC Gru & - & - & $7031^{14}$ & $65^{14}$ & - & - & $3.55^{14}$ & $0.14^{9}$ & $122^{18}$ & - & - \\
\hline 166808854 & 4.88 & $\eta$ Hor & - & - & $7203^{31}$ & $90^{31}$ & - & - & - & - & $7^{31}$ & $2^{31}$ & binary $^{32}$ \\
\hline 167602316 & 3.04 & $\alpha \mathrm{Pic}$ & - & - & $7551^{33}$ & $35^{33}$ & - & - & - & - & $206^{8}$ & - & - \\
\hline 183532876 & 11.24 & CD-34 16262 & - & - & $6844^{6}$ & $203^{6}$ & - & - & $4.18^{6}$ & $1.49^{6}$ & - & - & - \\
\hline 183595451 & 5.73 & AI Scl & $7147^{39}$ & $145^{39}$ & $7502^{9}$ & $255^{9}$ & $3.21^{23}$ & - & & - & - & - & - \\
\hline 197686479 & 5.86 & BZ Gru & - & - & $6982^{14}$ & $81^{14}$ & - & - & $3.26^{14}$ & - & - & - & - \\
\hline 197759259 & 8.68 & HD 209689 & - & - & - & - & - & - & - & - & - & - & - \\
\hline 198035211 & 9.45 & HD 26058 & $7335^{1}$ & $442^{1}$ & $7950^{1}$ & $330^{1}$ & - & - & - & - & - & - & - \\
\hline 201250317 & 9.23 & HD 225032 & $7474^{1}$ & $83^{1}$ & $8115^{11}$ & - & $4.25^{1}$ & $0.16^{1}$ & - & - & - & - & - \\
\hline 206660904 & 7.65 & HD 216290 & - & - & - & - & - & - & - & - & - & - & - \\
\hline 211379298 & 9.46 & HD 203513 & - & - & $8019^{28}$ & $200^{28}$ & - & - & - & - & - & - & - \\
\hline 219332123 & 7.16 & DR Gru & - & - & $7447^{14}$ & $137^{14}$ & - & - & $3.82^{14}$ & $0.1^{34}$ & - & - & - \\
\hline 224280541 & 7.21 & HD 222701 & - & - & $7420^{2}$ & - & - & - & - & - & - & - & - \\
\hline 224285142 & 8.68 & HD 222987 & - & - & - & - & - & - & - & - & - & - & - \\
\hline 224285325 & 7.01 & SX Phe & $9138^{23}$ & - & - & - & $5.02^{23}$ & - & - & - & $18^{15}$ & - & - \\
\hline 229059574 & 6.15 & HD 210111 & $7400^{14}$ & $100^{14}$ & $7550^{14}$ & $123^{14}$ & $3.8^{14}$ & $0.1^{14}$ & $3.84^{14}$ & - & $30^{14}$ & - & binary $^{35}$ \\
\hline 229150702 & 5.76 & BD Phe & $7950^{39}$ & - & $8050^{9}$ & $274^{9}$ & 3.83 & - & $3.99^{9}$ & $0.09^{14}$ & $118^{31}$ & $15^{31}$ & - \\
\hline 229154157 & 9.53 & HD 11667 & $7061^{1}$ & $81^{1}$ & $7179^{4}$ & $146^{4}$ & $3.48^{1}$ & $0.12^{1}$ & - & - & $31^{1}$ & - & - \\
\hline 229810275 & 9.03 & HD 19098 & - & - & - & - & - & - & - & - & - & - & - \\
\hline 231014033 & 9.23 & HD 10961 & $7482^{1}$ & $83^{1}$ & $8069^{11}$ & - & $4.34^{1}$ & $0.16^{1}$ & - & - & $39^{1}$ & - & - \\
\hline 231020078 & 9.01 & HD 11333 & - & - & - & - & - & - & - & - & - & - & - \\
\hline 231048083 & 6.55 & FG Eri & $8986^{23}$ & $400^{23}$ & $7998^{8}$ & $93^{8}$ & $3.93^{23}$ & $0.5^{23}$ & $3.15^{5}$ & - & $107^{31}$ & $4^{31}$ & - \\
\hline 231632224 & 12.72 & 2MASS J21101928-5750453 & - & - & $7027^{6}$ & $202^{6}$ & - & - & $4.17^{6}$ & - & - & - & - \\
\hline 234498473 & 5.21 & HD 4125 & $7943^{39}$ & $92^{39}$ & - & - & - & - & - & - & $15^{31}$ & $5^{31}$ & binary ${ }^{32}$ \\
\hline 234516307 & 8.78 & HD 5648 & - & - & - & - & - & - & - & - & - & - & - \\
\hline 234528371 & 8.97 & HD 6622 & - & - & $7230^{36}$ & $27^{36}$ & - & - & - & - & - & - & - \\
\hline
\end{tabular}


Table A1: Stellar parameters for the TESS $\delta$ Sct and $\gamma$ Dor pulsators observed at 2-min cadence during the first two pointings (Sectors 1 and 2) in the ecliptic Southern hemisphere. The columns indicate: the TESS Input Catalogue (TIC) number, the TESS magnitude, an alternative identifier of the star, spectroscopic (Spec.) and photometric (Phot) effective temperatures and $\log g$ values and their associated uncertainties from literature respectively. The reference is indicated next to each value. In addition, we list the projected rotational velocity and binarity status as available in literature.

\begin{tabular}{|c|c|c|c|c|c|c|c|c|c|c|c|c|c|}
\hline TIC & $\begin{array}{l}\text { TESS } \\
\text { mag }\end{array}$ & $\begin{array}{c}\text { alternative } \\
\text { identifier }\end{array}$ & $\begin{array}{l}T_{\text {eff }}[K] \\
\text { Spec. }\end{array}$ & $\begin{array}{l}\sigma_{T_{\text {eff }}} \\
\text { Spec. }\end{array}$ & $\begin{array}{l}T_{\text {eff }}[K] \\
\text { Phot. }\end{array}$ & $\begin{array}{l}\sigma_{T_{\text {eff }}} \\
\text { Phot. }\end{array}$ & $\begin{array}{l}\log g \\
\text { Spec. }\end{array}$ & $\begin{array}{l}\sigma_{\log g} \\
\text { Spec. }\end{array}$ & $\begin{array}{l}\log g \\
\text { Phot. }\end{array}$ & $\begin{array}{l}\sigma_{\log g} \\
\text { Phot. }\end{array}$ & $\begin{array}{c}v \sin i \\
{\left[\mathrm{~km} \mathrm{~s}^{-1}\right]}\end{array}$ & $\begin{array}{c}\sigma_{\mathrm{vsini}} \\
{\left[\mathrm{km} \mathrm{s}^{-1}\right]}\end{array}$ & binarity \\
\hline 234548714 & 7.21 & BS Tuc & $7337^{37}$ & - & $7328^{14}$ & $102^{14}$ & - & - & $3.84^{14}$ & $0.17^{38}$ & $130^{15}$ & - & - \\
\hline 237318602 & 9.18 & HD 216941 & - & - & $7175^{4}$ & $496^{4}$ & - & - & - & - & - & - & - \\
\hline 237881239 & 9.38 & HD 8052 & $7477^{1}$ & $83^{1}$ & $7238^{4}$ & $469^{4}$ & $4.15^{1}$ & $0.16^{1}$ & - & - & $198^{1}$ & - & - \\
\hline 238185398 & 9.14 & HD 23537 & - & - & $8173^{11}$ & - & - & - & - & - & - & - & - \\
\hline 246845553 & 8.35 & HD 1619 & - & - & - & - & - & - & - & - & - & - & - \\
\hline 246853154 & 8.03 & HD 2026 & - & - & $6577^{2}$ & - & - & - & - & - & - & - & - \\
\hline 253917376 & 8.07 & UV PsA & $7918^{12}$ & - & $6889^{7}$ & $154^{7}$ & $4.00^{12}$ & - & - & - & - & - & - \\
\hline 260353074 & 8.96 & HD 44596 & - & - & $7031^{26}$ & - & - & - & - & - & - & - & - \\
\hline 260416268 & 5.47 & $v \mathrm{Pic}$ & $7537^{20}$ & $76^{20}$ & - & - & - & - & - & - & - & - & - \\
\hline 260654645 & 12.44 & 2MASS J06324522-5748198 & - & - & $6826^{6}$ & $201^{6}$ & - & - & $4.18^{6}$ & - & - & - & - \\
\hline 261089835 & 11.45 & TYC 9492-1017-1 & - & - & $6978^{6}$ & $205^{6}$ & - & - & $4.17^{6}$ & - & - & - & - \\
\hline 265566844 & 7.52 & BX Ind & $6968^{12}$ & - & $6762^{7}$ & $88^{7}$ & $3.62^{7}$ & - & $0.19^{7}$ & - & - & - & - \\
\hline 267094416 & 8.51 & HD 18006 & - & - & $7172^{2}$ & - & - & - & - & - & - & - & - \\
\hline 269994543 & 8.63 & HD 197648 & - & - & - & - & - & - & - & - & - & - & - \\
\hline 270067755 & 7.09 & 2MASS J20472005-2624547 & - & - & $7098^{5}$ & $163^{5}$ & - & - & $3.83^{5}$ & - & - & - & \\
\hline 277682809 & 8.32 & HD 208154 & - & - & - & - & - & - & - & - & - & - & - \\
\hline 278611926 & 7.51 & HD 210767 & - & - & - & - & - & - & - & - & - & - & binary $^{19}$ \\
\hline 279613634 & 8.77 & HD 54480 & - & - & $7147^{28}$ & $150^{28}$ & - & - & - & - & - & - & - \\
\hline 303584611 & 6.74 & BS Scl & $8216^{23}$ & $400^{23}$ & $7764^{5}$ & $175^{5}$ & $3.76^{23}$ & $0.5^{23}$ & $3.83^{5}$ & - & - & - & - \\
\hline 308396022 & 10.99 & TYC 8928-1300-1 & - & - & $6730^{5}$ & $248^{5}$ & - & - & $3.81^{5}$ & - & - & - & - \\
\hline 340705975 & 6.51 & AW Scl & - & - & $8103^{5}$ & $143^{5}$ & - & - & $3.60^{5}$ & - & - & - & binary $^{30}$ \\
\hline 348762920 & 5.42 & HD 20313 & - & - & $7000^{2}$ & - & - & - & 3.98 & - & $75^{15}$ & - & binary ${ }^{16}$ \\
\hline 348772511 & 7.25 & CP Oct & $7028^{39}$ & $120^{39}$ & $6900^{38}$ & $100^{38}$ & $3.44^{23}$ & - & $3.63^{38}$ & - & $72^{40}$ & - & binary $^{41}$ \\
\hline 350431472 & 9.57 & HD 38081 & - & - & $7624^{28}$ & $75^{28}$ & - & - & - & - & - & - & - \\
\hline 350563225 & 9.07 & HD 39131 & $7632^{42}$ & $95^{42}$ & $7827^{11}$ & - & $4.1^{42}$ & $0.18^{42}$ & - & - & $157^{42}$ & - & - \\
\hline 355547586 & 12.56 & 2MASS J21242025-5751539 & - & - & $7073^{6}$ & $206^{6}$ & - & - & $4.16^{6}$ & - & - & - & - \\
\hline 355687188 & 9.72 & HD 224852 & $6834^{43}$ & $300^{43}$ & $6865^{5}$ & $360^{5}$ & $3.50^{43}$ & $0.3^{43}$ & $3.55^{5}$ & - & - & - & - \\
\hline 358070081 & 9.47 & HD 16938 & $7090^{44}$ & - & $7032^{4}$ & $208^{4}$ & $3.95^{44}$ & - & - & - & $109^{1}$ & - & - \\
\hline 358502706 & 11.60 & 2MASS J04032009-8341586 & - & - & $7369^{6}$ & $210^{6}$ & - & - & - & - & - & - & - \\
\hline 364399376 & 7.16 & V393 Car & - & - & $7400^{34}$ & $100^{34}$ & - & - & $3.7^{34}$ & - & - & - & - \\
\hline 381204458 & 8.76 & HD 19532 & - & - & - & - & - & - & - & - & - & - & - \\
\hline 381857833 & 8.15 & HD 204352 & - & - & $7308^{45}$ & $81^{45}$ & - & - & - & - & - & - & - \\
\hline 382551468 & 10.44 & CD-53 251 & $6761^{46}$ & $93^{46}$ & $5836^{6}$ & $190^{6}$ & $4.03^{46}$ & $0.10^{46}$ & $3.64^{6}$ & - & $17^{46}$ & $1^{46}$ & - \\
\hline 394015973 & 7.96 & BE Ind & $7830^{23}$ & - & - & - & $3.56^{23}$ & - & - & - & $39^{23}$ & $1^{23}$ & - \\
\hline 396720223 & 9.57 & HD 28001 & $8720^{52}$ & - & $7233^{4}$ & $186^{4}$ & - & - & - & - & - & - & - \\
\hline
\end{tabular}


Table A1: Stellar parameters for the TESS $\delta$ Sct and $\gamma$ Dor pulsators observed at 2-min cadence during the first two pointings (Sectors 1 and 2) in the ecliptic Southern hemisphere. The columns indicate: the TESS Input Catalogue (TIC) number, the TESS magnitude, an alternative identifier of the star, spectroscopic (Spec.) and photometric (Phot) effective temperatures and $\log g$ values and their associated uncertainties from literature respectively. The reference is indicated next to each value.

In addition, we list the projected rotational velocity and binarity status as available in literature.

\begin{tabular}{|c|c|c|c|c|c|c|c|c|c|c|c|c|c|}
\hline TIC & $\begin{array}{c}\text { TESS } \\
\text { mag }\end{array}$ & $\begin{array}{l}\text { alternative } \\
\text { identifier }\end{array}$ & $\begin{array}{l}T_{\text {eff }}[K] \\
\text { Spec. }\end{array}$ & $\begin{array}{l}\sigma_{T_{\text {eff }}} \\
\text { Spec. }\end{array}$ & $\begin{array}{l}T_{\text {eff }}[K] \\
\text { Phot. }\end{array}$ & $\begin{array}{l}\sigma_{T_{\text {eff }}} \\
\text { Phot. }\end{array}$ & $\begin{array}{l}\log g \\
\text { Spec. }\end{array}$ & $\begin{array}{l}\sigma_{\log g} \\
\text { Spec. }\end{array}$ & $\begin{array}{l}\log g \\
\text { Phot. }\end{array}$ & $\begin{array}{l}\sigma_{\log g} \\
\text { Phot. }\end{array}$ & $\begin{array}{c}v \sin i \\
{\left[\mathrm{~km} \mathrm{~s}^{-1}\right]}\end{array}$ & $\begin{array}{c}\sigma_{\mathrm{vsini}} \\
{\left[\mathrm{km} \mathrm{s}^{-1}\right]}\end{array}$ & binarity \\
\hline 399572664 & 9.78 & HD 215353 & - & - & $7826^{5}$ & $247^{5}$ & - & 1 & $4.10^{5}$ & $1.56^{6}$ & - & - & - \\
\hline 402047030 & 8.05 & HD 221098 & - & - & - & - & - & - & - & - & - & - & - \\
\hline 402318229 & 8.96 & HD 221576 & - & - & - & - & - & - & - & - & - & - & - \\
\hline 426012953 & 9.62 & HD 8096 & $8000^{1}$ & $83^{1}$ & $8919^{11}$ & - & $4.50^{1}$ & $0.16^{1}$ & - & - & - & - & - \\
\hline 431589510 & 11.81 & TYC 9158-919-1 & $7023^{47}$ & $41^{47}$ & - & - & $4.04^{47}$ & $0.17^{47}$ & - & - & - & - & - \\
\hline 439399707 & 8.82 & HD 225186 & - & - & $7518^{2}$ & $377^{2}$ & - & 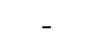 & - & - & - & - & - \\
\hline 441110063 & 5.54 & $8 \mathrm{PsA}$ & - & - & - & - & - & - & - & - & $23^{25}$ & - & - \\
\hline 469844770 & 9.62 & HD 199247 & - & - & $7950^{5}$ & $208^{5}$ & - & - & $4.30^{5}$ & - & - & - & - \\
\hline 469933721 & 5.88 & DQ Gru & - & - & - & - & - & - & - & - & $179^{25}$ & - & - \\
\hline
\end{tabular}


${ }^{1}$ Kunder et al. (2017) ${ }^{2}$ McDonald et al. (2012) ${ }^{3}$ Huber et al. (2016) ${ }^{4}$ Stevens et al. (2017) ${ }^{5}$ McDonald et al. (2017) ${ }^{6}$ Stassun et al. $(2018)^{7}$ Casagrande et al. $(2011)^{8}$ Zorec \& Royer (2012b) ${ }^{9}$ David \& Hillenbrand (2015b) ${ }^{10}$ Díaz et al. (2011) ${ }^{11}$ Ammons et al. (2006) ${ }^{12}$ Rainer et al. (2017) ${ }^{13}$ Renson et al. (1991) ${ }^{14}$ Paunzen et al. (2002) ${ }^{15}$ Rodríguez \& López-González (2000) ${ }^{16}$ Liakos \& Niarchos (2017) ${ }^{17}$ Bailer-Jones (2011) ${ }^{18}$ Erspamer \& North (2003) ${ }^{19}$ Tokovinin \& Kiyaeva (2016) ${ }^{20}$ Soubiran et al. (2010) ${ }^{21}$ Eggleton \& Tokovinin (2008) ${ }^{22}$ Uesugi \& Fukuda (1970) ${ }^{23}$ Rainer et al. (2016) ${ }^{24}$ Liakos \& Niarchos (2016) ${ }^{25}$ Royer et al. (2002) ${ }^{26}$ Smalley et al. (2011) ${ }^{27}$ Corbally (1984) ${ }^{28}$ Gaia Collaboration (2018) ${ }^{29}$ Sloan et al. (2004) ${ }^{30}$ Pourbaix et al. (2004b) ${ }^{31}$ Ammler-von Eiff \& Reiners (2012) ${ }^{32}$ Malkov et al. (2012) ${ }^{33}$ Royer et al. (2007) ${ }^{34}$ Helt (1984) ${ }^{35}$ Faraggiana et al. (2004) ${ }^{36}$ Munari et al. (2014) ${ }^{37}$ Cheng et al. $(2017)^{38}$ Niemczura et al. (2017) ${ }^{39}$ Soubiran et al. (2016) ${ }^{40}$ González et al. (2008) ${ }^{41}$ Paparo et al. (1996) ${ }^{42}$ Kordopatis et al. (2013) ${ }^{43}$ Zwitter et al. (2010) $\quad 44$ Siebert et al. (2011) $\quad 45$ Masana et al. (2006) $\quad 46$ Zwintz et al. (2014) $\quad 47$ Martell et al. (2017) 
Table A2: Stellar parameters and variability type of the 2-min cadence TESS $\delta$ Scuti and $\gamma$ Dor pulsators determined in this work. In this table we list the TESS Input Catalogue (TIC) name, an alternative name for each star, the effective temperatures derived using the SED method (Section 4.1), the luminosities derived based on the Gaia and when available Hipparcos parallaxes and the respective uncertainties (Section 4.3). In column 9 we describe the variability type determined from the TESS data. The question mark means that the variability type is uncertain (e.g. due to unresolved peaks). HAGD denotes a High Amplitude $\gamma$ Dor star. The last column describes the chemical peculiarity as described in Renson \& Manfroid (2009).

\begin{tabular}{|c|c|c|c|c|c|c|c|c|c|}
\hline TIC & $\begin{array}{l}\text { alternative } \\
\text { identifier }\end{array}$ & $\begin{array}{c}T_{\text {eff }}[\mathrm{K}] \\
\text { SED }\end{array}$ & $\sigma T_{\text {eff }}$ & $\begin{array}{c}\log \left(\mathrm{L} / \mathrm{L}_{\odot}\right) \\
\text { Gaia }\end{array}$ & $\sigma \log \left(\mathrm{L} / \mathrm{L}_{\odot}\right)$ & $\begin{array}{c}\log \left(\mathrm{L} / \mathrm{L}_{\odot}\right) \\
\text { HIPP. }\end{array}$ & $\sigma \log \left(\mathrm{L} / \mathrm{L}_{\odot}\right)$ & $\begin{array}{c}\text { variability } \\
\text { type }\end{array}$ & $\begin{array}{l}\text { chem. } \\
\text { pec. }\end{array}$ \\
\hline 9632550 & BS Aqr & 6760 & 140 & 1.62 & 0.03 & 1.96 & 0.94 & HADS & \\
\hline 12470841 & HD 923 & 8090 & 230 & 1.64 & 0.02 & 2.01 & 0.43 & one large peak only & \\
\hline 12784216 & HD 213125 & 6390 & 120 & 1.14 & 0.01 & & & $\delta$ Sct/hybrid? & \\
\hline 12974182 & HD 218003 & 7670 & 170 & 1.103 & 0.008 & 1.08 & 0.04 & rot?/binary? & \\
\hline 29281339 & HD 198501 & 7430 & 160 & 1.205 & 0.007 & 1.17 & 0.04 & almost const & \\
\hline 30531417 & XX Dor & 6800 & 150 & 1.64 & 0.03 & & & HAGD & \\
\hline 32176627 & HD 209970 & 7140 & 140 & 0.883 & 0.006 & 0.92 & 0.04 & const & \\
\hline 32197339 & HD 210139 & 8120 & 180 & 1.9 & 0.1 & 1.69 & 0.13 & $\gamma$ Dor (unresolved) & \\
\hline 33911462 & HD 222828 & 6700 & 140 & 1.28 & 0.02 & 1.52 & 0.33 & $\delta$ Sct & \\
\hline 33984043 & HD 223466 & 8340 & 80 & 1.257 & 0.008 & 1.33 & 0.05 & const & $\mathrm{Am}$ \\
\hline 34038105 & HD 223991A & 8640 & 90 & 1.131 & 0.007 & 1.02 & 0.06 & const & \\
\hline 38587180 & TX Ret & 7230 & 160 & 1.343 & 0.006 & 1.38 & 0.1 & $\delta$ Sct & $\mathrm{Am}$ \\
\hline 38602305 & $\theta$ Ret $\mathrm{A}$ & 12000 & 390 & 2.101 & 0.006 & 2.09 & 0.04 & binary or multiple system & \\
\hline 38847248 & $\theta$ Tuc & 7510 & 150 & 1.831 & 0.007 & 1.77 & 0.03 & $\delta$ Sct $/ \mathrm{EB}$ & \\
\hline 44627561 & HD 215559 & 6410 & 140 & 1.44 & 0.02 & & & $\delta$ Sct/binary?/Ap? & $\mathrm{Am}$ \\
\hline 49677785 & HD 220687 & 7360 & 180 & 1.42 & 0.02 & & & $\delta$ Sct / binary & \\
\hline 51991595 & 2MASS J01011055-6843069 & 6890 & 150 & 1.25 & 0.03 & & & HADS & $\mathrm{Am}$ \\
\hline 52244754 & HD 8438 & 6650 & 140 & 1.240 & 0.009 & & & $\delta$ Sct & $\mathrm{Am}$ \\
\hline 52258534 & BG Hyi & 6990 & 140 & 1.233 & 0.006 & 1.33 & 0.1 & $\delta$ Sct & \\
\hline 66434034 & HD 5497 & 7830 & 200 & 0.94 & 0.01 & & & $\delta$ Sct & \\
\hline 70744515 & HD 225077 & 9870 & 550 & 1.27 & 0.03 & 1.21 & 0.18 & const & \\
\hline 71334169 & HD 209430 & 7150 & 160 & 1.21 & 0.01 & 0.62 & 0.21 & $\delta$ Sct & \\
\hline 79394646 & $\theta$ Ind $\mathrm{A}$ & 8090 & 60 & 1.074 & 0.008 & 1.1 & 0.01 & $\delta$ Sct/binary/rot & Am \\
\hline 80474886 & AZ Phe & 7030 & 120 & 1.308 & 0.006 & 1.39 & 0.09 & $\delta$ Sct & $\mathrm{Am}$ \\
\hline 88277481 & HD 210740 & 7520 & 170 & 0.852 & 0.008 & & & $\gamma$ Dor/hybrid/1 peak only in $\delta$ Sct regime & \\
\hline 89464315 & WX PsA & 7250 & 150 & 1.68 & 0.01 & 1.7 & 0.02 & $\delta$ Sct & $\mathrm{Am}$ \\
\hline 89542582 & HD 223587 & 7430 & 170 & 1.09 & 0.03 & 0.81 & 0.36 & $\delta$ Sct & \\
\hline 92734713 & HD 200835 & 8420 & 270 & 1.12 & 0.01 & & & $\delta$ Sct & \\
\hline 99839685 & HD 204972 & 7210 & 160 & 1.10 & 0.01 & 0.84 & 0.16 & $\delta$ Sct/ hybrid? & \\
\hline 102090493 & HD 7454 & 5890 & 110 & 1.28 & 0.01 & 0.79 & 0.21 & $\delta$ Sct /Ap/binary? & \\
\hline 116157537 & BB Phe & 7070 & 120 & 1.749 & 0.006 & 1.7 & 0.05 & $\delta$ Sct & \\
\hline 126659093 & ZZ Mic & 8110 & 210 & 1.2 & 0.02 & 1.03 & 0.33 & HADS & \\
\hline 137796620 & HD 216728 & 7840 & 200 & 0.89 & 0.03 & & & $\delta$ Sct & \\
\hline 139131968 & $\tau^{3} \mathrm{Gru}$ & 7550 & 160 & 1.457 & 0.009 & 1.38 & 0.02 & const & $\mathrm{Am}$ \\
\hline 139232635 & $\theta$ Gru & 6990 & 90 & 1.4 & 0.02 & 1.45 & 0.02 & rot & \\
\hline
\end{tabular}


Table A2: Stellar parameters and variability type of the 2-min cadence TESS $\delta$ Scuti and $\gamma$ Dor pulsators determined in this work. In this table we list the TESS Input Catalogue (TIC) name, an alternative name for each star, the effective temperatures derived using the SED method (Section 4.1), the luminosities derived based on the Gaia and when available Hipparcos parallaxes and the respective uncertainties (Section 4.3). In column 9 we describe the variability type determined from the TESS data. The question mark means that the variability type is uncertain (e.g. due to unresolved peaks). HAGD denotes a High Amplitude $\gamma$ Dor star. The last column describes the chemical peculiarity as described in Renson \& Manfroid (2009).

\begin{tabular}{|c|c|c|c|c|c|c|c|c|c|}
\hline TIC & $\begin{array}{l}\text { alternative } \\
\text { identifier }\end{array}$ & $\begin{array}{c}T_{\text {eff }}[\mathrm{K}] \\
\mathrm{SED}\end{array}$ & $\sigma T_{\text {eff }}$ & $\begin{array}{c}\log \left(\mathrm{L} / \mathrm{L}_{\odot}\right) \\
\text { Gaia }\end{array}$ & $\sigma \log \left(\mathrm{L} / \mathrm{L}_{\odot}\right)$ & $\begin{array}{c}\log \left(\mathrm{L} / \mathrm{L}_{\odot}\right) \\
\text { HIPP. }\end{array}$ & $\sigma \log \left(\mathrm{L} / \mathrm{L}_{\odot}\right)$ & $\begin{array}{c}\text { variability } \\
\text { type }\end{array}$ & $\begin{array}{l}\text { chem. } \\
\text { pec. }\end{array}$ \\
\hline 139825582 & CF Ind & 7250 & 140 & 1.504 & 0.009 & 1.47 & 0.14 & $\delta$ Sct/ hybrid? & $\mathrm{Am}$ \\
\hline 139845816 & RS Gru & 7780 & 170 & 1.45 & 0.01 & 1.41 & 0.25 & HADS & \\
\hline 141026903 & HD 35184 & 8120 & 160 & 1.455 & 0.005 & 1.43 & 0.03 & const & \\
\hline 144309524 & TYC 8459-201-1 & 7220 & 240 & 1.32 & 0.04 & & & HADS & \\
\hline 144387364 & BF Phe & 7350 & 170 & 1.013 & 0.005 & 0.97 & 0.06 & $\delta$ Sct/ hybrid? & \\
\hline 147085268 & HD 203880 & 7290 & 160 & 1.062 & 0.008 & & & $\delta$ Sct/ hybrid? & \\
\hline 147113185 & HD 204018 & 6900 & 120 & 1.175 & 0.006 & 1.21 & 0.03 & const & $\mathrm{Am}$ \\
\hline 150101501 & HD 42780 & 7920 & 240 & 1.09 & 0.01 & & & $\delta$ Sct & \\
\hline 150394126 & HD 46190 & 8850 & 100 & 1.167 & 0.005 & 1.16 & 0.02 & $\delta$ Sct & \\
\hline 152864226 & HD 217417 & 7200 & 150 & 0.93 & 0.01 & 0.91 & 0.1 & $\delta$ Sct/ hybrid & \\
\hline 154842794 & $\pi \mathrm{PsA}$ & 7420 & 110 & 0.783 & 0.007 & 0.82 & 0.02 & $\gamma$ Dor & $\mathrm{Am}$ \\
\hline 161172103 & CC Gru & 7160 & 150 & 1.466 & 0.006 & 1.57 & 0.06 & $\delta$ Sct & \\
\hline 166808854 & $\eta$ Hor & 7840 & 500 & 1.06 & 0.02 & 1.14 & 0.04 & $\delta$ Sct & $\mathrm{Am}$ \\
\hline 167602316 & $\alpha \mathrm{Pic}$ & 7350 & 660 & & & 1.56 & 0.05 & $\delta$ Sct/hybrid & \\
\hline 183532876 & CD-34 16262 & 7140 & 280 & 1.55 & 0.04 & & & HADS & $\mathrm{Am}$ \\
\hline 183595451 & AI Scl & 7360 & 140 & 1.234 & 0.009 & 1.23 & 0.02 & $\delta$ Sct & $\mathrm{Am}$ \\
\hline 197686479 & BZ Gru & 6950 & 130 & 1.69 & 0.01 & 1.77 & 0.05 & $\delta$ Sct & \\
\hline 197759259 & HD 209689 & 7510 & 180 & 1.135 & 0.009 & & & $\delta$ Sct/ hybrid & \\
\hline 198035211 & HD 26058 & 7530 & 170 & 0.95 & 0.01 & & & $\delta$ Sct & \\
\hline 201250317 & HD 225032 & 7410 & 250 & 1.01 & 0.01 & & & $\delta$ Sct & $\mathrm{Am}$ \\
\hline 206660904 & HD 216290 & 7450 & 170 & 1.33 & 0.01 & 1.26 & 0.12 & const & \\
\hline 211379298 & HD 203513 & 8110 & 210 & 1.20 & 0.03 & & & $\delta$ Sct /binary & $\mathrm{Am}$ \\
\hline 219332123 & DR Gru & 7830 & 160 & 1.062 & 0.007 & 1.02 & 0.07 & $\delta$ Sct/ hybrid? & \\
\hline 224280541 & HD 222701 & 7420 & 140 & 1.35 & 0.01 & 1.46 & 0.08 & const & \\
\hline 224285142 & HD 222987 & 7730 & 190 & 1.23 & 0.01 & 1.09 & 0.18 & $\delta$ Sct & \\
\hline 224285325 & SX Phe & 7320 & 170 & 0.844 & 0.009 & 0.79 & 0.04 & HADS & $\mathrm{Am}$ \\
\hline 229059574 & HD 210111 & 7910 & 110 & 1.157 & 0.006 & 1.15 & 0.03 & $\delta$ Sct & \\
\hline 229150702 & BD Phe & 8300 & 80 & 1.343 & 0.005 & 1.33 & 0.02 & $\delta$ Sct & $\mathrm{Am}$ \\
\hline 229154157 & HD 11667 & 6660 & 140 & 1.33 & 0.01 & & & $\delta$ Sct & \\
\hline 229810275 & HD 19098 & 8250 & 220 & 1.17 & 0.008 & & & const & \\
\hline 231014033 & HD 10961 & 7150 & 150 & 0.88 & 0.01 & & & $\delta$ Sct & \\
\hline 231020078 & HD 11333 & 7450 & 180 & 0.94 & 0.02 & & & $\delta$ Sct/ hybrid? & \\
\hline 231048083 & FG Eri & 7940 & 140 & 1.984 & 0.007 & 2 & 0.07 & developed $\delta$ Sct? & \\
\hline 231632224 & 2MASS J21101928-5750453 & 7210 & 280 & 1.56 & 0.08 & & & HADS & \\
\hline 234498473 & HD 4125 & 7960 & 160 & 1.61 & 0.08 & & & $\delta$ Sct & $\mathrm{Am}$ \\
\hline 234516307 & HD 5648 & 7520 & 180 & 1.097 & 0.008 & & & $\delta$ Sct & \\
\hline
\end{tabular}


Table A2: Stellar parameters and variability type of the 2-min cadence TESS $\delta$ Scuti and $\gamma$ Dor pulsators determined in this work. In this table we list the TESS Input Catalogue (TIC) name, an alternative name for each star, the effective temperatures derived using the SED method (Section 4.1), the luminosities derived based on the Gaia and when available Hipparcos parallaxes and the respective uncertainties (Section 4.3). In column 9 we describe the variability type determined from the TESS data. The question mark means that the variability type is uncertain (e.g. due to unresolved peaks). HAGD denotes a High Amplitude $\gamma$ Dor star. The last column describes the chemical peculiarity as described in Renson \& Manfroid (2009).

\begin{tabular}{|c|c|c|c|c|c|c|c|c|c|}
\hline TIC & $\begin{array}{c}\text { alternative } \\
\text { identifier }\end{array}$ & $\begin{array}{c}T_{\text {eff }}[\mathrm{K}] \\
\text { SED }\end{array}$ & $\sigma T_{\text {eff }}$ & $\begin{array}{c}\log \left(\mathrm{L} / \mathrm{L}_{\odot}\right) \\
\text { Gaia }\end{array}$ & $\sigma \log \left(\mathrm{L} / \mathrm{L}_{\odot}\right)$ & $\begin{array}{c}\log \left(\mathrm{L} / \mathrm{L}_{\odot}\right) \\
\text { HIPP. }\end{array}$ & $\sigma \log \left(\mathrm{L} / \mathrm{L}_{\odot}\right)$ & $\begin{array}{c}\text { variability } \\
\text { type }\end{array}$ & $\begin{array}{l}\text { chem. } \\
\text { pec. }\end{array}$ \\
\hline 234528371 & HD 6622 & 7180 & 220 & 0.91 & 0.007 & & & $\delta$ Sct & $\mathrm{Am}$ \\
\hline 234548714 & BS Tuc & 7290 & 160 & 0.981 & 0.005 & 0.95 & 0.04 & $\delta$ Sct/ hybrid? & \\
\hline 237318602 & HD 216941 & & & & & & & $\delta$ Sct & Am \\
\hline 237881239 & HD 8052 & 7400 & 180 & 0.913 & 0.008 & & & $\delta$ Sct/ hybrid? & \\
\hline 238185398 & HD 23537 & 8100 & 210 & 1.011 & 0.007 & & & roAp candidate? & \\
\hline 246845553 & HD 1619 & 7200 & 160 & 1.00 & 0.01 & & & spots & Am \\
\hline 246853154 & HD 2026 & 8340 & 160 & 1.39 & 0.01 & 1.27 & 0.12 & rot?/binary? & \\
\hline 253917376 & UV PsA & 6850 & 140 & 1.24 & 0.01 & & & $\delta$ Sct & $\mathrm{Am}$ \\
\hline 260353074 & HD 44596 & 7010 & 150 & 1.26 & 0.02 & & & $\delta$ Sct/ hybrid? & \\
\hline 260416268 & $v \mathrm{Pic}$ & 7830 & 300 & 1.20 & 0.02 & 1.04 & 0.02 & rot?/binary? & $\mathrm{Am}$ \\
\hline 260654645 & 2MASS J06324522-5748198 & 7050 & 160 & 1.24 & 0.03 & & & HADS & \\
\hline 261089835 & TYC 9492-1017-1 & 6310 & 160 & 1.32 & 0.03 & & & HADS & $\mathrm{Am}$ \\
\hline 265566844 & BX Ind & 6640 & 130 & 1.46 & 0.01 & 1.09 & 0.1 & $\delta$ Sct? 1 peak & \\
\hline 267094416 & HD 18006 & 7300 & 160 & 1.08 & 0.02 & 1 & 0.1 & almost const & \\
\hline 269994543 & HD 197648 & 7430 & 170 & 0.95 & 0.02 & & & $\delta$ Sct/ hybrid? & Am \\
\hline 270067755 & 2MASS J20472005-2624547 & 7790 & 200 & 1.077 & 0.008 & 1.13 & 0.1 & almost const & $\mathrm{Am}$ \\
\hline 277682809 & HD 208154 & 8320 & 240 & 0.936 & 0.007 & 0.98 & 0.14 & $\delta$ Sct/ hybrid? & Am \\
\hline 278611926 & HD 210767 & 7370 & 140 & 4 & 3 & & & rot?/binary? & \\
\hline 279361762 & V383 Car & 6950 & 140 & 1.612 & 0.008 & 1.38 & 0.1 & $\delta$ Sct & \\
\hline 279613634 & HD 54480 & 7190 & 160 & 1.00 & 0.01 & & & $\delta$ Sct & \\
\hline 303584611 & BS Scl & 8050 & 140 & 1.370 & 0.008 & 1.27 & 0.04 & $\delta$ Sct & \\
\hline 308396022 & TYC 8928-1300-1 & 6860 & 150 & 0.94 & 0.01 & & & $\delta$ Sct/HADS/hybrid? & \\
\hline 340705975 & AW Scl & 8010 & 150 & 1.757 & 0.009 & 1.63 & 0.06 & Ap? & \\
\hline 348762920 & HD 20313 & 7370 & 130 & 1.461 & 0.007 & 1.48 & 0.04 & $\delta$ Sct/hybrid & \\
\hline 348772511 & CP Oct & 6810 & 140 & 1.47 & 0.01 & 1.63 & 0.12 & $\delta$ Sct & \\
\hline 350431472 & HD 38081 & 7020 & 170 & 0.998 & 0.007 & & & $\delta$ Sct & \\
\hline 350563225 & HD 39131 & 7610 & 180 & 1.07 & 0.01 & & & $\delta$ Sct & \\
\hline 355547586 & 2MASS J21242025-5751539 & 7240 & 170 & 1.05 & 0.08 & & & HADS & \\
\hline 355687188 & HD 224852 & 6510 & 140 & 1.45 & 0.02 & & & HADS & \\
\hline 358070081 & HD 16938 & 6540 & 140 & 0.9 & 0.2 & & & $\delta$ Sct & \\
\hline 358502706 & TYC 9492-02623-1 & 7220 & 190 & 1.12 & 0.02 & & & HADS & \\
\hline 364399376 & V393 Car & 7330 & 170 & 1.489 & 0.008 & & & $\delta$ Sct & \\
\hline 381204458 & HD 19532 & 7730 & 180 & 1.48 & 0.01 & & & $\delta$ Sct / binary & $\mathrm{Am}$ \\
\hline 381857833 & HD 204352 & 7460 & 170 & 1.102 & 0.008 & 0.98 & 0.11 & $\delta$ Sct & \\
\hline 382551468 & CD-53 251 & 6080 & 110 & 0.83 & 0.01 & & & const & \\
\hline 394015973 & BE Ind & 7520 & 180 & 1.33 & 0.01 & 1.42 & 0.23 & $\delta$ Sct/ hybrid? & \\
\hline
\end{tabular}


Table A2: Stellar parameters and variability type of the 2-min cadence TESS $\delta$ Scuti and $\gamma$ Dor pulsators determined in this work. In this table we list the TESS Input Catalogue (TIC) name, an alternative name for each star, the effective temperatures derived using the SED method (Section 4.1), the luminosities derived based on the Gaia and when available

Hipparcos parallaxes and the respective uncertainties (Section 4.3). In column 9 we describe the variability type determined

from the TESS data. The question mark means that the variability type is uncertain (e.g. due to unresolved peaks). HAGD denotes a High Amplitude $\gamma$ Dor star. The last column describes the chemical peculiarity as described in Renson \& Manfroid (2009).

\begin{tabular}{|c|c|c|c|c|c|c|c|c|c|}
\hline TIC & $\begin{array}{c}\text { alternative } \\
\text { identifier }\end{array}$ & $\begin{array}{l}T_{\text {eff }}[\mathrm{K}] \\
\text { SED }\end{array}$ & $\sigma T_{\mathrm{eff}}$ & $\begin{array}{c}\log \left(\mathrm{L} / \mathrm{L}_{\odot}\right) \\
\text { Gaia }\end{array}$ & $\sigma \log \left(\mathrm{L} / \mathrm{L}_{\odot}\right)$ & $\begin{array}{l}\log \left(\mathrm{L} / \mathrm{L}_{\odot}\right) \\
\text { HIPP. }\end{array}$ & $\sigma \log \left(\mathrm{L} / \mathrm{L}_{\odot}\right)$ & $\begin{array}{c}\text { variability } \\
\text { type }\end{array}$ & $\begin{array}{l}\text { chem. } \\
\text { pec. }\end{array}$ \\
\hline 396720223 & HD 28001 & 7200 & 160 & 1.13 & 0.02 & & & $\delta$ Sct/ hybrid? & \\
\hline 399572664 & HD 215353 & 8050 & 210 & 1.26 & 0.02 & & & $\delta$ Sct / binary?/rot? & \\
\hline 402047030 & HD 221098 & 7080 & 150 & 1.213 & 0.008 & 1.25 & 0.16 & const & \\
\hline 402318229 & HD 221576 & 7650 & 180 & 1.07 & 0.02 & & & $\delta$ Sct/ hybrid? & $\mathrm{Am}$ \\
\hline 426012953 & HD 8096 & 8360 & 290 & 1.38 & 0.02 & & & const & \\
\hline 431589510 & TYC 9158-919-1 & 6930 & 190 & 1.2 & 0.3 & & & HADS & \\
\hline 439399707 & HD 225186 & 7250 & 170 & 0.99 & 0.02 & 1.19 & 0.25 & $\delta$ Sct/binary/flares & \\
\hline 441110063 & $8 \mathrm{PsA}$ & 7720 & 160 & 1.122 & 0.008 & 1.17 & 0.02 & $\delta$ Sct & \\
\hline 469844770 & HD 199247 & 7980 & 210 & 0.92 & 0.01 & & & $\delta$ Sct & \\
\hline 469933721 & $\mathrm{~V}^{*} \mathrm{DQ} \mathrm{Gru}$ & 0 & 0 & 1.691 & 0.008 & 1.63 & 0.07 & $\gamma$ Dor $/ \delta$ Sct/hybrid? & \\
\hline
\end{tabular}




\section{APPENDIX B: AUTHOR AFFILIATIONS}

${ }^{1}$ Stellar Astrophysics Centre, Ny Munkegade 120, 8000, Aarhus, Denmark

${ }^{2}$ Instituto de Astrofísica e Ciências do Espaço, Universidade do Porto, CAUP, Rua das Estrelas, PT4150-762 Porto, Portugal

${ }^{3}$ Institute of Astronomy, KU Leuven, Celestijnenlaan 200D, 3001 Leuven, Belgium

${ }^{4}$ Sydney Institute for Astronomy (SIfA), School of Physics, University of Sydney, NSW 2006, Australia

${ }^{5}$ Jeremiah Horrocks Institute, University of Central Lancashire, Preston PR1 2HE, UK

${ }^{6}$ LESIA, Observatoire de Paris, Université PSL, CNRS, Sorbonne Université, Université de Paris, 5 place Jules Janssen, 92195 Meudon, France

${ }^{7}$ Instytut Astronomiczny, Uniwersytet Wrocławski, Kopernika 11, 51-622 Wrocław, Poland

${ }^{8}$ Instituto de Astronomía, Universidad Nacional Autónoma de México, Ap. P. 877, Ensenada, BC 22860, Mexico

${ }^{9}$ Dept. Física Teórica y del Cosmos, Universidad de Granada, Campus de Fuentenueva s/n, E-18071 Granada, Spain

${ }^{10}$ Instituto de Astrofísica de Andalucía, Glorieta de la Astronomía s/n, Granada, Spain

${ }^{11}$ Department of Physics, Institute for Advanced Studies in Basic Sciences (IASBS), Zanjan 45137-66731, Iran

${ }^{12}$ Department of Physics, Faculty of Science, University of Zanjan P.O. Box 45195-313, Zanjan, Iran

${ }^{13}$ Nicolaus Copernicus Astronomical Center, Bartycka 18, PL-00-716 Warsaw, Poland

${ }^{14}$ Çanakkale Onsekiz Mart University, Faculty of Sciences and Arts, Physics Department, 17100 Çanakkale, Turkey

${ }^{15}$ Center for Astrophysics | Harvard \& Smithsonian, 60 Garden Street, Cambridge, MA 02138, USA

${ }^{16}$ Department of Physics and Astronomy, University of British Columbia, Vancouver, Canada

${ }^{17}$ Department of Theoretical Physics and Astrophysics, Masaryk University, Kotlarska 2, 61137 Brno, Czech Republic

18 Instituto de Astrofísica La Plata, CONICET-UNLP, Paseo del Bosque s/n, 1900 La Plata, Argentina

${ }^{19}$ Department of Physics, Lehigh University, 16 Memorial Drive East, Bethlehem, PA, 18015, USA

${ }^{20}$ School of Earth and Space Exploration, Arizona State University, Tempe, AZ 85281, USA

${ }^{21}$ Department of Physics and Kavli Institute for Astrophysics and Space Research, Massachusetts Institute of Technology, Cambridge, MA 02139, USA

${ }^{22}$ Department of Earth, Atmospheric and Planetary Sciences, Massachusetts Institute of Technology, Cambridge, MA 02139, USA

${ }^{23}$ Department of Aeronautics and Astronautics, MIT, 77 Massachusetts Avenue, Cambridge, MA 02139, USA

${ }^{24}$ Astrophysics Group, Keele University, Staffordshire, ST5 5BG, United Kingdom

${ }^{25}$ Department of Physics, University of York, Heslington, York, YO10 5DD, UK

${ }^{26}$ Konkoly Observatory, MTA CSFK, H-1121, Konkoly Thege Miklós út 15-17, Budapest, Hungary

${ }^{27} 22$ MTA CSFK Lendület Near-Field Cosmology Research Group
${ }^{28}$ Dept. Física Teórica y del Cosmos, Universidad de Granada, Campus de Fuentenueva s/n, E-18071, Granada, Spain

${ }^{29}$ Instituto de Astrofísica de Andalucía, Glorieta de la Astronomía s/n, Granada, Spain

${ }^{30}$ Yunnan Observatories, Chinese Academy of Sciences, 396 Yangfangwang, Guandu District, Kunming, 650216, P. R. China

${ }^{31}$ Key Laboratory for the Structure and Evolution of Celestial Objects, Chinese Academy of Sciences, 396 Yangfangwang, Guandu District, Kunming, 650216, P. R. China ${ }^{32}$ Center for Astronomical Mega-Science, Chinese Academy of Sciences, 20A Datun Road, Chaoyang District, Beijing, 100012, P. R. China

${ }^{33}$ Universität Innsbruck, Institut für Astro- und Teilchenphysik, Technikerstrasse 25, A-6020 Innsbruck, Austria

${ }^{34}$ Dpto. de Astrofísica, Centro de Astrobiología (CSICINTA), ESAC, Camino Bajo del Castillo s/n, 28692, Spain

${ }^{35}$ Department of Chemistry and Physics, Florida Gulf Coast University, 10501 FGCU Blvd., Fort Myers, FL 33965 USA

${ }^{36}$ Royal Observatory of Belgium, Ringlaan 3, 1180, Brussel, Belgium

${ }^{37}$ Center for Exoplanets and Habitable Worlds, 525 Davey Laboratory, The Pennsylvania State University, University Park, PA 16802, USA

${ }^{38}$ Los Alamos National Laboratory, MS T-082, Los Alamos, NM 87545 USA

${ }^{39}$ Physics Department, Bloomsburg University, 200 E 2nd St, Bloomsburg, PA 17815, USA

${ }^{40}$ Physics Department, Mount Allison University, Sackville, NB, E4L 1E6, Canada

${ }^{41}$ Astrophysics Research Group, Faculty of Engineering and Physical Sciences, University of Surrey, Guildford GU2 $7 \mathrm{XH}, \mathrm{UK}$

${ }^{42}$ National Astronomical Research Institue of Thailand, 260 Moo 4, T. Donkaew, A. Maerim, Chiangmai, 50180 Thailand

${ }^{43}$ Departamento de Física e Astronomia, Faculdade de Ciências da Universidade do Porto, Portugal

${ }^{44}$ Department of Physics \& Astronomy, Camosun College, Victoria, BC, Canada.

${ }^{45}$ IRAP, Université de Toulouse, CNRS, UPS, CNES, 14, avenue Edouard Belin, F-31400 Toulouse, France

${ }^{46}$ Department of Theoretical Physics and Astrophysics, Masaryk University, Kotlářská 2, 61137 Brno, Czech Republic

${ }^{47}$ Astronomical Institute, Czech Academy of Sciences, Fričova 298, 25165, Ondřejov, Czech Republic

${ }^{48}$ Institute of Astronomy and NAO, Bulgarian Academy of Sciences, blvd.Tsarigradsko chaussee 72, Sofia 1784, Bulgaria

${ }^{49}$ Institute for Astrophysics, University of Vienna, Türkenschanzstrasse 17, 1180 Vienna, Austria

This paper has been typeset from a $\mathrm{T}_{\mathrm{E}} \mathrm{X} / \mathrm{LAT}_{\mathrm{E}} \mathrm{X}$ file prepared by the author. 